Aus dem Institut für Diagnostik und Interventionelle Neuroradiologie

(PD. Dr. med. M. N. Psychogios)

der Medizinischen Fakultät der Universität Göttingen

\title{
Wie exakt sind die Größenbestimmungen von intrakraniellen Aneurysmen? \\ -Eine multimodale Phantomstudie-
}

\author{
INAUGURAL-DISSERTATION \\ zur Erlangung des Doktorgrades \\ der Medizinischen Fakultät der \\ Georg-August-Universität zu Göttingen
}

vorgelegt von

Thilo Khakzad

aus

Berlin

Göttingen 2019 
Dekan:

Referent/in

Ko-Referent/in:

Drittreferent/in:
Prof. Dr. Wolfgang Brück

PD. Dr. med. M. N. Psychogios

Prof. Dr. med. Dorothee Mielke

Datum der mündlichen Prüfung: 14.07 .2020 
Hiermit erkläre ich, die Dissertation mit dem Titel " Wie exakt sind die Größenbestimmungen von intrakraniellen Aneurysmen? -Eine multimodale Phantomstudie-" eigenständig angefertigt und keine anderen als die von mir angegebenen Quellen und Hilfsmittel verwendet zu haben.

Göttingen, den 29.05.2019 


\section{Inhaltsverzeichnis}

Abkürzungsverzeichnis.................................................................................... III

Abbildungsverzeichnis ..................................................................................IV

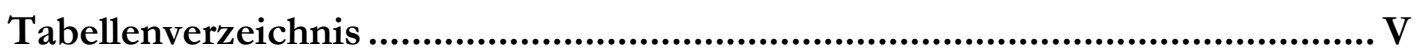

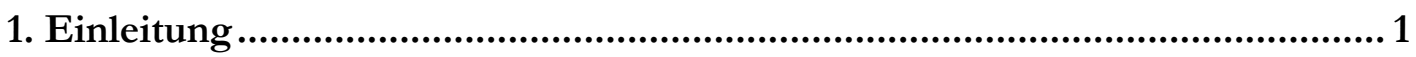

2. Therotische Fundierung ........................................................................... 3

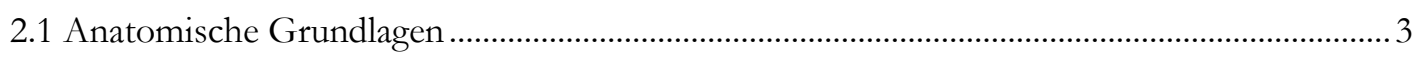

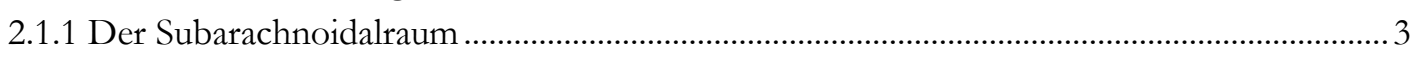

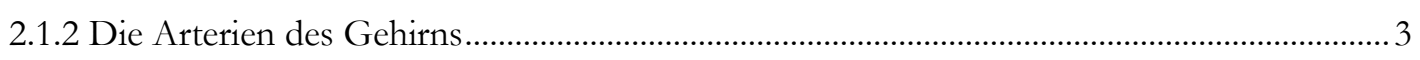

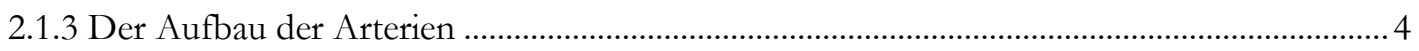

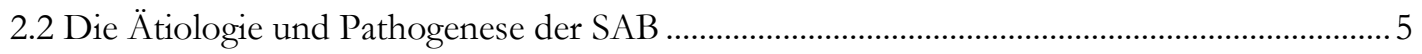

2.2.1 Das Aneurysma, Einteilung und Definition ........................................................................ 6

2.2.2 Die Entstehung von zerebralen Aneurysmen .................................................................

2.2.2.1 Hämodynamischer Stress als Ursache der Aneurysmaformation .......................................10

2.2.2.2 Genetische Aspekte der Aneurysmabildung ....................................................................11

2.2.2.3 Das Rauchen als Risikofaktor für die Entstehung eines Aneurysmas................................12

2.2.3 Das Rupturrisiko bei vorhandenem zerebralem Aneurysma ...............................................12

2.2.4 Pathophysiologie der aneurysmatischen SAB ...................................................................16

2.3 Akutsymptome und klinische Zeichen der SAB ……………………………………….......19

2.4 Präklinisches und klinisches Vorgehen bei Akutsymptomen der SAB ...................................19

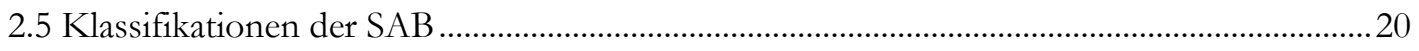

2.6 Apparative und interventionelle Diagnostik ...........................................................................22

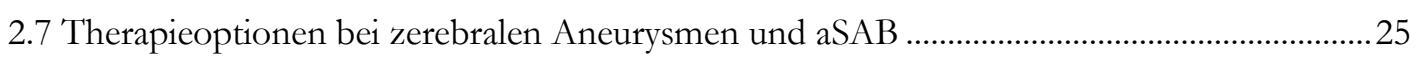

2.7.1 Die endovaskulären Behandlungsmöglichkeiten intrakranieller Aneurysmen....................25

2.7.2 Die Neurochirurgische Behandlungsmethode, das Clipping...............................................27

2.7.3 Die ISAT-Studie, ein Paradigmenwechsel in der Behandlung der aSAB.............................28

2.8 Das Management der SAB und ihre Komplikationen ................................................................ 31

2.9 Die Computertomographie .................................................................................................. 32

2.10 Die Flachbilddetektor-Computertomographie (FDCT) …………………………………....... 33

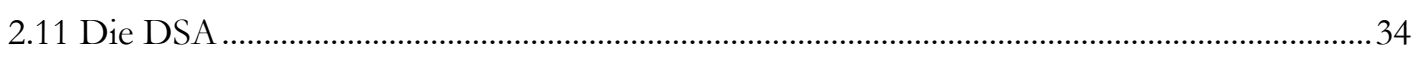

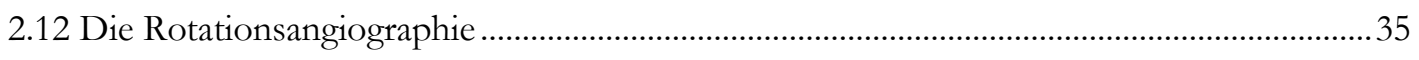

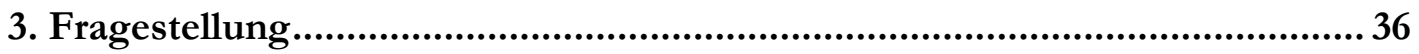

3.1 Vergleich der Messgenauigkeit der vier verschiedenen bildgebenden Verfahren (FDCTA, DSA, MDCTA und Rotationsangiographie) zu den bekannten Referenzgrößen der

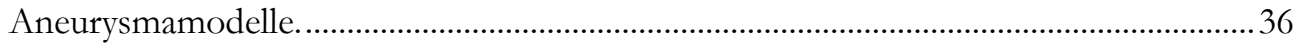

3.2 Vergleich der Messgenauigkeit zwischen dem aus der Studie hervorgegangenen Goldstandard und den anderen bildgebenden Verfahren. ...................................................36 
3.3 Die Rolle der Erfahrung der Untersucher bei der Ausmessung und Nutzung der verschieden bildgebenden Verfahren.

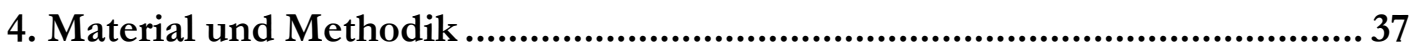

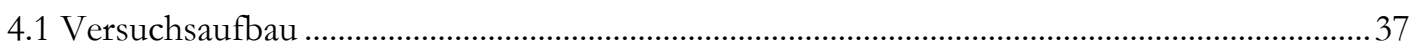

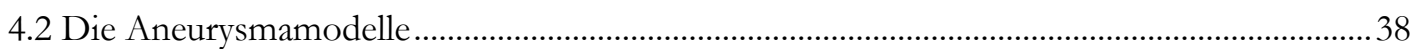

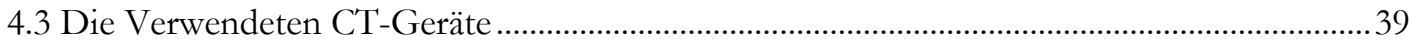

4.4 Verwendete Abkürzungen und untersuchte Verfahren und Rekonstruktionen im

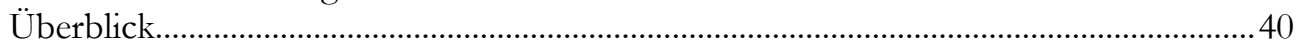

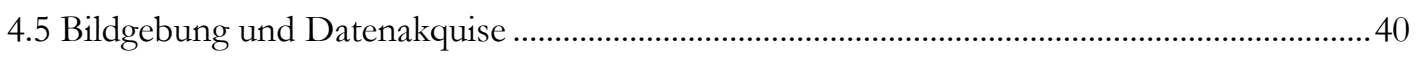

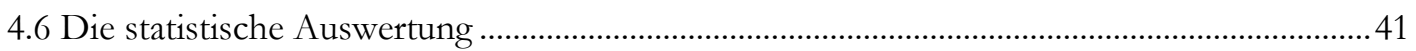

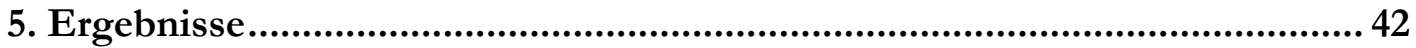

5.1 Graphische Darstellung der Messergebnisse .............................................................................4

5.2 Tabellarische Darstellung der Messergebnisse .........................................................................50

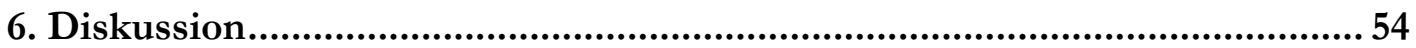

6.1 Hierarchisierung der Verfahren und Auswertung der Ergebnisse ..........................................54

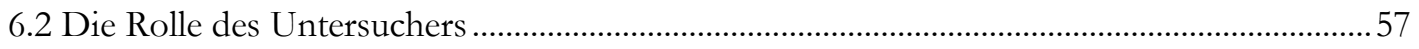

6.3 Die Gesundheitsrisiken der untersuchten Verfahren..............................................................57

6.4 Wissenschaftliche Einordnung...........................................................................................

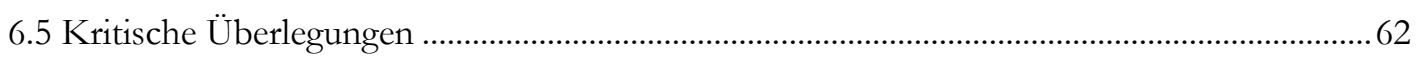

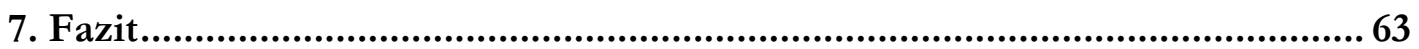

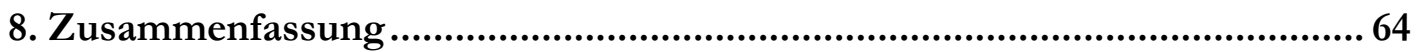

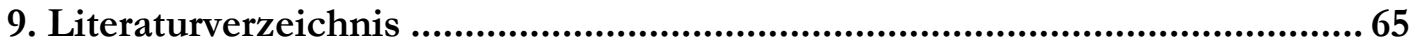

Aus der Arbeit hervorgegangene Veröffentlichung.......................................... 74

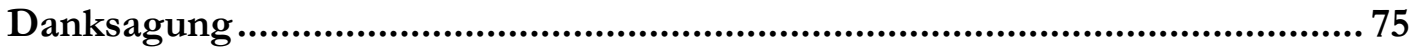

Lebenslauf......................................................Fehler! Textmarke nicht definiert. 


\section{Abkürzungsverzeichnis}

A - Arterie

Aa - Arteriea

ACA - Arteria cerebri anterior

ACI - Arteria carotis interna

ACM - Arteria cerebri media

aSAB - aneurysmatische Subarachnoidalblutung

CT - Computertomographie

EZM - Extrazellulärmatrix

FD-CTA - Flachdetektor-Computertomographie-Angiographie

MCP-1 - monocyte chemoattractant protein-1

MD-CTA - Multidetektor-Computertomographie-Angiographie

MMP -Matrix-Metalloproteasen

MPR - multiplanare Rekonstruktion

NF-KB -nuclear factor, kappa-light-chain-enhancer' of activated B-cells

SAB - Subarachnoidalblutung

VRT - volumen-renderin-technique

WFNS - World Federation of Neurosurgery

ZNS - Zentrales Nervensystem

2D-DSA - zweidimensionale (konventionelle) digitale Subtraktionsangiographie

3D-DSA - Rotationsangiographie/ dreidimensionale digitale Subtraktionsangiographie 


\section{Abbildungsverzeichnis}

Abbildung 1: Circulus arteriosus cerebri.

Abbildung 2: Verschiedene Aneurysmaformen

Abbildung 3: Foto des Versuchaufbaus; Das Aneurysmamodell in einer menschlichen Schädelkalotte auf dem Angiographietisch des biplane Artis Q

Abbildung 4: Graphische Darstellung in Bland-Altman-Plots der DSA_rotation_near gegen die FDCTA

Abbildung 5: Graphische Darstellung in Bland-Altman-Plots der DSA_rotation_near gegen die FDCTA_VRT

Abbildung 6: Graphische Darstellung in Bland-Altman-Plots der DSA_rotation_near gegen die

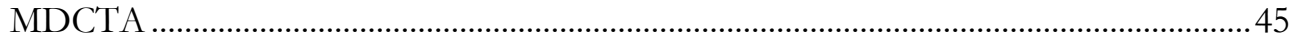

Abbildung 7: Graphische Darstellung in Bland-Altman-Plots der DSA_rotation_near gegen die MDCTA_VRT

Abbildung 8: Graphische Darstellung in Bland-Altman-Plots der DSA_rotation_near gegen die DSA_long.

Abbildung 9: Graphische Darstellung in Bland-Altman-Plots der DSA_rotation_near gegen die DSA_middle ................................................................................................................ 46

Abbildung 10: Graphische Darstellung in Bland-Altman-Plots der DSA_rotation_near gegen die

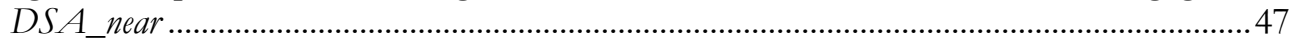

Abbildung 11: Graphische Darstellung in Bland-Altman-Plots der DSA_rotation_near gegen die $D S A \_$rotation_long...

Abbildung 12: Graphische Darstellung in Bland-Altman-Plots der DSA_rotation_near gegen die DSA_rotation_middle .......................................................................................................4

Abbildung 13: Graphische Darstellung in Bland-Altman-Plots der DSA_rotation_near gegen die Rotation_VRT...

Abbildung 14: Graphische Darstellung in Bland-Altman-Plots der DSA_rotation_near gegen die Rotation_MPR 


\section{Tabellenverzeichnis}

Tabelle 1: Pathomechanismus und die resultierenden Komplikationen der SAB; modifiziert nach Masuhr et al. 2013.

Tabelle 2: SAB nach 5 klinischen Schweregraden eingeordnet; modifiziert nach Hunt und Hess 1968

Tabelle 3: Glascow Coma Scale; modifiziert nach Teasdale und Jennett 1974 _..........................21

Tabelle 4: WFNS-Score, modifiziert nach Teasdale et al. 1988 ....................................................22

Tabelle 5: Fisher-Klassifikation zur qualitativen Einschätzung der SAB in der Comptertomographie; modifiziert nach Fisher et al. 1980 .............................................23

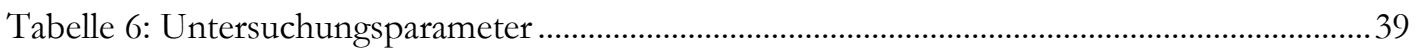

Tabelle 7: Aneurysma I. mittlere Abweichung zu den Referenzgrößen (alle drei Dimensionen

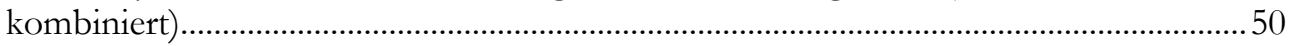

Tabelle 8: Aneurysma II. mittlere Abweichung zu den Referenzgrößen (alle drei Dimensionen

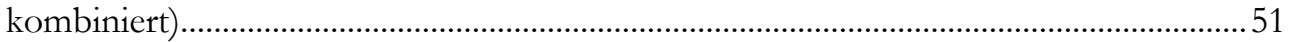

Tabelle 9: Statistische Analyse der Bland-Altman Plots im Vergleich der DSA_rotation_near mit

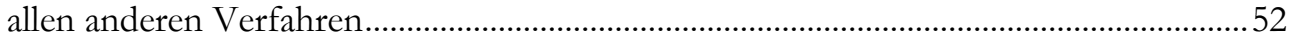

Tabelle 10: Interclass correlation coefficient für alle untersuchten Techniken zum Vergleich der Untersucher-Reliabilität 53 


\section{Einleitung}

Die Subarachnoidalblutung $(\mathrm{SAB})$ ist eine potenziell tödliche Erkrankung aus dem Formenkreis der Apoplexien, bei der Blut durch ein geplatztes Gefäß in den Subarachnoidalraum gelangt und durch starke, plötzlich auftretende Kopfschmerzen gekennzeichnet ist. Häufig geht die SAB mit fokal-neurologischen Defiziten bis hin zur Bewusstlosigkeit einher (Hop et al. 1997; Linn et al. 1998).

International liegt die Prävalenz statistisch bei ca. 6-10 auf 100.000 Einwohner, wobei sich deutliche regionale Unterschiede feststellen lassen. Auch wenn die SAB lediglich 15\% aller Apoplexe ausmacht, so hat sie doch häufig fatale Folgen für den einzelnen $\mathrm{Pa}$ tienten (Sudlow et al. 1997). Mit einer Letalitätsrate von 51\% stellt die primäre SAB einen schweren medizinischen Notfall dar, der mit einem Altersgipfel von ca. 50 Jahren, relativ junge Patienten betrifft. 10\% der Betroffenen sterben vor dem Eintreffen medizinischer Hilfe, weitere 25\% innerhalb des ersten Monats. Daraus ergibt sich eine Letalität von 35\% innerhalb des ersten Monats (Hop et al. 1997). Ein weiteres Drittel verbleibt lebenslang pflegebedürftig und selbst $46 \%$ der Patienten mit gutem Outcome klagen über kognitiv-neurologische Defizite (Mayer et al. 2002). Die beschriebene Prognose der SAB bleibt daher, trotz der sich immer verbessernden medizinischen Versorgung, schlecht. Hoffnung geben Metaanalysen wie die von Nieuwkamp et. al., die einen stetig positiven Trend hinsichtlich der Prognose verzeichnen. Die Verbesserung sei in der Einführung von Managementstrategien begründet und lässt sich mit einer Sterberatereduktion von 17\% zwischen 1973 und 2002, trotz steigendem mittleren Erkrankungsalter, quantifizieren (Nieuwkamp et al. 2009). Der größte Risikofaktor für ein schlechtes Outcome ist die Schwere der SAB. Rosengart et. al. verglichen vier Studien aus den neunziger Jahren mit insgesamt 3567 Patienten und kamen zu dem Ergebnis, dass die posthämorrhagischen Infarkte und der Aufnahmestatus, gemessen am WFNS-Score, das prognosebestimmende Element bei Patienten mit akuter SAB sind (Rosengart et al. 2007). Die Verhinderung posthämorrhagischer Infarkte ist daher eines der primären Therapieziele und wird durch eine schnellstmögliche Okklusion der Blutungsquelle und der weitmöglichsten Verhinderung entstehender intrakranieller Vasospasmen erreicht. Die Blutmenge im Subarachnoidalraum korreliert mit dem Auftreten von Vasospasmen und dem daraus resultierenden poststenotischen Ischämien (Fisher et al. 1980). Heutzutage gilt die en- 
dovaskuläre Therapie als die Therapie der ersten Wahl und ermöglicht eine schnelle endosakuläre Okklusion und zwar meist über die Einführung von Platinspiralen, den sog. Coils, in den Aneurysmasack.

Die permanente technologische Weiterentwicklung ermöglicht heutzutage eine schnelle Diagnostik und eine interventionelle Therapie intrakranieller Aneurysmen. Aufgrund der wachsenden Zahlen zerebraler Bildgebungen finden sich immer häufiger inzidentelle Aneurysmen, d. h. zufällig entdeckte symptomlose Aneurysmen. Die Okklusion eines inzidentellen Aneurysmas geht häufig mit Risiken einher, die zahlenmäßig, je nach Aneurysmaeigenschaft, nahe dem Rupturrisiko des Aneurysmas oder höher liegen. In diesen Fällen fehlt die Indikation zur Therapie. Genau diese Prävention und Versorgung zufällig gefundener Aneurysmen ist jedoch der hauptsächliche Ruptur-vorbeugende Therapieansatz, neben der medikamentösen Optimierung und Anpassungen des Lebensstils zur Reduktion des kardiovaskulären Risikos. Die Reduktion des Behandlungsrisikos, der Morbidität und die Verbesserung des Therapieerfolgs bei inzidentellen Aneurysmen sollten daher im Zentrum zukünftiger Forschung stehen. Diese experimentelle Arbeit setzt sich zum Ziel, das beste Verfahren zu explorieren, mit dem es möglich ist, im Kontext klinischer Praktikabilität die exakten Größenverhältnisse von intrakraniellen Aneurysmen zu bestimmen. Weiterhin wurden nicht nur die bildgebenden Verfahren als solche, sondern auch deren Anwendbarkeit untersucht, wobei auch Bezug auf die klinische Erfahrung des messenden Neuroradiologen gelegt wird. Die exakte Darstellung des Aneurysmas, seiner geometrischen Größenverhältnisse und Form, der zu- und abführenden Gefäße im dreidimensionalem Raum sind von entscheidender therapeutischer Brisanz. Dieses gilt nicht nur in Bezug auf die Wahl des Verfahrens, sondern auch auf dessen Planung und Wahl der zu nutzenden Medizinprodukte wie z.B. des Coils. Diese Forschungsarbeit verfolgt daher das Ziel, zu einer Verbesserung der diagnostischen und therapeutischen Versorgung von Patienten sowohl mit rupturierten als auch mit unrupturierten intrakraniellen Aneurysmen, beizutragen. 


\section{Therotische Fundierung}

\subsection{Anatomische Grundlagen}

Der Abschnitt Anatomische Grundlagen ist keine detaillierte Darstellung der Anatomie des zentralen Nervensystems (ZNS), sondern eine kleine Übersicht für den Leser, um das Folgende anatomisch einordnen zu können.

\subsubsection{Der Subarachnoidalraum}

Der Subarachnoidalraum (Syn. Spatium subarachnoidale) ist ein mit Liquor cerebrospinalis gefüllter Raum im ZNS. Die Pia Mater, auch weiche Hirnhaut genannt, liegt dem Parenchym ZNS direkt auf und folgt seiner Struktur bis in die Sulci und Gyri, das Windungsrelief des Gehirns. Die Arachnoidea, die sog. Spinnenhaut, bildet die äußere Grenze des Subarachnoidalraums und ist namensgebend. Sie folgt nicht den einzelnen Windungen des Gehirns, sondern stülpt sich über die beiden Hemisphären und vereinigt sich tief im Interhemisphärenspalt. Der Subarachnoidalraum mit seinem Liquor cerebrospinalis fungiert unter anderem als Polsterung für die weichen Strukturen des ZNS und wird auch als äußerer Liquorraum bezeichnet. Der Subarachnoidalraum besteht somit aus den kortikalen Sulci, den Zisternen (Erweiterungen des Subarachnoidalraums im Bereich der Schädelbasis) und dem spinalen Subarachnoidalraum. Neben dem Liquor befinden sich auch die Hirn- und Rückenmark versorgenden Arterien und Venen im Subarachnoidalraum.

\subsubsection{Die Arterien des Gehirns}

Die hirnversorgenden Arterien kann man in ein vorderes und hinteres Stromgebiet unterteilen. Das vordere Stromgebiet entstammt der paarig angelegten Arteria carotis Interna (ACI). Die ACI lässt sich in sieben Segmente unterteilen (C1-C7). Intrakraniell teilt sie sich in die Arteria cerebri media (ACM) und die Arteria cerebri anterior (ACA). Erstere ist wiederum in vier (M1-M4) Segmente unterteilt, wohingegen sich die ACA aus drei Segmenten zusammensetzt (A1-A3). Ihre Teilungsstelle wird Carotis-T genannt. Die Aa. vertebralis (V0-V4) bilden den Ausgangspunkt des hinteren Stromgebietes und vereinigen sich intrakraniell zur Arteria (A.) basilaris. Die Schädelbasisarterien bilden eine ringförmige Gefäßanastomose, den Circulus arteriosus Willisi (siehe Abbildung1). Dadurch verbinden sie nicht nur das vordere und hintere Stromgebiet, sondern auch die 
Versorgung beider Hemisphären miteinander. Auf alle Abgangsarterien und ihre Versorgungsgebiete wird nicht eingegangen, wenn es nicht in anderen Abschnitten erwähnt wird.

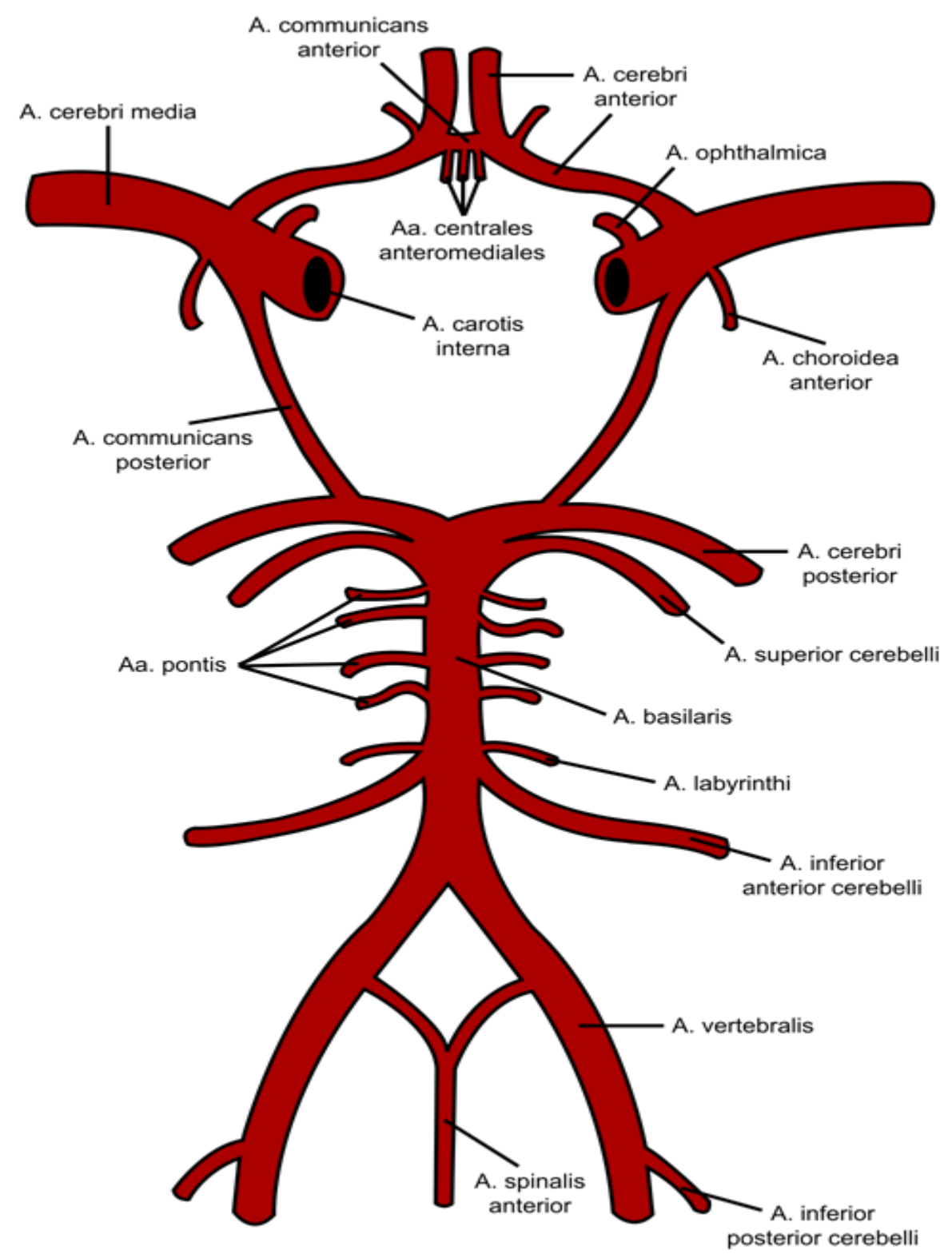

Abbildung 1: Circulus arteriosus cerebri

\subsubsection{Der Aufbau der Arterien}

Alle Blutgefäße, seien es Arterien oder Venen, bestehen aus Hauptkomponenten. Das Endothel, die entscheidende Grenze zwischen Intra- und Extravasalraum, kleidet die Arterien von innen aus. Es besteht aus einem einschichtigen Plattenepithel. Funktionell hat es mehrere Aufgaben. Es fungiert als Diffusionsbarriere und reguliert den Durchtritt von 
Mikro- und Makromolekülen in die subendotheliale Schicht. Je nach physiologischer Situation bestimmt das Endothel die Adhäsionseigenschaften der Gefäßinnenwand und ist damit ein wichtiger Regulator der Leukozytenemigration. Auch die Regulation der Blutgerinnung und der Gefäßweite ist Aufgabe des Endothels, welche durch die Expression und Sekretion von Oberflächenproteinen realisiert wird. Die glatte Muskulatur dient dem Erhalt der Wandspannung und ist somit unter anderem für die Einstellung der Gefäßweite mitverantwortlich. Die Beschaffenheit und Mechanik der Gefäßwand werden auch durch die Extrazellulärmatrix (EZM) bestimmt. Sie besteht aus Proteoglykanen, kollagenen und elastischen Fasern.

Arterien haben einen dreischichtigen Wandaufbau. Die Tunica intima, bestehend aus Endothel und der subendothelialen Schicht, ist die innere Auskleidung des Gefäßes und trennt sich von der Tunica media meist durch eine Membrana elastica interna, eine elastische Lamelle. Die Tunica media ist die Muskelschicht des Gefäßes. Sie beherbergt die glatten Muskelfasern sowie die EZM. In welchem Verhältnis Muskelzellen zu elastischen Fasern der EZM in der Tunica media vorhanden sind, hängt von der Herznähe des Gefäßes ab. Es werden Arterien vom elastischen Typ, sprich große herznahe Gefäße, zu deren Aufgaben der Erhalt der Windkesselfunktion gehört und Arterien vom muskulären Typ unterschieden. Auch die Tunica media ist, vor allem bei großen Gefäßen, durch eine elastische Lamelle, der Membrana elastica externa, von der ihr außen aufliegenden Schicht abzugrenzen. Die äußere Schicht der Gefäße nennt man Tunica adventitia oder externa. Als Bindegewebsschicht sorgt sie für die Befestigung des Gefäßes an seine Umgebung. In ihr befinden sich sowohl die postganglionären Axone des Sympathikus zur efferenten Innervation als auch die Vasa vasorum, die, das Blutgefäß versorgenden, Blutgefäße. Diese Vasa vasorum übernehmen grob gesagt die Versorgung der äußeren Hälfte der Gefäßwand. Die Tunica intima und die innere Schicht der Tunica media werden durch das Lumen der Arterien mit Sauerstoff versorgt.

\subsection{Die Ätiologie und Pathogenese der SAB}

Die SAB ereignet sich in 75-85\% aufgrund eines intrakraniellen Aneurysmas der hirnversorgenden Gefäße (Gijn und Rinkel 2001). Andere Ursachen der nicht-traumatisch bedingten SAB sind die AV-Malformationen von Gehirn und Rückenmark, Aneurysmen arteriosklerotischer Ätiologie, Blutungen von Tumoren, Sinusthrombosen, mykotische Aneurysmen, Vaskulitiden und Gerinnungsstörungen (Vlak et al. 2011). 
Da diese Dissertation sich mit der Ausmessung von Aneurysmen befasst und Aneurysmen ätiologisch den absolut größten Anteil aller SAB ausmachen, wird im Folgenden nur auf den Pathomechanismus der aSAB eingegangen.

\subsubsection{Das Aneurysma, Einteilung und Definition}

Der Begriff Aneurysma kommt aus dem Altgriechischen und bedeutet „Aufweitung“. In der Humanmedizin meint man damit die Aufweitung eines arteriellen Gefäßes. Bei einem Aneurysma ist die Gefäßwand stets pathologisch verändert und birgt daher ein erhöhtes Risiko zu reißen. Man spricht dann von einem rupturierten Aneurysma. Aneurysmen müssen von sog. Ektasien differenziert werden. Gefäßektasien sind permanente „Aufweitungen“ des Gefäßdurchmessers bei intakten Gefäßwandschichten. Um diesen Unterschied deutlich zu machen, werden Aneurysmen besser als Gefäßaussackungen übersetzt. Sie lassen sich in drei Unterformen einteilen, welche nachfolgend dargestellt werden.

Das „Aneurysma verum“ (echtes Aneurysma) ist eine sack- oder spindelförmige Erweiterung aller drei Gefäßwandschichten und der entscheidende Subtyp zerebraler Aneurysmen. Beim „Aneurysma dissecans“, dem dissezierenden Aneurysma, wühlt sich Blut zwischen die Wandschichten der Arterien und bildet dort eine zweite Blutbahn innerhalb des Gefäßes. Diese Nebenstrombahn kann die Gefäßwände so spalten, dass es zur Blockierung des eigentlichen Lumens kommt. Das „Aneurysma falsum oder spurium“ (falsches Aneurysma) beschreibt eine Hämatomentwicklung um eine von außerhalb des Gefäßes entstandene Verletzung der Gefäßwand. Das Hämatom drückt die verletzte Stelle kurzzeitig zu. Da der Blutaustritt durch das umliegende Gewebe begrenzt wird, kann es eine größere Blutung häufig herauszögern. Es besteht aber weiterhin ein erhöhtes Rupturrisiko. Durch die Entstehung von außen ist es definitionsgemäß kein echtes Aneurysma. Daher wählt man häufig die Bezeichnung „,falsches Aneurysma“.

Abbildung zwei soll das Beschriebene verdeutlichen. 


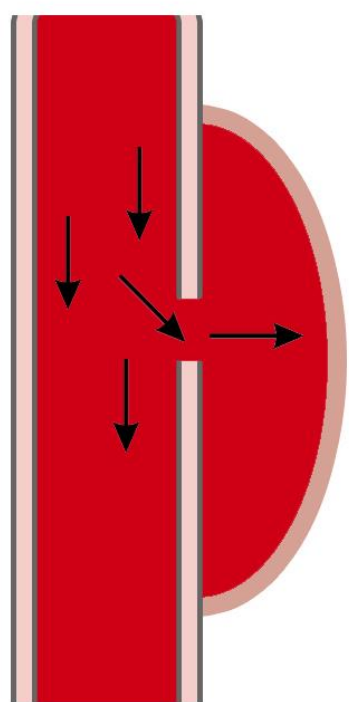

Aneurysma spurium

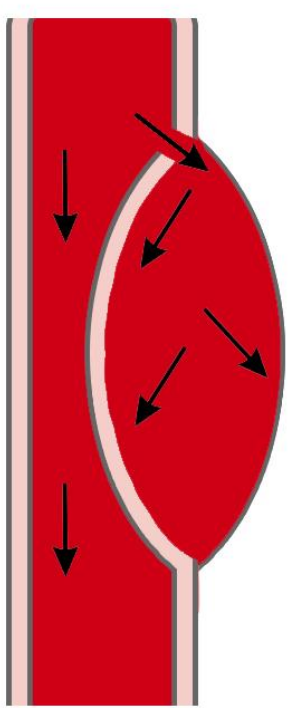

Aneurysma dissecans

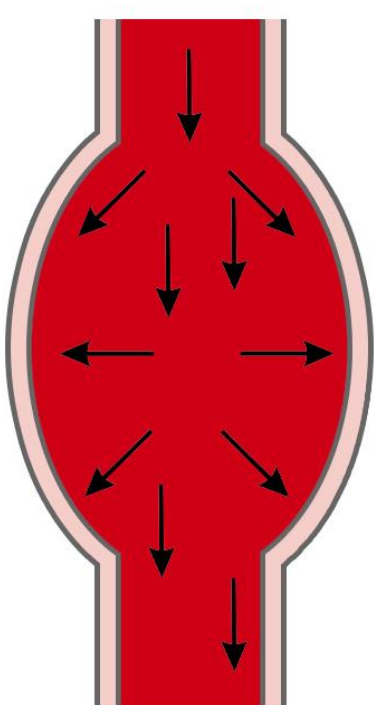

Aneurysma verum

Abbildung 2: Verschiedene Aneurysmaformen

\subsubsection{Die Entstehung von zerebralen Aneurysmen}

Der Pathomechanismus, der für die Entstehung von Aneurysmen verantwortlich ist, ist heutzutage noch immer nicht allumfassend geklärt. Verschiedene Modalitäten, wie z.B. entzündliche Prozesse der Gefäßwand, Lebensstilaspekte wie das Rauchen, aber auch genetische Prädispositionen, sind derzeit Gegenstand wissenschaftlicher Forschung. Durch Kenntnis der Entstehung, der Risikofaktoren oder des Rupturrisikos erhofft man sich Fortschritte in Prävention und Therapie. Wissenschaftlich erwiesen erscheint die Annahme, dass es zur Formation eines zerebralen Aneurysmas durch entzündliche Prozesse der arteriellen Gefäßwand kommt. Hämodynamischer Stress, der eine Dysfunktion des Endothels bewirkt, führt zu einem inflammatorischen Prozess. Eine Entzündung ist eine komplexe lokale oder systemische Reaktion des Körpers auf endo- oder exogene Reize. Das Immunsystem reagiert charakteristisch mit der Produktion und Rekrutierung von Antikörpern, Immunzellen und Entzündungsmediatoren. Dies geschieht zum Beispiel durch den Transkriptionsfaktor (nuclear factor, kappa-light-chain-enhancer' of activated Bcells) NF-KB. NF-KB wird durch endo- und exogene, aber auch durch physikalische Reize aktiviert und sorgt für eine Veränderung des Genexpressionsmusters. Die Hochregulation von Zytokinen und anderen Entzündungsmediatoren ist die Folge. Auf molekularer Ebene laufen diverse Reaktionen zeitgleich ab. Ein entscheidender Teil dieser 
Reaktion ist die Rekrutierung von Makrophagen. Makrophagen reagieren mit der Aktivierung von, unter anderem, Matrix-Metalloproteasen (MMP). MMP's sind Peptidasen, also Enzyme, welche die Spaltung von Peptidbindungen in Proteinen katalysieren. Bei Säugetieren sind 23 verschiedene MMP`s bekannt. Diese sind in der EZM lokalisiert und sorgen für deren Verfall. Außerdem provozieren die MMP's die Apoptose glatter Muskelzellen der Tunica media. Damit einhergehend kommt es zur Schwächung und Verdünnung der Gefäßwand und schließlich zur Formierung eines Aneurysmas bis hin zu dessen Ruptur (Chalouhi et al. 2013).

Die Signifikanz der MMP's wurde in einem Tierversuch mit Ratten belegt. Hierbei gaben die Forscher den Ratten „Tolylsam“, einen selektiven MMP-2- und MMP-9-Inhibitor. Dadurch wiesen sie eine Entstehung- und Wachstumsreduktion zerebraler Aneurysmen in den Tiermodellen nach (Aoki et al. 2007).

Makrophagen, zur Phagozytose befähigte Leukozyten, kommen in unterschiedlichsten Geweben vor. Ihre Aufgabe ist sowohl die Immunabwehr als auch die Förderung von Heilungsprozessen durch Abgabe diverser Wachstumsfaktoren. Je nach Lokalisation oder auch Zielstruktur werden sie unterschiedlich bezeichnet und sind phylogenetisch wahrscheinlich eines der ältesten Zellen des angeborenen Immunsystems. Sie werden von verschiedenen Lockstoffen, wie zum Beispiel Interferon-Y, aktiviert (Chemotaxis). Wenn Makrophagen die arterielle Gefäßwand infiltrieren, bewirken sie nicht ausschließlich die Hochregulation von MMPs und anderen Proteinasen, sondern rekrutieren zusätzlich pro-inflammatorische Zellen durch die Sezernierung von Wirksoffen wie TNFalpha oder Interleukinen. Es gibt verschiedene Makrophagen. Lange Zeit wurden sie in pro-inflammatorische M1- und anti-inflammatorische M2-Makrophagen eingeteilt. Eine Studie von Hasan et al. konnte in Biopsaten von rupturierten und unrupturierten Aneurysmen zeigen, dass das Verhältnis von M1- zu M2-Makrophagen signifikant unterschiedlich zwischen den beiden Biopsatgruppen war. Die Biopsate der rupturierten Aneurysmen zeigten ein Missverhältnis von M1 zu M2 und zwar zugunsten der proinflammatorischen M1-Zellen. Auch die Hochregulation von Mastzellen war bei den rupturierten Aneurysmen signifikant stärker ausgeprägt (Hasan et al. 2012). Diese Ergebnisse sind mittlerweile allerdings vorsichtig zu betrachten, da die seit Jahrzehnten vorherrschende Theorie der M1- und M2-Makrophagen grundsätzlich in Frage gestellt ist. Wissenschaftler haben die Existenz einer großen Anzahl verschiedener Makrophagen und damit eine größere Variabilität nachgewiesen. Das Modell von sich polarisierend gegenüberstehenden Makrophagen scheint damit überholt (Xue et al. 2014). 
Die glatten Muskelzellen der Tunica media tragen ebenfalls entscheidend zu der Formierung eines zerebralen Aneurysmas bei. Kommt es nun zu einer Schädigung des Endothels, immigrieren die glatten Muskelzellen in die Tunica intima und provozieren durch Proliferation eine Hyperplasie des Endothels. Dieses geschieht zu Ungunsten der Tunica media, also der Gefäßwandschicht, welche für die Integrität der Gefäßwand verantwortlich ist. Im Rahmen dieses Prozesses erleben die glatten Muskelzellen eine sog. Phänotyp-Modulation. Dabei werden aus differenzierten, kontraktilen Zellen entzündungsfördernde und die EZM abbauende Zellen. Dies geschieht über eine Hochregulation von Entzündungsmediatoren wie der MMP's, aber auch monocyte chemoattractant protein-1 (MCP-1) und Interleukinen. Ebenfalls kommt es zu einer Runterregulation der Protein- und mRNA-Synthese von zum Beispiel Myokardin (Nakajima et al. 2000; Starke et al. 2014b). Die Grundorganisation von glatten Muskelzellen in engen kompakten Banden ist aufgehoben. Auseinanderstehende, spinnenähnlich konfigurierte glatte Muskelzellen, welche nicht mehr in der Lage sind, Kollagene für die EZM zu synthetisieren, sind die Folge. Verstärkter Untergang von funktionell und morphologisch intakten glatten Muskelzellen der Tunica Media, bei verstärkter Apoptose durch höhere Caspase-Aktivität in der Gefäßwand, ist ein ebenfalls zu bedenkender Pathomechanismus des Rupturvorgangs eines Aneurysmas (Sakaki et al. 1997). TNF-alpha gilt als einer der Hauptinduktoren der phänotypischen Modulation und damit der Entstehung von zerebralen Aneurysmen. Er wirkt proinflammatorisch und proapoptotisch. Darüber hinaus steigert er die Produktion von reaktiven Sauerstoffspezien (ROS), rekrutiert Makrophagen in die Aneurysmawand und hemmt den kontraktilen Phänotyp der glatten Muskelzellen (Jayaraman et al. 2005; Starke et al. 2014a). Es konnte gezeigt werden, dass durch die Gabe des TNF-alpha-Inhibitors Infliximab die phänotypische Modulation nach Aneurysma Induktion invertiert werden konnte (Ali et al. 2013). Wie oben bereits erwähnt, fand sich in Biopsaten rupturierter Aneurysmen eine hohe Anzahl von Mastzellen. Dieses lässt darauf schließen, dass auch diese, hauptsächlich durch die Freisetzung von inflammatorischen Zytokinen, an der Pathogenese zerebraler Aneurysmarupturen beteiligt sind. Versuche an Mäusen mit Mastzell-Stabilisatoren konnten die Progression zerebraler Aneurysmen stoppen (Ishibashi et al. 2010).

Diversen Zytokinen konnte die Beteiligung an der Entstehung zerebraler Aneurysmen nachgewiesen werden. Sie sind entweder an der initialen endothelialen Dysfunktion (IL13, ROS, Angiotensin2, Phosphodiesterase, Prostaglandin E2 etc.), der phänotypischen 
Modulation glatter Muskelzellen (TNF-alpha, IL-1B, MMP's etc.), der Makrophagen Infiltration (TNF-alpha, IL-1ß, MCP-1, Interferon-Y, etc.) oder der Gefäßumstrukturierung und am Zelltods (MMP's, TNF-alpha, IL-1B, IL-6, ROS etc.) mitbeteiligt (Chalouhi et al. 2013).

Lysosomen, also ubiquitär in tierischen Zellen vorkommende Zellorganellen, haben die Funktion, Bipolymere in Monomere zu spalten. Sie bestehen aus in einer Biomembran verpackten, Verdauungsenzymen und stehen im Dienste der Heterophagie und Autophagie. Kleinloog et. al konnten eine signifikant höhere Expression lysosomaler Genloci in RNA rupturierter Aneurysmen nachweisen. Die Vermutung liegt nahe, das ein sog. lysosomaler Pathway zumindest zum Rupturvorgang, wenn nicht auch zur Formation von zerebralen Aneurysmen beiträgt (Kleinloog et al. 2016).

\subsubsection{Hämodynamischer Stress als Ursache der Aneurysmaformation}

Hämdodynamischer Stress, also eine gesteigerte Belastung des Gefäßsystems, ist der Hauptauslöser und Startpunkt des bereits beschriebenen Pathomechanismus zerebraler Aneurysmen. Dieser kann durch verschiedene Faktoren ausgelöst werden. Es gibt modifizierbare Risikofaktoren, die hämodynamischen Stress induzieren können. Dieses gilt hauptsächlich für die bekannten kardiovaskulären Risikofaktoren wie Hypertension, Hyperlipoproteinämie, Diabetes mellitus und das Rauchen von Tabak (Vlak et al. 2013). Die nicht-modifizierbaren Risikofaktoren sind genetische Prädispositionen, das weibliche Geschlecht sowie Infektionen und Traumen. Neben den hohen Inzidenzraten bei Patienten mit den erwähnten Risikofaktoren sprechen auch die Lokalisationen zerebraler Aneurysmen für das Vorhandensein hämodynamischen Stresses. So befinden sich Aneurysmen signifikant häufiger an Bifurkationen und Gefäßabgängen. Dort existiert eine erhöhte mechanische Belastung auf die Gefäßwand, vor allem auf die Endothelzellen. Der Schädigung des Endothels folgt die beschriebene lokale Entzündungsreaktion (Jamous et al. 2007). Unklar ist, wie sich die Stärke der mechanischen Belastung, der sog. Scherstress, auf die Formierung zerebraler Aneurysmen auswirkt. Die einfache Formel, je stärker der Scherstress, desto höher die Gefahr der Formation und des Wachstums eines Aneurysmas, konnten Wissenschaftler widerlegen. Meng et al. publizierten, dass sowohl schwacher als auch sehr starker Scherstress zur Ausbildung von Aneurysmen beitragen kann. Allerdings geht dieses nur durch unterschiedliche Pathomechanismen. So soll schwacher Scherstress durch entzündliche Prozesse für große Aneurysmen des arterio- 
sklerotischen Typs verantwortlich sein. Starker Scherstress hingegen soll durch den primären Untergang der Zellwand, also Zellapoptose und MMP Produktion und damit einhergehende Ausdünnung der Tunica media, die Ausbildung kleiner Aneurysmen provozieren (Meng et al. 2014). Natürlich können in der Natur nicht beide Pathomechanismen als völlig unabhängig voneinander betrachtet werden. Viele zerebrale Aneurysmen sind daher ein Zusammenspiel beider.

\subsubsection{Genetische Aspekte der Aneurysmabildung}

In vielen Lehrbüchern werden genetische Prädispositionen als Ätiologie von zerebralen Aneurysmen aufgeführt. Belegt sind Zusammenhänge zwischen genetisch bedingten Erkrankungen und dem Auftreten zerebraler Aneurysmen, wie beispielsweise bei der autosomal dominanten polizystischen Nierenerkrankung. Aus Autopsiestudien an über 60jährigen Patienten weiß man, dass fast jeder vierte Erkrankte ein zerebrales Aneurysma entwickelte (Schievink et al. 1992). Weitere kongenitale Erkrankungen, die mit einem erhöhten Auftreten aneurysmatisch bedingten SAB's einhergehen, sind das Ehlers-Danlos-Syndrom (EDS) Typ 4 und die fibromuskuläre Dysplasie (FMD). Das EDS Typ 4 (auch als vaskulärer Typ bezeichnet) ist eine autosomal rezessiv vererbte Erkrankung und beruht auf einer Mutation des Kollagen-Typs 3. Bei der fibromuskulären Dysplasie kommt es zu einer nicht-entzündlichen Proliferation von Bindegewebe und glatten Muskelzellen in der arteriellen Gefäßwand, bislang unklarer Genese. Auch das Marfan-Syndrom, eine Mutation des Fibrillin-1-Gens, ist mit erhöhtem Auftreten von Aneurysmen assoziiert. Eine höhere Prävalenz von aSAB konnte bislang nicht bewiesen werden (van den Berg et al. 1996). Das Vorhandensein familiärer Häufungen bei der SAB spricht für die Existenz genetischer Prädispositionen. Verwandte ersten Grades, von Patienten mit vorrausgegangener $\mathrm{SAB}$, haben ein bis zu siebenfach erhöhtes Risiko ebenfalls eine $\mathrm{SAB}$ zu erleiden (Bromberg et al. 1995c). Im Durchschnitt erkrankt die spätere Generation 6,8 Jahre früher als das Vergleichskollektiv mit negativer Familienanamnese. Antizipation ist daher denkbar. Diese Erkenntnis und die Tatsache, dass überproportional häufig die $\mathrm{ACM}$ von Aneurysmen betroffen ist, veranlassten die Forschung, die familiäre $\mathrm{SAB}$ als Erkrankung mit eigener Entität zu betrachten. Erschwerend kommt hinzu, dass Patienten mit positiver Familienanamnese mit höherer Wahrscheinlichkeit ein schlechteres Outcome erleiden als Patienten mit sporadischer SAB (Bromberg et al. 1995a; Bromberg et al. 1995b). Dem gegenüber stehen Daten aus einer großen Zwillingstudie. In der Nordic Twin Study wurde nach genetisch epidemiologischen Daten der spontanen SAB gesucht. 
Die 79644 Zwillingspaare aus Dänemark, Schweden und Finnland umfassende Kohortenstudie konnte keine genetische Häufung aufzeigen. Es wurde so interpretiert, dass die SAB keinen genetischen Ursprung habe (Korja et al. 2010). Familiäre Häufungen erklären sich auch durch Sozialisierung im Umgang mit Gesundheit, also der vorgelebten Weitergabe von Lebensstilen und gesundheitspräventivem oder -schädlichem Verhalten. Auch wenn bislang kein eindeutiges Gen für die Entwicklung zerebraler Aneurysmen gefunden wurde, konnten in mehreren Studien prädisponierende Genloci für die aSAB entdeckt werden. Meist kodieren diese Genloci für Strukturproteine wie Kollagen, Elastin oder andere extrazellulären Matrix-Proteine (Ruigrok et al. 2005; Kurki et al. 2014).

\subsubsection{Das Rauchen als Risikofaktor für die Entstehung eines Aneurysmas.}

Als einer der stärksten vermeidbaren Risikofaktoren muss wie bereits belegt, auch bei der SAB, das Rauchen von Tabak erwähnt werden. Das Rauchen fördert nicht nur die Formation eines Aneurysmas, sondern erhöht auch das Rupturrisiko signifikant. Meist gilt das als Folge der gefäßschädigenden pro-inflammatorischen Inhaltsstoffe des Rauchens. Wer raucht, wirkt in allen bislang verstandenen Entstehungsschritten verstärkend auf deren Verlauf ein. Die Inhaltsstoffe des Tabaks induzieren hämodynamischen Stress und fördern dadurch die endotheliale Dysfunktion und Apoptose. Chalouhi et al. konnten zeigen, dass das Rauchen die phänotypische Modulation glatter Muskelzellen provoziert, Zytokine freisetzt und nicht zuletzt die gefäßwandschädigenden Sauerstoffradikale generiert (Chalouhi et al. 2012). Ein weiterer Risikofaktor, der durch das Rauchen begünstigt wird, ist die Entstehung eines arteriellen Hypertonus. Es kommt zu einer GröBenzunahme des Aneurysmas und dadurch zu einer fortschreitenden Gefäßwandausdünnung

\subsubsection{Das Rupturrisiko bei vorhandenem zerebralem Aneurysma}

Einer der wichtigsten Entscheidungsparameter für das therapeutische Vorgehen bei Patienten mit unrupturierten zerebralen Aneurysmen ist die Größe, welche in der Regel in mm Durchmesser angegeben wird. Hiervon hängt nicht nur die Entscheidung ab, ob therapiert wird, sondern unter anderem auch mit welchem Verfahren (interventionell oder chirurgisch) therapiert wird. Die Größe ist ein klares Entscheidungskriterium in den Leitlinien und geht proportional mit dem Rupturisiko einher (Wiebers et al. 2003; UCAS Japan Investigators et al. 2012). Die eben zitierte japanische Kohortenstudie belegte dies erneut. Die analysierten Aneurysmen wurden hierfür nach ihrer Größe eingeteilt und 
anhand der Hazard Ratio (HR) beurteilt. Die Hazard Ratio ist zu verstehen als Wahrscheinlichkeitsangabe einer Kohorte, bezogen auf eine Vergleichskohorte, dass ein Ereignis, in diesem Fall die Ruptur eines vorhandenen Aneurysmas, stattfinden kann. Ist die HR gleich eins, so ist die Wahrscheinlichkeit beider Vergleichskohorten, dass es zu einem Ereignis kommt, identisch. Bei einer HR größer eins ist die Wahrscheinlichkeit für die betrachtete Kohorte größer und bei kleineren Werten dementsprechend geringer. Als Referenzkohorte wurde das Rupturrisiko von unrupturierten Aneurysmen mit 3-4 mm Durchmesser genommen. Aneurysmen mit einem Durchmesser von 5-6 mm wiesen bereits eine $\mathrm{HR}$ von 1,13 auf, wohingegen Aneurysmen mit 7-9 mm schon eine HR von 3,35 haben. Die nächste Gruppe beinhaltete Aneurysmen von 10-24 mm Durchmesser und zeigte eine HR von 9,09. Für Riesenaneurysmen, also Aneurysmen größer als 25mm Durchmesser, errechnete sich eine HR von 76,26 bezogen auf die Referenzkohorte. Diese Kohortenanalyse zeigte nicht nur den Zusammenhang zwischen Größe und Rupturisiko auf, sondern konnte weitere Risiken bei vorhandenem Aneurysma ermitteln. Bei einem jährlichen Rupturrisiko von 0,95\% der japanischen Kohorte konnte sie aufzeigen, dass die Lokalisation und die Geometrie des Aneurysmas entscheidende Kriterien für dessen Rupturrisiko sind. So haben Aneurysmen der anterioren und posterioren Communicans-Arterien sowie Aneurysmen der A. basilaris ein signifikant erhöhtes Risiko zu rupturieren und damit eine SAB hervorzurufen als zum Beispiel Aneurysmen der ACM. Aneurysmen, die geometrisch einen Tochtersack aufweisen, haben zudem höhere Rupturraten (UCAS Japan Investigators et al. 2012). Aufgrund der Signifikanz der Konfiguration und Geometrie der Aneurysmen sind für den klinischen Alltag mehrere leicht $\mathrm{zu}$ berechnende Indices entworfen worden. Ein bedeutender Index ist der sog. Aspect Score. Er berechnet sich als Quotient aus der Höhe und der Halsbreite des Aneurysmas. Ujiie et.al prüften den Aspect Score auf seine prognostische Bedeutung bezogen auf das Rupturrisiko. Die Forschungsgruppe teilte die 129 Patienten mit rupturiertem Aneurysma und 72 Patienten mit unrupturiertem Aneurysma in 4 Gruppen ein. Die Aufteilung erfolgte nach Lokalisation der zerebralen Aneurysmen. 80\% der Patienten mit rupturierten Aneurysmen wiesen einen Aspekt Score von >1,6 auf, wohingegen das Patientenkollektive mit unrupturierten Aneurysmen zu 90\% einen Aspect Score <1,6 hatten. Diese Erkenntnis bringt dem Aspekt Score eine gewisse Bedeutung zu, da man ihn zur Abschätzung des Rupturrisikos zerebraler Aneurysmen nutzen kann (Ujiie et al. 2001). Über den geeignetsten Schwellenwert des Aspect Scores gibt es derzeit unterschiedliche Meinungen in der Wissenschaft. Ryu et. al analysierten aus einer Datenbank 204 verschiedene 
Aneurysmen bei 195 Patienten auf geometrisch anatomische Eigenschaften. Sie haben dafür 109 unrupturierte Aneurysmen und 105 rupturierte Aneurysmen ausgewertet. Ryu et. al maßen den Aneurysmahals, die Höhe, Breite und Tiefe des Aneurysmas und teilten sie nach ihrer Lokalisation, ihrer Größe und ihrer geometrischen Form ein. Nach der Akquise erstellte die Forschungsgruppe verschiedene Indices. Damit bewiesen sie, dass nicht nur die einzeln bestimmten Werte, wie zum Beispiel die Größe oder das Volumen, sondern auch das Verhältnis der Messwerte zueinander relevant für das Rupturrisiko sein können. Die untersuchten Indices, die einen signifikanten Messunterschied zwischen rupturierten und unrupturierten Aneurysmen aufzeigten und damit als relevante Scores angesehen werden können, waren der oben erwähnte Aspect Score, der Quotient aus Volumen des Aneurysmas und der Fläche des Aneurysmahalses, der Quotient aus Höhe und Breite des Aneurysmas und das Verhältnis aus der Breite des Aneurysmas und der Breite des Aneurysmahalses, der sog. Bottleneck-Faktor (Ryu et al. 2010). Ein erhöhter Bottleneck-Faktor konnte ebenfalls von anderen Wissenschaftlern, zusammen mit dem Höhe-zu-Breite Verhältnisses, als signifikant belegt werden. In der selben Analyse von Hoh et al. wurden allerdings andere Indices, zum Beispiel der Aspekt Score, aber auch weitere Indices, welche hier nicht erwähnt sind, untersucht. Zumindest in dieser Publikation konnten sie nicht als geeignete Parameter bewiesen werden (Hoh et al. 2007). Ein anderer stark untersuchter Index ist die sog. Size Ratio, also der Quotient aus Aneurysma und der Gefäßdurchmesser der betroffenen Gefäße. Wie aus der Definition ableitbar, können für diesen Score mehrere Gefäße betreffen. Kashiwazaki et. al verglichen die Größe und die Size Ratio von kleinen Aneurysmen ( $<5 \mathrm{~mm})$. Sie kamen zu dem Ergebnis, dass die Size Ratio besser, als die eigentliche Größe kleiner Aneurysmen, das Rupturrisiko aufzeigen kann (Kashiwazaki et al. 2013). Die Size Ratio ist damit gut für die Therapieplanung kleiner Aneurysmen geeignet. Über die geometrischen Indices und deren Aussagekraft ist man sich unter Wissenschaftlern nicht einig. Es belegen dennoch viele Untersuchungen, dass die gängigen Indices, wie der Aspekt Score, Size Ratio und der Bottleneck-Faktor, häufig mit Rupturrisiken korrelieren. Sie sind einfach zu bestimmen und eignen sie sich gut als Zusatzinformation für die Therapieplanung bei Patienten mit unrupturierten zerebralen Aneurysmen.

Es besteht ein starkes Risiko bei Patienten, die ein Wachstum bei vorhandenem unrupturiertem Aneurysma aufweisen. Alle Prozesse, die das Wachstum eines Aneurysmas fördern, wie zum Beispiel das Rauchen, sind dementsprechend als Risikofaktoren miteinzu- 
beziehen. In einer großen Studie konnte diese Annahme mit Zahlen belegt werden. Villablanca et. al konnten aufzeigen, dass wachsende Aneurysmen in 2,4 \% rissen, wohingegen stagnierte, nicht wachsende Aneurysmen nur in 0,2 \% der Fälle rupturierten. Mit anderen Worten haben wachsende Aneurysmen damit ein 12-fach höheres Rupturrisiko als nicht-wachsende. Rauchen und die Ausgangsgröße des Aneurysmas sind demnach Kofaktoren, welche eine Ruptur begünstigen (Villablanca et al. 2013). So ist dringend zur Therapie zu raten, wenn Patienten ein Wachstum in einer Kontrollbildgebung aufweisen. In einer weiteren Studie konnten der Quotient aus Aneurysmadom zum Aneurysmahals als weiterer Prädiktor eines Größenzuwachses und damit einer Ruptur des Aneurysmas zusätzlich zu dem Rauchen und der Initialgröße des Aneurysmas belegt werden (Bor et al. 2015).

Die WHO MONIKA stroke study untersuchte das Auftreten der nicht-traumatischen $\mathrm{SAB}$ in 11 verschiedenen Populationen aus Europa und China. Die Inzidenz variierte von 2,0 auf 100000 Einwohner in China, bis auf 22,5 auf 100000 Einwohner in Finnland. Dementsprechend lässt sich ableiten, dass die Herkunft oder Region mit unterschiedlichen Rupturrisiken für Aneurysmen einhergeht. Ebenfalls wurde belegt, dass Frauen im Vergleich zu Männern - ein ca. 1,6-fach erhöhtes Risiko haben, eine SAB zu erleiden (Ingall et al. 2000).

2013 publizierten Wissenschaftler ein Punktesystem, welches darauf abzielt, das Wachstum von Aneurysmen und damit einhergehend auch das Rupturrisiko unter Berücksichtigung mehrerer Prädiktoren anzugeben, den PHASES Score. Die Buchstaben beinhalten den betrachteten Prädiktor. Das „P“s steht für population (engl.). Demnach bekommen Amerikaner und Europäer (außer Finnen) 0 Punkte. Japaner hingegen erhalten 3 und Finnen 5 Punkte. Studien belegten höhere Prävalenzen für nicht traumatische SAB's in diesen Regionen. Das „H“ steht für den Risikofaktor Hypertension und ergibt bei Vorhandensein einen Punkt. "A“" steht für Alter (age). Bei Patienten über 70 Jahren gibt es einen Punkt. Das erste „S“ steht für die Größe des Aneurysmas (size). Aneurysmen kleiner als 7 mm Durchmesser bekommen 0 Punkte, Aneurysmen zwischen 7-9,9 mm Durchmesser erhalten 3 Punkte, Aneurysmen zwischen 10,0 und 19,9 bekommen 6 und ab einer Größe von 20 mm Durchmesser bekommt man 10 Punkte zugeschrieben. Das „E“ steht für eine stattgehabte $\mathrm{SAB}$ aus einem anderen Aneurysma (engl. earlier $S A B$ from another aneurysm) und gibt bei stattgehabter SAB einen Punkt. Das andere „S“ steht für die Lokalisation des Aneurysmas, genauer, welches Trägergefäß betroffen ist (site of aneurysm). Für die ACI gibt es 0 Punkte. Für die ACM gibt es 2 und bei einem Aneurysma des 
vorderen- (beinhalten die anterioren Arterien, die Arteria communicans anterior, und der Arteria pericallosa.) und hinteren Stromgebiet (beinhalten die posterioren Arterien sowie die Arteriae vertebralis, -basilaris und -cerrebellaris) erhält man 4 Punkte. Aus der Summe der Punkte errechnet sich dann das 5-Jahres-Rupturrisiko (Greving et al. 2014).

\subsubsection{Pathophysiologie der aneurysmatischen SAB}

Kommt es zur Ruptur eines zerebralen Aneurysmas, so strömt Blut mit arteriellem Druck in den Subarachnoidalraum. Das Blut bewirkt eine Tamponierung der äußeren Liquorräume. Es verstopft die Foramina Luschkae und das Foramen Magendii, die Liquordurchtrittstellen des vierten Ventrikels. Die Liquorzirkulation ist dadurch gestört. Das Blut behindert die Granulationes arachnoideales dabei, den Liquor zur resorbieren. Die Granulationes arachnoidales sind kleine Ausstülpungen der Arachnoidea mater, die durch die Dura mater ziehen. Diese Protrusionen fungieren als Klappen, die nur eine Flussrichtung zulassen, nämlich die Resorption des Liquors in das venöse Gefäßsystem. Auf die Behinderung des Liquorflusses durch das hereinströmende Blut folgt ein massiver Anstieg des intrakraniellen Drucks (ICP). Der hohe ICP behindert den venösen Blutabfluss. Dadurch kommt es zu einem Hirnödem, welches sein Maximum nach ca. einer Woche findet. In schweren Fällen kommt es schon innerhalb der ersten 24 Stunden zu einem akuten Hydrozephalus (Masuhr et al. 2013).

Der zerebrale Perfusionsdruck (CPP) ist abhängig von dem ICP und dem mittleren arteriellen Druck (MAD). Mathematisch ausgedrückt besteht folgender Zusammenhang: $\mathrm{CPP}=\mathrm{MAD}-\mathrm{ICP}$

Steigt nun der ICP bei einer SAB an, so sinkt der CPP. Es besteht die Gefahr der zerebralen Ischämie. Der Körper kompensiert dies mit der Erhöhung des systolischen Blutdrucks auf Spitzenwerte von bis zu $300 \mathrm{mmHg}$. Dieser Mechanismus wurde erstmals von dem amerikanischen Neurologen und Neurochirurgen Harvey Cushing (1869-1939) beschrieben und wird seither als Cushing-Reflex bezeichnet. Das Herz kompensiert die immense Kraftanstrengung häufig mit einer Bradykardie. Fast alle EKG-Veränderungen sind im Rahmen dieser reaktiven arteriellen Hypertonie denkbar, was in manchen Fällen den Ausschluss eines akuten Koronarsyndroms erschwert (Böcher-Schwarz et al. 2014). 
Die venöse Abflussstörung zusammen mit dem hohen arteriellen Druck erhöhen das Risiko einer sekundären hämorrhagischen Infarzierung. Eine hämorrhagische Infarzierung ist eine Gewebenekrose aufgrund der venösen Abflussstörung. Es muss klar von einem tatsächlichen Infarktgeschehen abgegrenzt werden.

Eine SAB führt in 70\% der Fälle zu einer Gefäßkonstriktion. Der Vasospasmus kann lokal oder global auftreten und wird wahrscheinlich durch die Freisetzung vasoaktiver Substanzen, wie Serotonin oder Prostaglandine, verursacht. Der genaue Pathomechanismus, der für die Entstehung des Vasospasmus verantwortlich ist, ist derzeit noch Bestandteil wissenschaftlicher Forschung. Wie auch bei anderen Verletzungen der Gefäßwand setzen nach dem Riss des Aneurysmas Heilungsprozesse ein. Die Spontanthrombosierung und lokale Gefäßkonstriktion des Aneurysmas unterstützen die Blutstillung. Kommt es nun zur Thrombusbildung am Aneurysma, so sorgt die physiologische Fibrinolyse für die hohen Prozentzahlen an Rezidivblutungen. Diese Rezidivblutungen treten in 20-30\% der Fälle innerhalb des ersten Monats nach ereigneter SAB auf. Als Folge der ersten SAB haben sich die Leptomeningen (weichen Hirnhäute) verklebt. Das nun als zweite SAB einströmende Blut kann sich durch die verklebenden Hirnhäute nicht mehr ausbreiten und es kommt eher zur Blutung in die Hirnsubstanz (Masuhr et al. 2013). Die folgende Tabelle nach Masuhr et al. stellt den Pathomechanismus und die daraus resultierenden Komplikationen in Zusammenhang und gibt Häufigkeitsangaben der Komplikationen innerhalb der ersten Tage nach einer SAB an.

\begin{tabular}{|l|l|l|}
\hline $\begin{array}{l}\text { Pathophysiologie wäh- } \\
\text { rend SAB }\end{array}$ & $\begin{array}{l}\text { Folge und Komplika- } \\
\text { tion }\end{array}$ & $\begin{array}{l}\text { Häufigkeit der Kom- } \\
\text { plikation innerhalb } \\
\text { der ersten Tage nach } \\
\text { SAB }\end{array}$ \\
\hline Erhöhter ICP & Hirnödem & $50 \%$ \\
\hline $\begin{array}{l}\text { Liquorzirkulations- und } \\
\text { resorptionsstörung }\end{array}$ & Akuter Hydrozephalus & $15-20 \%$ \\
\hline \begin{tabular}{l} 
Vasospasmus \\
\hline
\end{tabular} & Hirninfarkt & $30 \%$ \\
\hline
\end{tabular}


Tabelle 1: Pathomechanismus und die resultierenden Komplikationen der SAB; modifiziert nach Masuhr et al. 2013 


\subsection{Akutsymptome und klinische Zeichen der SAB}

Wegweisendes Leitsymptom ist der sog. Vernichtungskopfschmer als ein binnen Sekunden eintretender Kopfschmerz von bislang unbekannter Massivität. Häufig ist dieser Vernichtungskopfschmerz gepaart mit fokal neurologischen Ausfällen, Nackenschmerzen oder -steifheit, Übelkeit und Erbrechen. Klinische Hinweise bei Verdacht auf SAB können Menigismus, Vigilanzminderung bis hin zum Bewusstseinsverlust, Hirnnervenausfälle wie zum Beispiel eine Okkulomotoriusparese, aber auch Glaskörpereinblutungen sein (DGN 2017a).

Wie häufig in der Medizin können Symptome teilweise schwach oder nicht vorhanden sein. Dieses führt dazu, dass die SAB in $5-10 \%$ der Fälle klinisch übersehen wird. Im Allgemeinen muss man immer an eine SAB denken, sobald der Patient plötzlichen Kopfschmerz zusammen mit Hirndrucksymptomen oder neurologischen Defiziten präsentiert.

\subsection{Präklinisches und klinisches Vorgehen bei Akutsymptomen der SAB}

Die SAB ist eine stark lebensbedrohliche Erkrankung. Präsentiert sich ein Mensch mit den oben genannten Symptomen, so muss sofort medizinische Hilfe, im präklinischen sofort der Notarzt, alarmiert werden. Präklinisch sollten diese Patienten nach kurzer neurologischer Einschätzung umgehend, bei andauernder Überwachung der Vitalzeichen durch einen Arzt (zumindest in der Bundesrepublik Deutschland), in die nächste geeignete Klinik gebracht werden. Der Patient sollte der Stroke Unit der Zielklinik als Verdacht auf Apoplex vorangemeldet werden. Neben den kardialen Risikofaktoren ist das Erfragen von Kopfschmerzen innerhalb der letzten Tage und Wochen sinnvoll. 25\% der Patienten haben eine sog. Warnblutung, eine kleine Einblutung in den Subarachnoidalraum, welche sich Wochen bis Tage als Prodromie einer SAB in Form von Kopfschmerzen, darstellt. In vielen Fällen werden diese sowohl von Patienten als auch von Ärzten als harmlos fehlinterpretiert. Die zielgerichtete ausführliche neurologische Untersuchung sollte spätestens beim Eintreffen in der Zielklinik erfolgen. Der diensthabende Neurologe/Neurochirurg muss dabei auf die Bewusstseinslage und fokal-neurologische Defizite wie Paresen oder Aphasien achten. Das Vorliegen von Meningismus muss sowohl beim wachen als auch beim bewusstlosen Patienten überprüft werden. 
Auch Pyramidenbahnzeichen wie der Babinski-Reflex sollten geprüft werden. Das Setzen von Schmerzreizen bei bewusstlosen Patienten dient der Tiefeneinschätzung der Bewusstlosigkeit. Neben der neurologischen Untersuchung dürfen Routineuntersuchungen wie die Bestimmung des Blutzuckers oder die dauerhafte Überwachung der Vitalzeichen, nicht fehlen. Die klinische Untersuchung und die daraus folgende Differentialdiagnose bestimmen maßgeblich das weitere klinische Vorgehen. Als Hilfestellung für das Einschätzen des Patientenzustands wurden Klassifikationen zur SAB ausgearbeitet. Sie sind leicht eruierbar und sollten von den Klinikern als objektives Instrument genutzt werden.

\subsection{Klassifikationen der SAB}

Es gibt zwei führende Klassifikationen der SAB. Zum einen ist das die Klassifikation, die nach ihren Erstellern Hunt und Hess benannt ist. Zum anderen gibt es die Klassifikation der World Federation of Neurosurgical Societies, kurz WFNS. Beide sind dafür gedacht, nicht nur die Schwere der SAB, sondern dadurch auch die daraus resultierende Prognose einschätzen zu können.

Hunt und Hess publizierten 1968 ihre Klassifikation. Dabei teilen sie die SAB in fünf Schweregrade ein, wobei sie sich ausschließlich an der Klinik des Patienten orientieren. Der Schweregrad der Hunt-und- Hess-Klassifikation korreliert mit der Prognose des Patienten. Bei schwerer Systemerkrankung verschlechtert sich der Grad um eine Position (Hunt und Hess 1968).

Tabelle 2: SAB nach 5 klinischen Schweregraden eingeordnet; modifiziert nach Hunt und Hess 1968

\begin{tabular}{|l|l|}
\hline Grad & Symptomatik \\
\hline 1 & $\begin{array}{l}\text { Asymptomatisch, evtl. leichter Kopfschmerz/Meningismus bei inzidentel- } \\
\text { lem Aneurysma }\end{array}$ \\
\hline 2 & $\begin{array}{l}\text { Moderate bis schwere Kopfschmerzen, Meningismus aber keine neurologi- } \\
\text { schen Defizite mit Ausnahme von Hinnervenparesen }\end{array}$ \\
\hline 3 & \begin{tabular}{l} 
Somnolenz oder Verwirrtheit und mildes neurologisches Defizit \\
\hline 4
\end{tabular} \\
\hline 5 & $\begin{array}{l}\text { Sopor, Hemiparese (moderat oder schwer), beginnende Dezerebration, ve- } \\
\text { Koma, manifeste Zeichen der Dezerebration, moribundes Erscheinungsbild }\end{array}$ \\
\hline
\end{tabular}


Die Klassifikation nach WFNS ist eine Gradeinteilung, die sich nach der Anzahl der Punkte der Glasgow Coma Skale (GCS) und dem Vorhandensein motorischer Defizite richtet. Die GCS ist 1974 eigentlich mit dem Ziel publiziert worden, den Schweregrad eines Schädel-Hirn-Traumas (SHT) zu klassifizieren. Mittlerweile wird sie in verschiedensten Fällen zur Einschätzung des Bewusstseins herangezogen. Dabei vergibt die GCS Punkte in drei verschiedenen Kategorien, die nachfolgend dargelegt werden. Dem Öffnen der Augen (1-4 Punkte), der besten verbalen Reaktion (1-5 Punkte) und der besten motorischen Reaktion (1-6 Punkte) (Teasdale und Jennett 1974).

Tabelle 3: Glascow Coma Scale; modifiziert nach Teasdale und Jennett 1974

\begin{tabular}{|c|c|c|}
\hline Augen öffnen & $\begin{array}{l}\text { - } \text { Spontan } \\
\text { - } \text { auf Ansprache } \\
\text { - } \text { auf Schmerzreiz } \\
\text { - } \quad \text { kein Augen Öffnen }\end{array}$ & $\begin{array}{l}4 \\
3 \\
2 \\
1\end{array}$ \\
\hline verbale Reaktion & $\begin{array}{l}\text { - } \text { orientiert } \\
\text { - } \text { desorientiert } \\
\text { - } \text { inadäquat } \\
\text { - unartikuliert } \\
\text { - keine }\end{array}$ & $\begin{array}{l}5 \\
4 \\
3 \\
2 \\
1\end{array}$ \\
\hline $\begin{array}{l}\text { motorische } \\
\text { Reaktion }\end{array}$ & $\begin{array}{ll}\text { - } & \text { folgt Aufforderungen } \\
\text { - } & \text { gezielte Schmerzabwehr } \\
\text { - } & \text { ungezielte Schmerzabwehr } \\
\text { - } & \text { Beugereaktion } \\
\text { - } & \text { Streckreaktion } \\
\text { - } & \text { Keine }\end{array}$ & 1 \\
\hline
\end{tabular}

Ein GCS-Wert von 3-8 bedeutet entweder ein schweres SHT oder eine schwere Bewusstlosigkeit bis zum Koma. Patienten mit solchen Werten sollten in den meisten Fällen intubiert werden, um den Atemweg zu sichern. Einen GCS-Wert von 9-12 haben Patienten mit einer mittelschweren Bewusstseinsstörung oder SHT. Bei einem GCS-Wert von 1215 besteht entweder keine oder eine leichte Bewusstseinsstörung oder lediglich ein leichtes SHT. 
Der WFNS Score bedient sich fast ausschließlich der GCS. Lediglich zwischen Grad Zwei und Drei unterscheidet der WFNS Score den Schweregrad einer SAB durch ein anderes Kriterium als des GCS-Werts (Teasdale et al. 1988).

Tabelle 4: WFNS-Score, modifiziert nach Teasdale et al. 1988

\begin{tabular}{|l|l|l|}
\hline WFNS-Grade & GCS-Wert & Motorisches Defizit \\
\hline 1 & 15 & nicht vorhanden \\
\hline 2 & $13-14$ & nicht vorhanden \\
\hline 3 & $13-14$ & vorhanden \\
\hline 4 & $7-12$ & nicht vorhanden oder vorhanden \\
\hline 5 & $3-6$ & nicht vorhanden oder vorhanden \\
\hline
\end{tabular}

Das Benutzen der Klassifikationen ist hilfreich und sinnvoll, ersetzt jedoch nicht die genaue Dokumentation der patientenspezifischen Symptome und klinischen Zeichen. Diese sollten zur Verlaufskontrolle regelmäßig erhoben und dokumentiert werden.

\subsection{Apparative und interventionelle Diagnostik}

Besteht nach der klinischen Untersuchung der Verdacht auf eine SAB, so stellt die kranielle Computertomographie (CCT) die Diagnostik der ersten Wahl dar und wird auch als solche von den DGN-Leitlinien (2013) empfohlen. Die CCT ist ubiquitär verfügbar und hat innerhalb der ersten 24 Stunden eine Sensitivität von mehr als 95\% (van der Wee et al. 1995). Die Sensitivität nimmt mit dem zeitlichen Abstand zur Blutung stetig ab. Frisches Blut im Subarachnoidalraum stellt sich meist in den basalen Zysternen oder auch in den kortikalen Sulci hyperdens dar. Durch die CCT können Rückschlüsse auf das Vorhandensein einer Liquorzirkulations- oder resorptionsstörungen getroffen werden. Es kann die klinisch oft schwierige Differenzierung zwischen ischämischem oder hämorrhagischem Geschehen meist sicher getroffen werden. Zusätzlich kann sie erste Hinweise auf die Lokalisation der Blutungsquelle geben. Die qualitative Einschätzung der Schwere einer SAB in der Computertomographie wird durch die Klassifikation nach Fisher realisiert (Fisher et al. 1980). 
Tabelle 5: Fisher-Klassifikation zur qualitativen Einschätzung der SAB in der Comptertomographie; modifiziert nach Fisher et al. 1980

\begin{tabular}{|l|l|}
\hline $\begin{array}{l}\text { Fischer- } \\
\text { Grad }\end{array}$ & Befund im CT \\
\hline 1 & Kein sichtbares Blut im CT \\
\hline 2 & $\begin{array}{l}\text { Das Blut ist entweder diffus sichtbar oder es sind Blutkoagel } \\
<1 \mathrm{~mm} \text { Breite in den Inselzisternen sichtbar, dem Interhemi- } \\
\text { sphärenspalt oder der Cisterna ambiens. }\end{array}$ \\
\hline 3 & $\begin{array}{l}\text { Blutkoagel }>1 \text { mm Breite in den Inselzisternen, dem Interhe- } \\
\text { misphärenspalt oder der Cisterna ambiens }\end{array}$ \\
\hline 4 & $\begin{array}{l}\text { Intrazerebrale oder intraventrikuläre Blutkoagel mit oder ohne } \\
\text { SAB }\end{array}$ \\
\hline
\end{tabular}

Die CT-Angiographie (CT-A) gewann in den letzten Jahren immer größere Bedeutung. Durch dieses Kontrastmittel-gestützte Verfahren ist es in der Regel möglich, die Aneurysmen ohne großen Zeitaufwand darzustellen. Selbst geometrische Relationen des Aneurysmas lassen sich durch die 3D-Rekonstruktion aufzeigen (Böcher-Schwarz et al. 2014) Trotz der hohen Sensitivität der CT-A von über 99\% ist sie derzeit kein Bestandteil der DGN-Leitlinien.

Demnach soll bei Verdacht auf eine SAB und einer negativen CCT nach 8-12 Stunden eine Lumbalpunktion folgen. Die Lumbalpunktion stellte bis in die 1970er Jahre den Goldstandard der SAB-Diagnostik dar. Die Lumbalpunktion kann sensitiver als die CCT sein, wenn zum Beispiel die Blutmenge zu gering ist, um in der Bildgebung hyperdens zu erscheinen. Außerdem stellt sich in der CCT sieben Tage altes Blut isodens und damit quasi unsichtbar dar. Zusätzlich kann auch die spinale SAB durch eine Lumbalpunktion erkannt werden. Eine Lumbalpunktion darf ausschließlich bei Patienten erfolgen, die keinerlei Zeichen eines erhöhten intrakraniellen Drucks aufweisen. Durch die Reduktion des Drucks im spinalen Subarachnoidalraum könnte der Druckgradient zum kraniellen Subarachnoidalraum sich so vergrößern, dass Hirnparenchym nach kaudal gesogen würde. Die Wartezeit von 8-12 Stunden nach Symptombeginn wird zur Vermeidung falsch positiver Ergebnisse empfohlen. Nach dieser Zeit lässt sich eine Xantochromie klar von iatrogenem Blut im Liquor abgrenzen. Die Xantochromie, also Gelbfärbung, 
entsteht durch den Abbau des Hämoglobins. Die DGN-Leitlinien sehen für die Untersuchung des Liquors die Zentrifugation des Liquors, mit anschließender Begutachtung des gelben Überstandes vor einem weißen Hintergrund durch einen erfahrenen Kliniker als ausreichend an und beziehen sich dabei auf eine Studie von Linn et al., in der 51 Kliniker und 51 Studenten Proben beurteilen sollten. Das Ergebnis der Studie sagt, dass wenn ein gelber Überstand sichtbar ist, die SAB wie auch andere Differentialdiagnosen, welche eine Xantochromie im Liquor hervorrufen können, wahrscheinlich sind. Kommt es hingegen nicht zur makroskopisch sichtbaren Xanthochromie, kann eine SAB ausgeschlossen werden (Linn et al. 2005). Weitere wissenschaftliche Arbeiten empfehlen die Verwendung eines Spektrophotometers zur Analyse des Liquors. Die photometrische Messung der Lichtabsorption des Bilirubins, aber auch des Oxyhämoglobins und Methämoglobins ist sensitiver und spezifischer als die visuelle Beurteilung (Vermeulen und van Gijn 1990). Das Bilirubin hält sich zudem mehrere Tage im Liquor. Die spezifische Zelldifferenzierung des Liquors stellt wohl die am längsten eine SAB nachweisbare Methode der Liquoruntersuchung dar. Selbst nach mehreren Wochen finden sich nämlich noch Siderophagen, also Makrophagen, welche Eiseneinschlüsse durch den Abbau der Erythrozyten aufweisen (Böcher-Schwarz et al. 2014).

Ist durch die CCT der Nachweis frischen Blutes im Subarachnoidalraum gelungen, so folgt eine kathetergestützte Panangiographie zum Nachweis möglicher Aneurysmen. Die Panangiographie oder auch Vier-Gefäß-Angiographie ist der Goldstandard der Diagnostik intrakranieller Aneurysmen. Aus dem Befund können sich therapeutische Konsequenzen erschließen. Es sollten möglichst alle Arterien dargestellt werden, da sich in 2030\% der Fälle multiple Aneurysmen detektieren lassen. Dafür sollen beide Arteriae Carotides und beide Arteriae vertebralis in seitlichen, anterior-posterioren und in zwei horizontalen Schrägachsen abgebildet werden (Böcher-Schwarz et al. 2014). Als technischer Goldstandard ist dafür heutzutage die digitale Subtraktionsangiographie (DSA) geeignet (Kirkpatrick 2002). Bei der DSA wird initial eine Röntgenleeraufnahme angefertigt. Nach Verabreichung des jodhaltigen Kontrastmittels stellen sich die Gefäße hyperdens dar. Die Leeraufnahme wird nun über eine Computersoftware von der Kontrastmittelaufnahme subtrahiert, sodass eine reine Darstellung des Gefäßsystems möglich ist. Die DSA hat den Vorteil, dass -neben der guten Bildqualität- sich bei einem positivem Aneurysmanachweis eine direkte endovaskuläre Therapie anschließen könnte.

Da auch spinale Gefäßmalfomationen eine SAB auslösen können, empfiehlt es sich, bei negativem Angiographiebefund eine spinale Kernspintomographie zu fahren. Insgesamt 
spielt die Magnetresonanztomographie (MRT) keine Rolle in der Akutdiagnostik der SAB. Sie eignet sich aber in Form einer MR-Angiographie (MRA) als Screeninguntersuchung bei kleinen inzidentellen Aneurysmen, da sie ohne Strahlenbelastung als nichtinvasives Verfahren mit ähnlicher Sensitivität betrachtet wird (Buhk et al. 2009).

\subsection{Therapieoptionen bei zerebralen Aneurysmen und aSAB}

Sir Victor Horsley (1857-1916), ein britischer Physiologe und Neurologe, war ein Pionier im Bereich der Hirnchirurgie. Er gilt als der erste, der ein intrakranielles Aneurysma therapiert hat. 1885 vermutete Horsley während eines neurochirurgischen Eingriffs bei Verdacht auf einen Hypophysentumor ein Aneurysma der Schädelbasis mit Kompression des Chiasma-Optikums. Horsley ligierte beide Karotisarterien am Hals. Der Patient starb Jahre später und die Obduktion bestätigte Horsleys Vermutung (Ljunggren et al. 1993). Nun über 100 Jahre später, wurden bereits mehrere Tausend Patienten mit zerebralen Aneurysmen behandelt. Lange war die Therapie zerebraler Aneurysmen alleinige Aufgabe der Neurochirurgie. António Caetano de Abreu Freire Egas Moniz (1874-1955) war ein portugiesischer Neurologe, Politiker und Medizin-Nobelpreisträger, der aufgrund seiner rabiaten und wenig evidenzbasierten Psychochirurgie heutzutage sehr umstritten ist. Ihm gelang im Jahr 1932, zusammen mit dem portugiesischen Arzt Almeida Lima, die erste angiographische Darstellung intrakranieller Aneurysmen. Durch die Optimierung der Angiographie und der Kathetertechnik, vor allem durch den schwedischen Radiologen Sven-Ivar Seldinger, keimte früh der Wunsch auf, intrakranielle Aneurysmen endovaskulär zu therapieren. 1990 wurde durch den italienischen Neurochirurgen Dr. Guido Guglielmi erstmals ein Aneurysma eines Patienten endovaskulär okkludiert. Mittlerweile wird ein großer Teil der Patienten in spezialisierten Zentren durch Neuroradiologen endovaskulär behandelt. Die gängigsten Verfahren zur Behandlung intrakranieller Aneurysmen sind heutzutage das Angiographie-gestützte Coiling und das offenchirurgische Clipping.

\subsubsection{Die endovaskulären Behandlungsmöglichkeiten intrakranieller Aneurysmen}

Schon seit den 1980er Jahren wurden Aneurysmen endovaskulär therapiert. Dies ist prinzipiell möglich und zwar durch den Verschluss des aneurysmatragenden Gefäßes (parent vessel occlusion, PVO) über die Rekonstruktion des aneurysmatragenden Gefäßes oder der endosakkulären Okklusion wie beim Coiling. Durch den medizinischen Fortschritt gibt es heutzutage eine Vielzahl von verschiedenen endovaskulärenen Medizinprodukten, 
sog. Devices wie Coils (Platinsspiralen), Stents, WEB-Devices oder Flow-Diverter. Entscheidendes Indikationskriterium für die endovaskuläre Therapie sind Größe und Morphologie des Aneurysmas. Multimorbiden Patienten mit einem insgesamt reduzierten Allgemeinzustand wird häufiger zur endovaskulären Therapie geraten, da der Eingriff als minimal-invasiver gilt. Die Therapie sollte zeitnah nach dem Ereignis erfolgen. Die Gabe von ASS und Clopidogrel vor der endovaskulären Behandlung bietet sich an, um die Möglichkeit der Stent-Implantation zu behalten. Dieses gilt aber nur, sofern keine anderen Kontraindikationen dies verbieten. Neuste Verfahren wie zum Beispiel das WEBDevice benötigen keine postinterventionelle Thrombozytenaggregation, was ein Vorteil bei der SAB darstellt.

Beim Coiling, auch endovaskuläre Aneurysma-Okklusion genannt, wird durch einen Mikrokatheter eine Platinspirale bis in den Aneurysmasack vorgeschoben und endovaskulär platziert. Der Mikrokatheter wird angiographisch beobachtet, während er, meist transfemoral, durch das Gefäßsystem bis in die Gehirnarterien geführt wird. Die Platinspiralen lösen sich je nach Fabrikat elektrolytisch, elektrothermisch oder mechanisch von dem Mikrokatheter ab. Durch den sog. Formgedächtniseffekt nehmen die Platinspiralen nach Ablösung wieder ihre ursprüngliche Konfiguration ein und füllen so das Aneurysma aus. Der Formgedächtniseffekt beschreibt die Eigenschaft von Formgedächtnislegierungen (einer Gruppe von Metallen) in zwei Konfigurationen existieren zu können. Die Platinspiralen werden in einer bestimmten Konfiguration hergestellt. Nachdem der Neuroradiologe sie während des angiographischen Eingriffs in einer gestreckten, durch den Führungskatheter begrenzten Form eingeführt hat, nehmen die Platinspiralen beim Ablösevorgang ihre ursprüngliche Struktur an. Das vermeintliche „Erinnern“ an die ursprüngliche Konfiguration ist namensgebend für den Formgedächtniseffekt (Shape-Memory-Effekt). Der erste „Coil“ (Platinspirale) sollte so ausgewählt werden, dass seine vorgeprägte 3D-Konfiguration dem Durchmesser des Aneurysmas entspricht. Die Coils, ebenso wie andere Devices, sind in der Regel in Millimeter-Größenabständen erhältlich. Es werden je nach Aneurysmagröße mehrere Platinspiralen verwendet, die sich endovaskulär zu einem Knäul verflechten. Der Effekt liegt in der hervorgerufenen Stase innerhalb des Aneurysmasacks. Die Stase verursacht eine Thrombosierung des Aneurysmas. Da die Blutzirkulation im Aneurysma unterbunden ist, folgt innerhalb einiger Wochen eine Fibrosierung des Aneurysmas mit Endothelialisierung der ehemaligen Aneurysmagrenze. Dr. Guido Guglielmi publizierte 1991 seine ersten klinischen Erfahrungen und beschrieb dabei das Outcome von 15 durch ihn interventionell therapierten Patienten. 
Acht dieser Patienten hatten initial eine aSAB. Bei lediglich einem Patienten konnte ein zeitweise verschlechterter neurologischer Zustand durch das Verfahren registriert werden. Alle Aneurysmen waren zu mindestens 70\% okkludiert (Guglielmi et al. 1991). Seit 1995 ist das Coiling in Deutschland zugelassen und hat aufgrund seiner Effektivität und Minimal-Invansivität Einzug in die Leitlinien zur Behandlung von unrupturierten zerebralen Aneurysmen und der aSAB gehalten (DGN 2017b).

Der Verschluss eines aneurysmatragenden Blutgefäßes kann entweder nur proximal oder wie beim Trapping direkt proximal und distal des Aneurysmas geschehen. Vor allem bei fusiformen Riesenaneurysmen findet dieses Prozedere statt. Dies geschieht entweder chirurgisch mittels Clips oder interventionell via Coils oder absetzbarer Ballons. Voraussetzung für diese Therapie ist die gesicherte Perfusion des abhängigen Hirnareals. Dies kann anatomisch über ausgeprägte Kollateralkreisläufe oder durch die vorrausgegangene Anlage intrakranieller Bypässe gewährleistet sein. In Einzelfällen kann die Okklusion des Trägergefäßes, auch bei ischämischen Untergang des postaneurysmatischen Versorgungsgebietes in Erwägung gezogen werden. Ziel kann die Reduktion des Blutungsrisikos oder der Folgen der Raumforderung durch das Aneurysma sein. Das hohe Risiko dieses Eingriffs rechtfertigt eine invasive präoperative Diagnostik mittels Ballontestokklusion des betroffenen Areals. Eine transkranielle Doppler-Sonographie der zerebralen Gefäße und eine Perfusionsmessung vor und während der Ballontestokklusion empfehlen sich (Böcher-Schwarz et al. 2014).

\subsubsection{Die Neurochirurgische Behandlungsmethode, das Clipping}

Schon 1937 gelang die erste operative Klippung eines intrakraniellen Aneurysmas durch den Neurochirurgen Walter Dandy in Baltimore (USA) (Kretzer et al. 2010). Das Clipping ist ein neurochirurgisches Verfahren, bei dem die Operateure den Aneurysmasack mit einer kleinen Klammer von der Blutzirkulation abtrennen. Die Operateure müssen dabei darauf achten, dass es zum Erhalt des Trägergefäßes kommt. Liegt der Clip nicht richtig, kann es zu einer Stenosierung des Trägergefäßes oder einem Verbleiben eines Restaneurysmas kommen. Das Clipping galt bis zur Einführung endovaskulärer Methoden als Therapie der ersten Wahl. Die Operationstechniken und Materialien, wie die Clips selbst, konnten über die Jahre optimiert werden, sodass es heutzutage möglich ist, fast alle Aneurysmen offenchirurgisch zu erreichen und via Clip zu therapieren. Die Geometrie und Topographie der Aneurysmen kann das Clipping allerdings unmöglich machen (Böcher-Schwarz et al. 2014). 
Durch eine Ummantelung des Aneurysmasacks verspricht man sich einen Rupturschutz. Dieses Verfahren, also die Ummantelung des Aneurysmasacks mittels Eigen- oder Fremdgewebe, nennt sich Wrapping. Da das Aneurysma dabei nicht völlig ausgeschalten wird und es dennoch zu einer Größenzunahme und Ruptur des Aneurysmas kommen kann, wird die Effektivität des Verfahrens angezweifelt (Cossu et al. 1993).

\subsubsection{Die ISAT-Studie, ein Paradigmenwechsel in der Behandlung der aSAB}

Mit Einführung der endovaskulären Therapie kam früh die Frage auf, welches Verfahren das optimale sei. Kann diese Aussage generell beantwortet werden oder ist das eine Verfahren für eine Patientengruppe und das anderer Verfahren für eine andere Patientengruppe besser geeignet? Wie das Resultat, das Outcome, bei den verschiedenen Methoden ist, überprüfte 1997 die Universität Oxford in einer prospektiven, randomisierten Studien, der ISAT-Studie. An der International Subarachnoid Aneurysm Trial (ISAT) Studie nahmen weltweit 43 medizinische Zentren teil. Davon waren 39 aus Europa. Im Jahre 2002, als die Patientenakquise abgeschlossen wurde, waren 2143 Patienten mit aSAB in die Studie eingeschlossen. 7416 der während des Zeitraums innerhalb der Zentren behandelten Patienten wurden aufgrund der Einschlusskriterien und der Verletzung mindestens eines Kriteriums nicht mit in die Studie aufgenommen. Die Einschlusskriterien beinhalteten Folgendes:

- Das Vorhandensein einer SAB die jünger als 28 Tage war und anhand eines CT oder einer Lumbalpunktion bewiesen wurde.

- In der DSA oder einer CTA musste ein rupturiertes Aneurysma ursächlich erwiesen sein.

- Die Therapie muss während des Zeitpunkts der Randomisierung indiziert sein.

- Ein erfahrener Neurochirurg und Neuroradiologe mussten sich einig darüber werden, dass das rupturierte Aneurysma des Patienten für beide Verfahren (nämlich dem Clipping und Coiling) geeignet war.

- Zum Zeitpunkt der Randomisierung darf kein eindeutiger Vorteil für eines der Verfahren bestehen.

- Der Patient oder im Vertretungsfall seine Angehörigen sind nach Aufklärung einverstanden (Molyneux et al. 2002).

Nach einem Jahr publizierten Molyneux et al. im Jahre 2002 erste Ergebnisse. Bezogen auf die Mortalität und schwere Morbidität ergab sich ein signifikanter Vorteil für das 
Coiling. Beide Patientengruppen, sowohl die des Clippings als auch die des Coilings, haben in sehr kleiner Zahl nachgeblutet. Vergleicht man beide Gruppen untereinander, so ergibt sich bezüglich der Nachblutungen ein kleiner Vorteil für das Clipping. In absoluten Zahlen ergibt dieses: Zwei zu Null. Es kam demnach zu zwei Nachblutungen in der Coilinggruppe und keine in der Clippinggruppe (Molyneux et al. 2002).

Die Auswertung der ISAT-Studie stieß auf deutliche Kritik verschiedenster Gesellschaften der Neurochirurgie. Zu den Hauptkritikpunkten gehört, dass die Vorteile des Coilings nur einen kleinen Teil der Patienten mit aSAB betreffen. Grund hierfür ist, dass im Vorfeld 7416 der insgesamt 9559 Patienten aus der Studie ausgeschlossen wurden. Zudem sei die Langzeitprognose der endovaskulär versorgten Patienten weiterhin unklar. Sie verwiesen in diesem Zusammenhang auf die zwar kleine, aber dennoch höhere Nachblutungsrate gegenüber der geclippten Aneurysmen. US-Amerikaner stuften die ISATStudie als wenig vertrauenswürdig ein, da sehr wenige US-amerikanische Zentren eingeschlossen wurden. Sie zweifelten an der Erfahrung der nicht US-amerikanischen Neurochirurgen. Ein weiterer Hauptkritikpunkt war der zeitliche Abstand zwischen der Randomisierung und dem vollendeten Aneurysmaverschluss. Die Gruppe der via Clip versorgten Aneurysmen wies deutliche längere Zeiträume auf. Die daraus resultierende große Anzahl der präprozuduralen Nachblutungen dieser Gruppe würde das Endergebnis ebenfalls deutlich verzerren (Böcher-Schwarz et al. 2014). Auch die Deutsche Gesellschaft für Neurochirurgie positionierte sich sehr kritisch und verwies, bei einem schlechten Outcome von über 30\% der geclippten Patienten auf ein ausnehmend schlechtes Ergebnis der Clipping-Gruppe im Vergleich mit anderen Prospektiven Studien. Zudem sei das tatsächliche Outcome wesentlich von den Nachblutungen abhängig, zu denen nach einem Jahr noch keine konkreten Angaben gemacht werden können (Raabe et al. 2003).

Langzeitergebnisse wurden eingefordert, und im Jahre 2009 veröffentlichten Molyneux et al. Ergebnisse, die einer mittleren Nachbeobachtungszeit von 9 Jahren (6-14 Jahren) entsprach. Demnach war die Nachblutungsrate zwar weiterhin gering. Allerdings lag sie nun aber deutlich höher bei der Coiling-Gruppe. Ab dem sechsten Jahr kam es zudem zu keiner weiteren Nachblutung mehr. Die Mortalitätskurven glichen sich mit der Zeit an. Trotz häufiger Nachblutung ergab sich aber weiterhin ein Signifikant niedrigeres Mortalitätsrisiko als bei der Clipping-Gruppe (Molyneux et al. 2009). Das neuropsychologische Outcome wurde bei über 600 Patienten in 8 Zentren der ISAT-Studie differenziert getestet und bestätigte die Überlegenheit des Coilings gegenüber dem Clipping 
(Scott et al. 2010). Die deutsche Gesellschaft für Neurologie kritisiert noch in den heute gültigen Leitlinien (2016), dass ältere Patienten über 70 Jahre unterrepräsentiert seien. Gleiches gilt für jene Patienten, welche ein vertebrobasiläres Aneurysma aufweisen. Grund hierfür ist, dass bei diesen im Vorfeld eine endovaskuläre Therapie indizierter erschien und sie damit aufgrund der Verletzung eines Einschlusskriteriums aus der Studie ausgeschlossen wurden. Dasselbe gilt in umgekehrter Form für Aneurysmen der ACM, bei denen häufig die operative Versorgung initial als indizierter angesehen wurde (DGN 2017b).

McDougall et al. überprüften die Ergebnisse der ISAT-Studie mit einer eigens erstellten Studie am Barrow Neurological Institut in Phoenix, Arizona (USA). Die prospektive Studie randomisierte 472 Patienten in eine Clipping- und in eine Coiling-Gruppe. Um allen Kritikpunkten gerecht zu werden, wurden die Aneurysmen nur durch sehr erfahrene Ärzte verschlossen und das jeweils innerhalb von 24 Stunden nach Ereignis. Ein Drittel aller Patienten der Coiling-Gruppe wechselten aus Gründen, wie der Unmöglichkeit eines Coil-Verschlusses oder anderer medizinischer Gründen zur Clippping-Gruppe. Dieser Wechsel, cross over, wurde in die Statistik aufgenommen. So mussten 42\% (70/168) der Patienten für das Coiling eines Aneurysmas des anterioren Stromgebiets zum Clipping wechseln (Spetzler et al. 2015). Im Wesentlichen bestätigten sich die Ergebnisse der ISAT- Studie. Es ergab sich auch hier nach einem Jahr ein klarer Vorteil der endovaskulären Therapie, bezüglich einer schweren Morbidität und Mortalität (McDougall et al. 2012). Der Zwangswechsel innerhalb der Patientengruppe zeigte allerdings, dass es Situationen gibt, in der ein Verfahren dem anderen überlegen ist. Summa Summarum ergab sich ein Paradigmenwechsel aus der ISAT-Studie. Die Ergebnisse legten nahe, dass nun das Coiling als die Therapie der ersten Wahl angesehen werden muss. Da das Coiling ist nicht immer möglich ist, stellt das Clipping dann eine gute Therapie der zweiten Wahl dar. Auch andere Gründe, zum Beispiel das Vorhandensein offenchirurgisch zu behandelnder Komorbiditäten, wie eines intrazerebralen Hämatoms, lassen die gleichzeitige Klippung eines Aneurysmas innerhalb derselben Operation als sinnvoll erscheinen. Die Therapieplanung für die SAB sowie für das Vorhandensein unrupturierter Aneurysmen ohne vorrausgegangene $\mathrm{SAB}$ sollten generell in spezialisierten Zentren erfolgen. Jeder Patient sollte dementsprechend eine individuelle interdisziplinär erstellte Therapiestrategie erhalten (Böcher-Schwarz et al. 2014). 


\subsection{Das Management der SAB und ihre Komplikationen}

Die aSAB ist ein medizinischer Notfall und muss intensivmedizinisch beobachtet und behandelt werden. Die bisweilen weiterhin schlechte Prognose legitimiert eine invasive Diagnostik und Therapie. Prognosebestimmend sind vor allem der Schweregrad der SAB bei der Aufnahme und das Auftreten von intrakraniellen Infarkten (Böcher-Schwarz et al.2014). Zu Infarkten und Ischämien kommt es besonders häufig mit dem Auftreten von intrakraniellen Vasospasmen. Als Vaso- oder Angiospasmen bezeichnet man die krampfartige spastisch-kontraktile Verengung eines Blutgefäßes. Kommt es zu einer Minderdurchblutung des poststenotischen Versorgungsgebiets mit der Entwicklung neurologischer Defizite, so spricht man von einem symptomatischen Vasospasmus. Das zeitliche Maximum posthämorrhagischer Vasospasmen liegt zwischen dem dritten und dem vierzehnten Tag nach dem Ereignis und wird mittels Angiographie oder DopplerSonographie nachgewiesen. Es tritt bei mehr als einem Drittel der Patienten auf BöcherSchwarz et al. 2014). Vasoaktive Substanzen aus den zerplatzen Erythrozyten dienen als ätiologischer Erklärungsansatz. Kommt es zur Blutung in dem mit Liquor gefüllten Subarachnoidalraum, so platzen die Erythrozyten aufgrund der geringeren Tonizität des Liquors, mit der Folge, dass es zur Entleerung des Zellinhaltes in den Subarachnoidalraum kommt. Auch wenn derzeit kein genaues Verständnis vorliegt und es noch weiterer Forschungen bedarf, so gibt es eine klare Relation zwischen Blutmenge im Subarachnoidalraum und dem Auftreten von Vasospasmen (Fisher et al. 1980). Therapeutisch lassen sich medikamentöse und interventionelle Verfahren unterscheiden. Früher galt die sog. „Triple-H-Therapie“, bestehend aus Hypertension, Hypervolämie und Hämodilution, als anerkannte Therapie. Einen Nutzen dieser Therapie konnte trotz zahlreicher Studien nie belegt werden. Da sie sich stattdessen als nicht nebenwirkungsfrei erwiesen hat, kann, wenngleich die Triple-H-Therapie einem logischen Gedanken folgt, dieses Vorgehen nicht mehr empfohlen werden (Steiner et al. 2013). Gängige Praxis und Konsens besteht darin, den Blutdruck bei Patienten mit intrakraniellen Vasospasmen mindestens hochnormal zu halten, um ein Ischämie des poststenostischen Versorgungsgebiets zu vermeiden. Ein weiterer medikamentöser Behandlungsansatz besteht in der Gabe des Calciumkanalblockers Nimodipin. Nimodipin ist ein Calziumkanalblocker des DihydropyridinTyps und hemmt den spannungsabhängigen L-Typ-CA2+ Kanal an den glatten Muskelzellen. Ein herabgesetzter Muskeltonus mit konsekutiver Vasodilatation ist die Folge. Nimodipin ist von den vorhandenen Calciumkanalblockern der liquorgängigste und wird 
deshalb besonders für zerebrale Ereignisse verwendet. Die prophylaktische und therapeutische Gabe von Nimodipin bei intrakraniellen Vasospasmen ist umstritten. Die bislang einzig evidenzbasierte Primärprophylaxe ist die orale Gabe von 60mg Nimodipin alle 4 Stunden. Dies zeigte vor allem die große Literaturarbeit von Dorhout Mees et al., in der sie zwei Autoren unabhängig voneinander die bestehende Literatur prüfen ließen und dabei 16 verschiedene Studien verglichen (Dorhout Mees et al. 2007). Die täglichen kraniellen Dopplersonographien quantifizieren den Therapieerfolg. Für Patienten mit schweren intrakraniellen Vasospasmen kommt als Ultima Ratio die endovaskuläre Ballondilatation zum Einsatz. Die nicht ungefährliche mechanische Erweiterung des spastischen Gefäßes birgt das Risiko der Gefäßruptur und bedarf deshalb einer strengen Indikationsstellung, die individuell diskutiert werden sollte.

Im Liquorraum befindliches Blut birgt zusätzlich zu den Vasospasmen das Risiko der Liquorzirkulationsstörung. Die Blutkoagel verstopfen die Ausführungsgänge der Ventrikel und können die Granulationes arachnoidales tamponieren. Bei der posthämmorrhagischen Liquorzirkulationsstörung muss computertomographisch zwischen einem Verschlusshydrozephalus und der akuten Liquorzirkulationsstörung differenziert werden. Die Unterscheidung gelingt über die Betrachtung der Hirnventrikel. Eine isolierte Erweiterung der supratentoriell gelegenen Ventrikel spricht für einen akuten Verschlusshydrozephalus aufgrund der Verlegung der Ausführungsgänge des vierten Ventrikels. Im Gegensatz zur Liquorrückresorptionsstörung, bei der sich alle Ventrikel erweitert darstellen, kann dieser nicht lumbal drainiert werden (Böcher-Schwarz et al. 2014). Häufig indiziert die klinische Bewusstseinseintrübung, ausgelöst durch den steigenden Hirndruck, die Anlage einer offenen kranialen Liquordrainage.

\subsection{Die Computertomographie}

Alle in dieser Forschungsarbeit untersuchten bildgebenden Verfahren sind Röntgenstrahlung nutzende Verfahren. Godfrey Newbold Hounsfiel, geboren am 28. August 1919, war ein englischer Elektromechaniker. Für den Bau des ersten Computertomographen wurde er zusammen mit dem südafrikanisch-US-amerikanischen Physiker, Allen McLeod Cormack im Jahre 1979 mit dem Nobelpreis für Physiologie/Medizin beehrt. Man kann sagen, Hounsfield konstruierte die Hardware und nutze für die Software die Algorithmen Cormacks. 1971 gelang es eine Frau mit einer Hirnzyste computertomographisch zu durchleuchten. Hounsfields Scan dauerte neun Tage inklusive weiterer zwei Tage für die Rekonstruktion. Heute, einige Jahrzehnte später, dauert eine CT wenige 
Sekunden. Auch in anderen Bereichen wie Strahlungsmenge, Bildqualität etc. gibt es revolutionäre Weiterentwicklungen.

Prinzipiell bedient sich die CT der konventionellen Röntgenmethodik, also der Erstellung eines Bildes aufgrund unterschiedlicher Schwächungsgrade des Körpergewebes. Das Röntgenstrahlenbündel wird durch die unterschiedlichen Dichteverhältnisse des Körpers different absorbiert, sodass durch die Berechnung der einzelnen Projektionen ein Bild konstruiert werden kann. Die Software berechnet für jede Projektion die vom Detektor erfasste Strahlenschwächung, welche die Gewebedichte wiederspiegelt. Der gesamte Messdatensatz entspricht einer Matrix von 256x256, 512x512 oder zahlreicheren Werten und erzeugt ein zweidimensionales Schnittbild. Die Bildelemente (Pixel) entsprechen Volumenelementen (Voxel), welche in ihrer spezifischen Dicke festgelegt sind. Die Schichtdicke, die Matrixgröße und der Messfelddurchmesser bestimmen die Größe der Voxel. Jedem Matrixwert ist eine spezifische Graustufe zugeordnet. Herr Godfrey Newbold Hounsfield zu ehren wurde das Bezugssystem der Graustufen nach ihm benannt. Das Intervall der Hounsfield-Units (HU) reicht von -1000 bis +3000 , wobei reines Wasser die HU von Null besitzt. Eine Beurteilung des Schnittbildes wird durch die Komprimierung der Dichteskala (Fensterung) erleichtert. Gerade im Bereich der Neuroradiologie ist die genaue Wahl der Fensterung entscheidend, da das zu beurteilende Hirnparenchym nur geringe Unterschiede in der Dichte aufweist (Jansen O und Sartor K 2006) Zur Kontrastverstärkung unterschiedlich perfundierter Gewebe oder zur Beurteilung der Anatomie der Gefäße, zum Beispiel im Rahmen der Detektion von Aneurysmen, werden Kontrastmittel währende des Scannens intravenös verabreicht. Diese Kontrastmittel (KM) sind jodhaltig und nierengängig. Jodhaltige Kontrastmittel sind wasserlösliche Substanzen, die zu über 90\% renal ausgeschieden werden. Eine normale Nieren- und Schilddrüsenfunktion sollten unter Berücksichtigung der Indikation bei dem Patienten vorliegen, um Schaden durch das KM abzuwenden. Durch moderne Rekonstruktionsalgorithmen können individuelle Anpassungen, wie Bild- und Ortsauflösung, je nach Bedarf erreicht werden.

\subsection{Die Flachbilddetektor-Computertomographie (FDCT)}

1997 publizierten Fahrig et al. das Gelingen dreidimensionaler Angiographien mittels eines C-Bogen-Systems von diagnostischer Qualität (Fahrig et al. 1997). Ein mobiles System, welches während radiologischer Interventionen eine hohe Weichteildifferenzierung ermöglicht, wurde durch die FDCT real. Die konventionellen Radiographien verfügten 
nicht über geeignete Absorptionseffizienz und hatten einen zu kleinen Dynamikbereich. Andere Ideen, übliche CT-Systeme mobiler zu gestalten, sodass sie für interventionelle oder chirurgische Eingriffe zur Verfügung stehen könnten, wurden wegen mangelnder Praktikabilität und ökonomischen Gründen schnell verworfen. Funktionell entstehen bei der FDCT multiple zweidimensionale Röntgenaufnahmen durch die Rotation des C-Bogens um das durchleuchtete Objekt. Diese multiplen Röntgenaufnahmen können anschließend mittels spezieller CT-Algorithmen zu einem dreidimensionalen Datensatz rekonstruiert werden (Kamran et al. 2010).

\subsection{Die DSA}

Die digitale Subtraktionsangiographie (DSA) ist Goldstandard in der Diagnostik intrakranieller Aneurysmen. Es handelt sich dabei um ein besonderes Verfahren, bei dem durch Filtertechnik ein isoliertes Bild des zu begutachtenden Gefäßstromgebiets erzeugt wird. Primär wurde die DSA durch Mistretta et al. 1981 beschrieben. Sie erläuterten, wie man die Filtertechnik nutzen kann und welche Perspektiven sich für die zukünftige Radiologie bieten könnten (Mistretta et al. 1981). Nach dem Erstellen einer Leeraufnahme der zu untersuchenden Körperregion, der sog. Maske, wird, ohne die Projektion zu verändern ein weiteres Bild erstellt, welches während der Perfusion von zeitgleich, arteriell appliziertem Kontrastmittel entsteht. Die Maske wird nun durch die Software „subtrahiert“, sodass nur noch das Kontrastmittel-gefüllte Innenlumen des Gefäßsystems sichtbar ist. Vor allem Knochen und Weichteilstrukturen sind nun aus dem Bild gelöscht. Dies ermöglicht eine millimetergenaue Darstellung des Gefäßsystems und führt zu einer kontrollierten Katheterisierung durch eine sich wie eine Straßenkarte darstellende Gefäßstruktur. Dies ist im Bereich der Neuroradiologie, aufgrund der vielen Verzweigungen der Gefäße als auch der Vulnerabilität dieser Körperregion von besonderer Bedeutung.

Die DSA ist das Verfahren der ersten Wahl der interventionellen Radiologie. Dazu zählen die Versorgungen mit Stents bei arteriellen Stenosen, gleich ob in der Peripherie oder im Zentrum des Körpers, die Embolisation von Gefäßmalformationen oder das Coiling von Aneurysmen. Je nach Modell der DSA sind ca. 50 Bilder pro Sekunde möglich. Da die Projektionen in Echtzeit zur Verfügung stehen, sind damit auch dynamische Aufnahmen realisierbar.

Durch die hohe Bildauflösung ist eine detailgetreue Darstellung des Gefäßsystems samt Anomalien wie Stenosen, Kollateralkreisläufen oder Aneurysmen möglich. 


\subsection{Die Rotationsangiographie}

Die hohe Vernetzung der Hirngefäße verhindert innerhalb einer zweidimensionalen Darstellung häufig eine gute Sicht auf die Zielstruktur. Um Überlagerungen zu vermeiden, wurde der Wunsch nach einer Technik laut, welche eine Projektion der Zielstrukturen aus multiplen Projektionswinkeln ermöglicht. Anfang der 1970er Jahre entwickelten Voigt et al. anhand von Phantomstudien eine Technik für die Darstellung von intrakraniellen Aneurysmen. Bei dieser Phantomstudie rotierte eine Kameraeinheit mit einer Geschwindigkeit von $6^{\circ} /$ Sekunde um $180 \mathrm{Grad}$ um das Phantom (Voigt et al. 1975). Am Patienten fand diese Technik erstmals im Jahr 1983 Anwendung. Es diente ebenfalls zur Detektion intrakranieller Aneurysmen (Thron und Voigt 1983). Es war zum damaligen Zeitpunkt noch nicht möglich, Filtertechnik im Sinne einer Subtraktion zu verwenden. Die digital subtrahierte Rotationsangiographie (3D-DSA) sollte erst sechs Jahre später durch Schumacher et al. beschrieben werden (Schumacher et al. 1989). Diese innovative Technik ist heutzutage Goldstandard im Bereich der interventionellen Neuroradiologie. Ihr Hauptvorteil ist die dreidimensionale Darstellung der Zielstrukturen unabhängig von ihrer Komplexität sowie der überlagerungsfreien Betrachtung aus multiplen Blickwinkeln. 


\section{Fragestellung}

3.1 Vergleich der Messgenauigkeit der vier verschiedenen bildgebenden Verfahren (FDCTA, DSA, MDCTA und Rotationsangiographie) zu den bekannten Referenzgrößen der Aneurysmamodelle.

Die aus der Herstellung der Aneurysmamodelle bekannten Referenzgrößen werden mit den gemessenen Mittelwerten der einzelnen Verfahren für beide Aneurysmen verglichen. Das Verfahren soll ermittelt werden, dessen Messungen am nächsten an den Referenzgrößen liegt. Ferner sollen natürlich die Verfahren, die große Abweichungen zu den Referenzgrößen aufweisen, untersucht werden. Durch diese Erhebung lässt sich eine erste Hierarchie der Verfahren bzgl. ihrer Messgenauigkeit erstellen.

\subsection{Vergleich der Messgenauigkeit zwischen dem aus der Studie hervorgegangenen Goldstandard und den anderen bildgebenden Verfahren.}

Initial werden alle verschiedenen Verfahren mit den Referenzgrößen verglichen. Das Verfahren wird als Goldstandard festgelegt, welchen aus der ersten statistischen Auswertung als das genauste hervorgeht. In der zweiten Fragestellung untersuchen wir diese These mit einem genauen Vergleich der einzelnen bildgebenden Verfahren (MDCTA, FDCTA und Rotationsangiographie) in Bezug auf die Messgenauigkeit des neuen Goldstandards.

\subsection{Die Rolle der Erfahrung der Untersucher bei der Ausmessung und Nutzung der verschieden bildgebenden Verfahren.}

Als Untersucher wurden gezielt drei Neuroradiologen mit unterschiedlicher Erfahrung ausgewählt. Einer der drei ist eher als unerfahren, einer als mittel und einer als erfahren, in Abhängigkeit von ihrem radiologischen Ausbildungsstand, einzustufen. Wir untersuchen, ob die Erfahrung einen Einfluss auf die Messgenauigkeit hat. Wir vergleichen dafür die Messergebnisse der einzelnen Untersucher bei allen bildgebenden Verfahren, bezogen auf die Referenzgrößen. Es wäre denkbar, dass die unerfahrenen Untersucher mit einem Verfahren genauer messen als mit anderen, potenziell sensitiveren Verfahren. 


\section{Material und Methodik}

\subsection{Versuchsaufbau}

Für den Versuch wurde ein Phantom geschaffen. Des Weiteren standen den Untersuchern zwei verschiedene Aneurysmamodelle zur Verfügung. Die Aneurysmamodelle sind 3D-Drucke aus Plastik, welche die Firma Phenox (Phenox GmbH, Bochum, Deutschland) aus Patientendatensätzen mittels eines Form-2 3D-Drucker (Formlabs, Somerville, Massachusetts) anfertigte und freundlicherweise spendete. Die originalen Datensätze wurden daher als Referenzdaten herangezogen. Die Modelle, ausgestattet mit drei und vier Anschlüssen, wurden mit handelsüblichen, $75 \mathrm{~cm}$ langen Verlängerungen des Typs Heidelberger (B. Braun Melsungen, Deutschland) bestückt. Anschließend wurden sie mit 0,9\% iger NaCl-Lösung (B. Braun Melsungen, Deutschland) gespült und entlüftet. Durch das Offenhalten eines Anschlusses und das Spülen durch einen anderen Anschluss konnte ein Fluss innerhalb des Aneurysmamodells generiert werden. Die Spülung und die spätere Gabe des Kontrastmittelgemisches erfolgten durch eine 50-ml-Perfusorspritze (Syringe, B. Braun Melsungen, Deutschland). Für den Versuch wurde das jodhaltige nicht-ionisierende Kontrastmittel Imeron genutzt (Imeron 350, Bracco ALTANA Pharma, Konstanz, Deutschland). Da Wasser und Hirnparenchym eine ähnliche Dichte besitzen, wurden Gel-Kühlkompressen (Reusable Cold Pack, Mueller Sports Medicine, Prairie du Sac, WI, USA) als Ummantelung des Modells verwendet. Zur besseren Simulation eines klinischen Aufbaus wurden die Modelle innerhalb eines anatomischen Schädels platziert. Die abnehmbare Schädelkalotte wurde während der Bilderserien wieder anatomisch platziert. Ein ähnlicher Versuchsaufbau mit Gelkompressen als Hirnparenchym wurde bereits von vorhergehenden Forschungsgruppen verwendet und belegt die Repräsentativität dieser Methodik (Psychogios et al. 2013). 


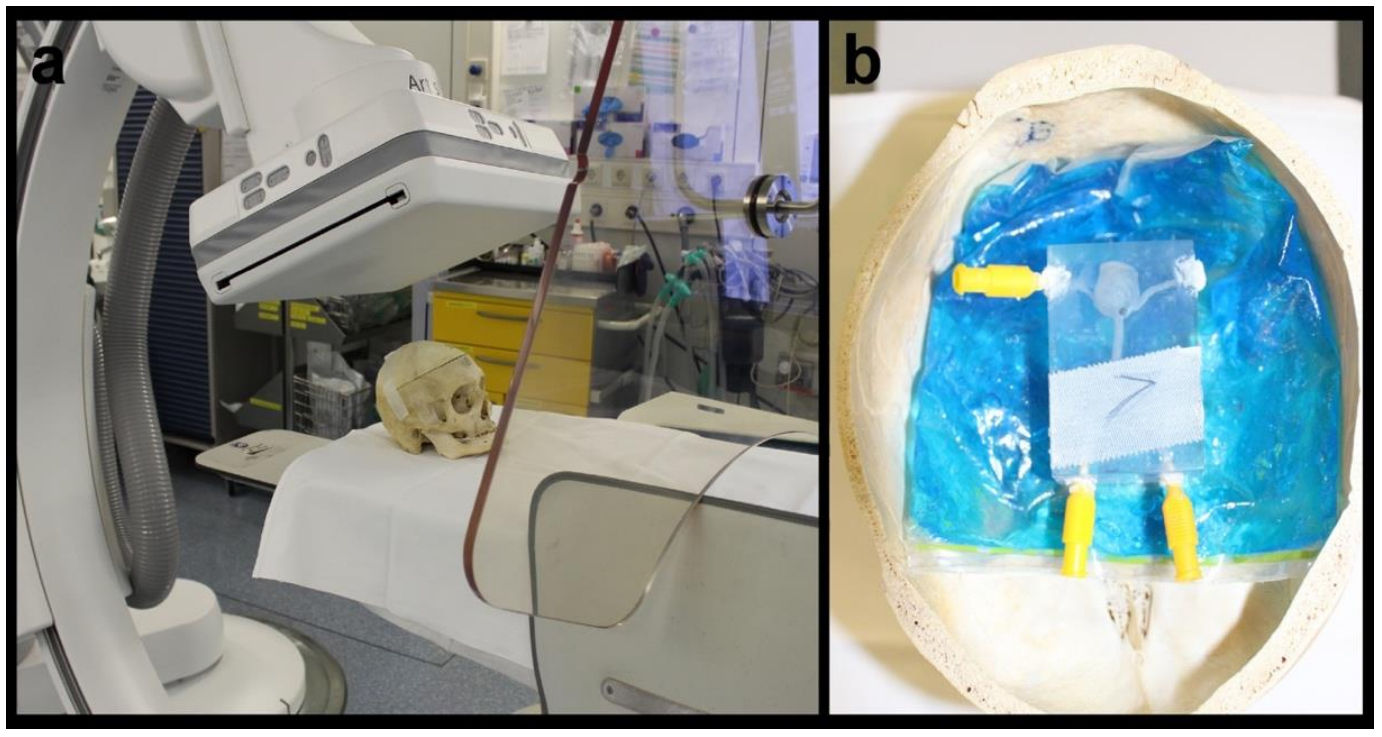

Abbildung 3: Foto des Versuchaufbaus; Das Aneurysmamodell in einer menschlichen Schädelkalotte auf dem Angiographietisch des biplane Artis Q

\subsection{Die Aneurysmamodelle}

Beide Aneurysmamodelle waren 3D-Drucke eines Patientendatensatzes, welche als Referenzdaten angesehen wurden. Es wurde darauf geachtet, dass beide unterschiedlicher Größe und Konfiguration waren. So konnte schon makroskopisch ein kleines von einem größeren Aneurysma unterschieden werden. Gemessen wurden jeweils die Höhe, der Hals und die Breite des Aneurysmas.

Referenzwerte der zwei Aneurysmamodelle:

\section{Aneurysmamodell 1:}

Hals: $7,5 \mathrm{~mm}$

Breite: $10,9 \mathrm{~mm}$

Höhe: 11,0 mm

\section{Aneurysmamodell 2:}

Hals: 3,2 mm

Breite: 3,4 mm

Höhe: $3,7 \mathrm{~mm}$ 


\subsection{Die Verwendeten CT-Geräte}

Die DSA und FDCTA Bilder wurden mittels biplanen Flachdetektor-Angiographen (Artis Q; Siemens; Erlangen, Deutschland) erstellt. Für die FDCTA wurde eine kommerziell verfügbare Sequenz mit einer Rotationszeit von 10 Sekunden, 70 kV, 1,2 $\mu \mathrm{Gy} /$ Projektion, $200^{\circ}$ Winkelbreite und $0,8^{\circ}$ Winkelschritt genutzt (Dyna-CT Clear; Siemens). Der

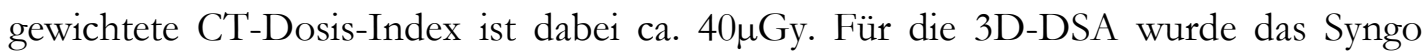
Dyna-CT-3D-Setup (Siemens) mit einer Rotationszeit von 5 Sekunden, 70 kV, 0,36 $\mu \mathrm{Gy} /$ Projektion, $0,8^{\circ}$ Winkelschritt und einen gewichteten CT-Dosis-Index von ca. 9 $\mu \mathrm{Gy}$ genutzt. Die MDCTA Bilder wurden mit eine 128 Zeilen CT-Scanner (Somatom Definition AS+; Siemens) aufgenommen. Dieser hat folgende Parameter: 128-Zeilen mit Kollision bei 0,6 mm; Pitch von 0,6, Parameter der Bildaquise bei 120 kV/140. Die Rohdaten wurden mit einem scharfen Rekonstruktionskern (nur für die MDCTA) und einer Betrachtungsbreiten (Scheibendicke) von $0.6 \mathrm{~mm}$ in einen Volumendatensatz umgewandelt.

Die Aufbereitung der Rohdaten der MDCTA und FDCTA sowie der VRT- und MPRBilder wurde auf einer Syngo-Workstation durchgeführt, indem wir die 3D oder 4DTool verwendeten (Syngo X Workplace; Siemens). Die Rekonstruktionen der 3D-DSAVRT und FDCTA-VRT-Bilder wurden mit einem weichen Kernel und kleiner VOI, die der MPR aus einem normalen Kernel und kleiner VOI erstellt.

Tabelle 6: Untersuchungsparameter

\begin{tabular}{|l|l|l|l|l|}
\hline & MDCTA & FDCTA & 3D-DSA & DSA \\
\hline FOV $(\mathrm{cm})$ & $10 \times 10$ & $10 \times 10$ & $10 \times 10$ & $10 \times 10$ \\
\hline Matrix size & $512 \times 512$ & $512 \times 512$ & $512 \times 512$ & $1024 \times 1024$ \\
\hline $\begin{array}{l}\text { In-plane resoluti- } \\
\text { ons (mm) }\end{array}$ & 0,2 & 0,2 & 0,29 & 0,15 \\
\hline $\begin{array}{l}\text { Cumilative dose } \\
\text { (mGy) }\end{array}$ & 30 & 40 & 9 & 53 \\
\hline
\end{tabular}




\subsection{Verwendete Abkürzungen und untersuchte Verfahren und Rekonstruktionen im Überblick}

MD-CTA-VRT $\rightarrow$ MD-CTA Bilder aus VRT-Rekonstruktionen

MD-CTA-MPR $\rightarrow$ MD-CTA Bilder aus MPR-Rekonstruktionen

FD-CTA-VRT $\rightarrow$ FD-CTA Bilder aus VRT-Rekonstruktionen

FD-CTA-MPR $\rightarrow$ FD-CTA Bilder aus MPR-Rekonstruktionen

$D S A \_$long $\rightarrow$ konventionelle 2D-DSA-Bilder mit langen Projekt-zu-Detektorabstand

$D S A \_$middle $\rightarrow$ konventionelle 2D-DSA-Bilder mit mittlerem Projekt-zu-Detektorabstand

$D S A \_n e a r \rightarrow$ konventionelle 2D-DSA-Bilder mit nahem Projekt-zu-Detektorabstand $D S A \_r o t a t i o n \_M P R \rightarrow$ konventionelle 3D-DSA-Bilder aus MPR-Rekonstruktionen $D S A \_r o t a t i o n \_V R T \rightarrow$ konventionelle 3D-DSA-Bilder aus VRT-Rekonstruktionen $D S A \_r o t a t i o n \_l o n g ~ \rightarrow$ 2D-DSA-Bilder, bei denen durch eine zuvor gelaufene 3D-DSA Bildgebung, ein optimaler Projektionswinkel erstellt wurde mit langem Objekt-zu-Detektorabstand

$D S A \_r o t a t i o n \_m i d d l e ~ \rightarrow 2 D-D S A$ Bilder, bei denen durch eine zuvor gelaufene 3D-DSA Bildgebung ein optimaler Projektionswinkel erstellt wurde mit mittlerem Objekt-zu-Detektorabstand

$D S A \_r o t a t i o n \_n e a r \rightarrow 2 \mathrm{D}-\mathrm{DSA}$ Bilder, bei denen durch eine zuvor gelaufene 3D-DSA Bildgebung ein optimaler Projektionswinkel erstellt wurde mit kurzem Objekt-zu-Detektorabstand

\subsection{Bildgebung und Datenakquise}

Beide Modelle wurden durch die verschiedenen Verfahren (nämlich MDCTA, FDCTA, 2D-DSA, 3D-DSA) in fünf verschiedenen Ausgangspositionen untersucht. Zusätzlich wurde in einer Untersuchungsreihe die Rotationsangiographie mit üblichen DSA Serien kombiniert.

Die 2D-DSA-Serien wurden mit drei verschiedenen Abständen (lang, mittel, nah) zum Detektor durchgeführt. Dies wurde einmal mit einem zufällig entstandenen Projektions- 
winkel und einmal mit nach durchlaufener 3D-DSA erstelltem optimalem Projektionswinkel realisiert. Insgesamt kamen wir auf eine Akquise von 90 Bildern. Durch die verschiedenen Rekonstruktionen in Volumen-Rendering-Technik (VRT) und multiplanarer Rekonstruktion (MPR) entstanden 120 Datensätze, bestehend aus den jeweiligen Messungen der Radiologen. Jedes akquirierte Bild wurde auf der Syngo Workstation (syngo X Workplace, Siemens) von drei Radiologen mit unterschiedlichem Erfahrungsniveau in der Aneurysmadiagnostik und -behandlung (7 Jahre, 5 Jahre, 3 Jahre) individuell analysiert und ausgemessen. Den Radiologen waren die Datensätze der Modelle unbekannt und sie waren nicht an der Akquise beteiligt. Sie wurden gebeten, anhand der erstellten Bilder das Aneurysma bzgl. des Aneurysmahalses, der maximalen Höhe und Breite auszumessen. Die Messergebnisse wurden ihrer Modellnummer entsprechend nach Untersuchungsmethode, Halsgröße, Höhe und Breite in eine Tabelle eingetragen. Der Mittelwert der einzelnen Untersucher wurden mit den bekannten Daten der Aneurysmamodelle verglichen. Für die 2D-DSA Serien, die FDCTA und MDCTA wurde jeweils ein NaClzu-Kontrastmittel-Verhältnis von 1:10 benutzt. Damit wurde ein authentischer Dichterwertunterschied zwischen dem kontrastmittelgefüllten Aneurysma und seiner Umgebung erreicht. Dieser entspricht ca. 300-400 Hounsfield Units (HU). Für die 3D-DSA wurde ein Verdünnungsverhältnis von 1:2 benötigt, um authentische Dichtewertunterschiede zu erreichen. Die Applikation des Kontrastmittelgemisches wurde durch eine handelsübliche Spritze (B. Braun Melsungen, Deutschland) realisiert.

\subsection{Die statistische Auswertung}

Es wurden Bland-Altman-Plots für jedes Verfahren erstellt. Dadurch konnten die gemittelten Werte dargestellt und aufzeigt werden. Das Verfahren, welches die geringste Abweichung zu den bekannten Größen des Aneurysmamodells hatte, wurde als „Goldstandard“ definiert. Um die verschiedenen Verfahren vergleichen zu können, wurden BlandAltman-Plots für alle Verfahren im Vergleich zum Goldstandard erzeugt. Dafür wurden die Unterschiede beider Verfahren gegen den Mittelwert beider Verfahren aufgetragen. Für die statistische Auswertung wurde das arithmetische Mittel, das Konfidenzintervall des arithmetischen Mittels (95\% CI) und die obere und untere Grenze (+1,96 SD) kalkuliert. Die Untersucher-Reliabilität wurde mittels intraclass correlation coefficient (ICC) bestimmt, wobei ein ICC > 0,8 als fast perfekte Übereinstimmung anzusehen ist. Die statistische Auswertung erfolgte mit dem Programm MedCalc statistical package (MedCalc, Mariakerke, Belgium). 


\section{Ergebnisse}

Nach Auswertung aller Verfahren und Rekonstruktionen erzielte die DSA nach Bestimmung des optimalen Projektionswinkels durch eine vorangegangene Rotationsangiographie und zusätzlich gering eingestellten Objekt-zu-Detektorabstand die den Referenzwerten am nächsten kommenden Messwerte. Wir haben dieses Verfahren als „DSA_rotation_near" abgekürzt. Statistisch ausgedrückt hatte die „DSA_rotation_near“ die niedrigste Abweichung absolut und nach Mittelung der Werte (smallest deviation SD) zu der bekannten Aneurysmagröße.

Die Messungen der drei Radiologen hatten im Mittel eine Differenz von -0,07 mm bei einer SD von 0,61 für Aneurysma eins. Für Aneurysma zwei ergab sich eine mittlere Differenz von $0,12 \mathrm{~mm}$ und eine SD von 0,25.

Ähnliche Ergebnisse erzielte die DSA nach Rotationsangiographie bei langen oder mittlerem Objekt-zu-Detektorabstand, wohingegen FDCT, MDCT oder auch die DSA unabhängig von der MPR oder VRT Einstellung größere gemittelte Unterschiede aufzeigten. Die Bland-Altman-Plots zeigen, dass die ausmessenden Radiologen mit den meisten Verfahren die Aneurysmagröße überschätzten. Gemäß dem oben beschriebenen methodischen Vorgehen wurde nun die DSA_rotation_near als Goldstandard definiert und alle anderen Verfahren damit verglichen. Der geringste Unterschied zeigte sich bei dem Vergleich der DSA-Serien nach optimiertem Projektionswinkel durch eine Rotationsangiographie, aber mit verschiedenen Objekt-zu-Detektorabstand. Dem entsprechend wurde

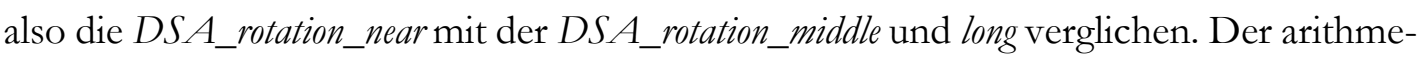
tische gemittelte Unterschied zwischen $\operatorname{der} D S A \_r o t a t i o n \_n e a r$ und $\operatorname{der} D S A \_r o t a t i o n \_m i-$ ddle (Mittlerer Objekt-zu-Detektorabstand nach optimaler Winkeleinstellung durch die Rotationsangiographie) war 0,02 (95\% CI: -0,08 zu 0,11; untere Grenze: -0,8851, 95\% CI: -1,06 - 0,72; obere Grenze: 0,92; 95\% CI: 0,75-1,08). Der arithmetisch gemittelte Unterschied aus dem Vergleich der DSA_rotation_near mit der DSA_rotation_long lag bei 0,09 (95\% CI: -0,04-0,22, untere Grenze -1,13; 95\% CI: -1,36 - 0,91; obere Grenze 1,31; 95\% CI: 1,09-1,54). Nach Gegenüberstellung des Goldstandards mit den konventioneller 2D-DSA-Serien ohne zuvor optimierten Projektionswinkel lag der geringste arithmetisch gemittelte Unterschied bei der 2D-DAS-Serie mit dem längsten Objekt-zu-Detektorabstand und war bei -0,48 (95\% CI: -0,63 - 0,33; untere Grenze -1,90, 95\% CI: -2,16 - 1.64, obere Grenze 0,94 95\% CI: 0,68 - 1,20). Für die 2D-DSA mit mittlerem Objekt-zuDetektorabstand war der gemittelte Unterschied bei -0,51 (95\% CI: -0,67-0,36; unterer 
Grenze $-1,98,95 \%$ CI: $-2,25--1,71$; obere Grenze 0,95, 95\% CI: 0,68-1,22) und $-0,58$ (95\% CI: -0,75- 0,41; untere Grenze -2,44; 95\% CI: -2,44 - 1,96, obere Grenze: 0,99; 95\% CI: 0,70-1,28) für die 2D-DSA mit nahem Objekt-zu-Detektorabstand. Der Vergleich des Goldstandards „DSA_rotation_near“ zwischen 2D-DSA-Bildern in MPR und VRT ermittelt nach durchgeführter Rotationsangiographie zeigte, dass die geringere Abweichung bei der DSA_rotation_MPR lag, nämlich -0,72 (95\% CI: -0,98 - 0,46, untere Grenze: -3,19--2,74; obere Grenze 1,75; 95\% CI: 1,30-2,20) gegenüber -0,86 (95\% CI: 1,03--0,69; untere Grenze: 0,77; 95\% CI: 0,70-1,07) der VRT-Bilder nach erfolgter Rotationsangiographie. Der Vergleich mit den angiographischen Bildern der MDCTA zum Goldstandard zeigte eine mittlere Abweichung von -0,84 (95\% CI: -1,06- -0,62; untere Grenze -2,89; 95\% CI: -3,27- -2,52; obere Grenze 1,22; 95\% CI: 0,85-1,60). Ebenfalls wurden VRT-Rekonstruktionen aus der Bildakquise der MDCTA mit der DSA_rotation_near verglichen. Dies resultierte in einer gemittelten Abweichung von -1,34 (95\% CI: 1,56- -1,13; untere Grenze -3,37 95\% CI: -3,74- -2,99; obere Grenze 0,68; 95\% CI: 0,311,05). Die Messwerte der FDCTA lag nach VRT-Rekonstruktion bei einer gemittelten Abweichung von -0,67 (95\% CI: -0,86- -0,49; untere Grenze -2,37; 95\% CI: -2,68- -2,06; obere Grenze 1,02; 95\% CI: 0,71 - 1,33) und mit MPR-Rekonstruktion bei -0,64 (95\% CI: 1,40-0.91--0,37; untere Grenze: -3,14; 95\% CI: -3,60 - -2,68; oberer Grenze 1,86; $95 \%$ CI: $1,40-2,32)$. 


\subsection{Graphische Darstellung der Messergebnisse}

Nachfolgend sind die Ergebnisse der verschiedenen Vergleichsuntersuchungen in BlandAltman-Plots graphisch dargestellt.

\section{DSA rotation near $-F D C T A$}

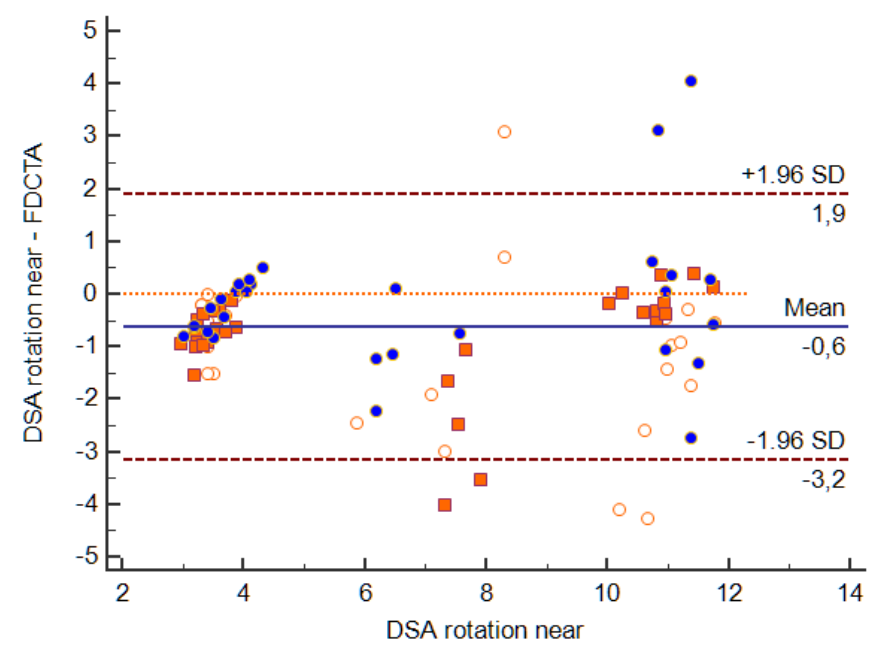

Abbildung 4: Graphische Darstellung in Bland-Altman-Plots der DSA_rotation_near gegen die FDCTA

\section{DSA rotation near - FDCTA VRT}

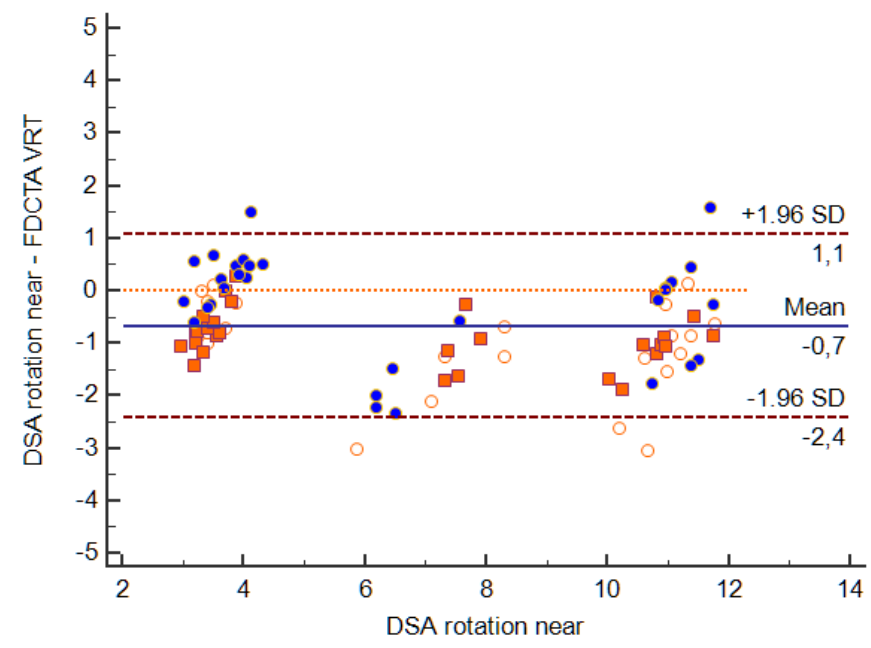

Abbildung 5: Graphische Darstellung in Bland-Altman-Plots der DSA_rotation_neargegen die FDCTA_VRT 
$\underline{D S A}$ rotation near $-M D C T A$

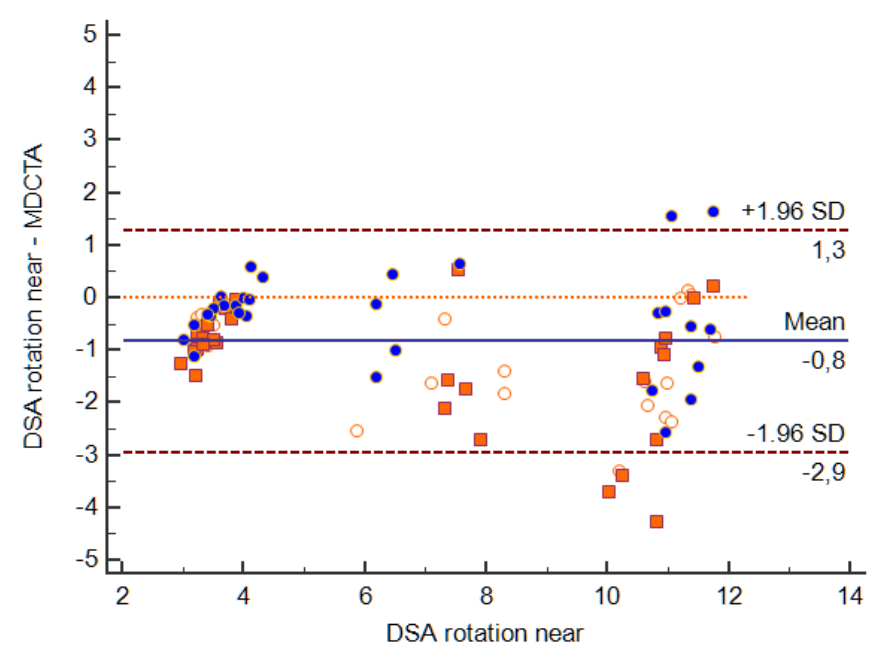

Abbildung 6: Graphische Darstellung in Bland-Altman-Plots der DSA_rotation_near gegen die MDCTA

\section{$\underline{D S A}$ rotation near $-M D C T A V R T$}

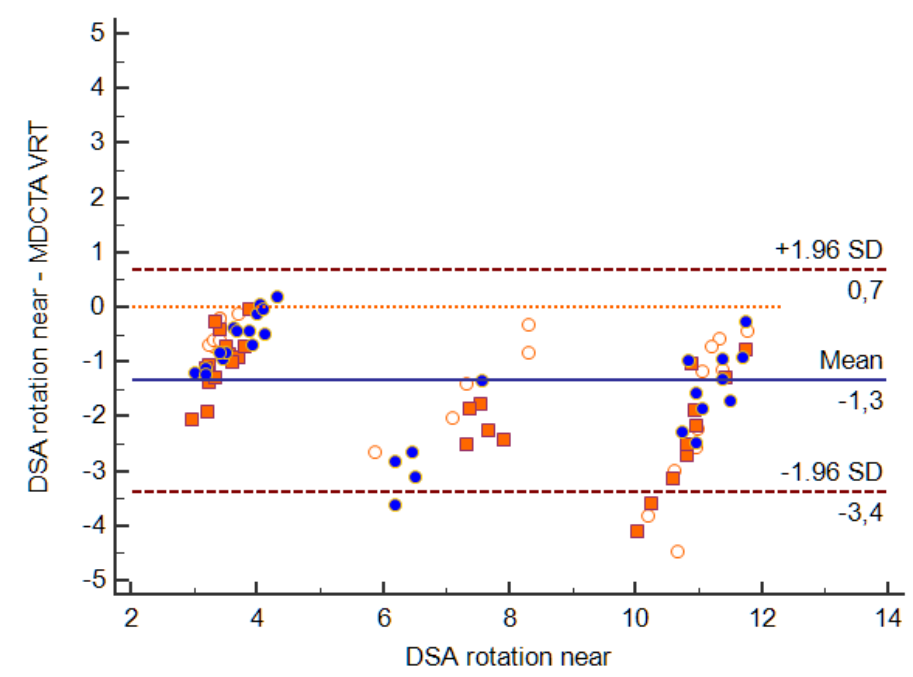

Abbildung 7: Graphische Darstellung in Bland-Altman-Plots der DSA_rotation_near gegen die MDCTA_VRT 


\section{DSA rotation near-DSA long}

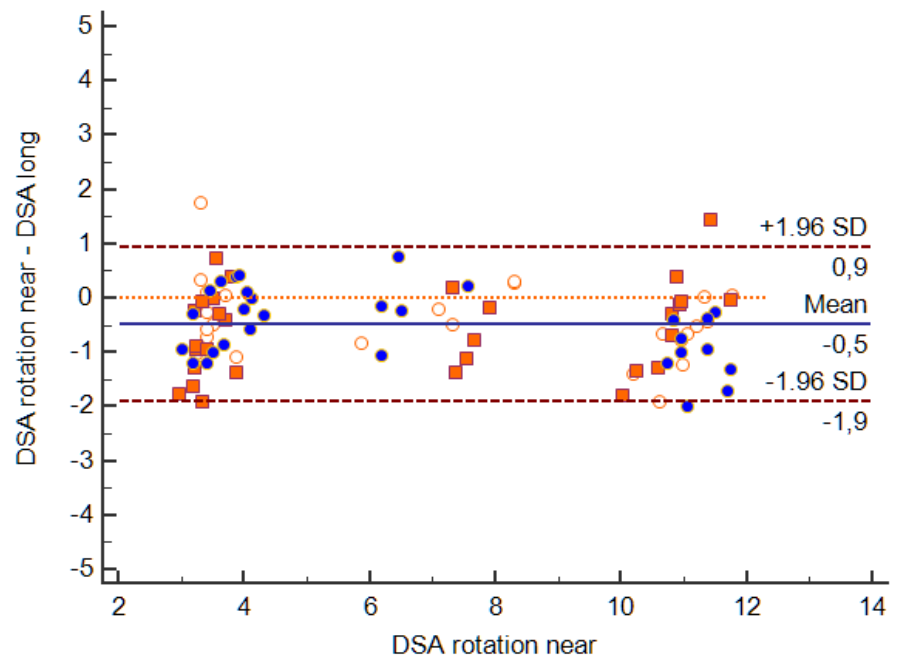

Abbildung 8: Graphische Darstellung in Bland-Altman-Plots der DSA_rotation_near gegen die DSA_long

\section{$\underline{D S A}$ rotation near $-D S A$ middle}

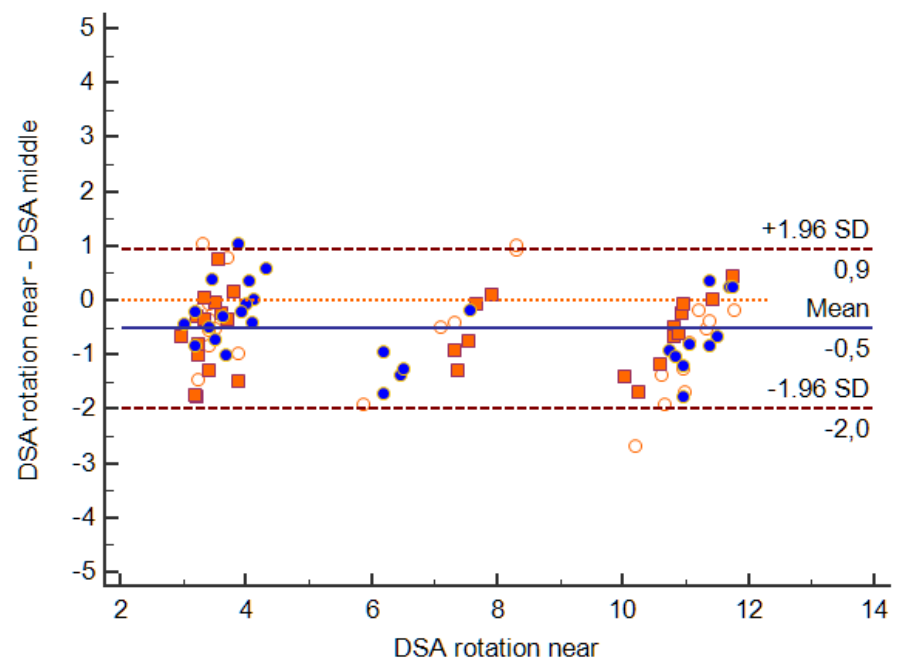

Abbildung 9: Graphische Darstellung in Bland-Altman-Plots der DSA_rotation_near gegen die $D S A \_$middle 
$D S A$ rotation near $-D S A$ near

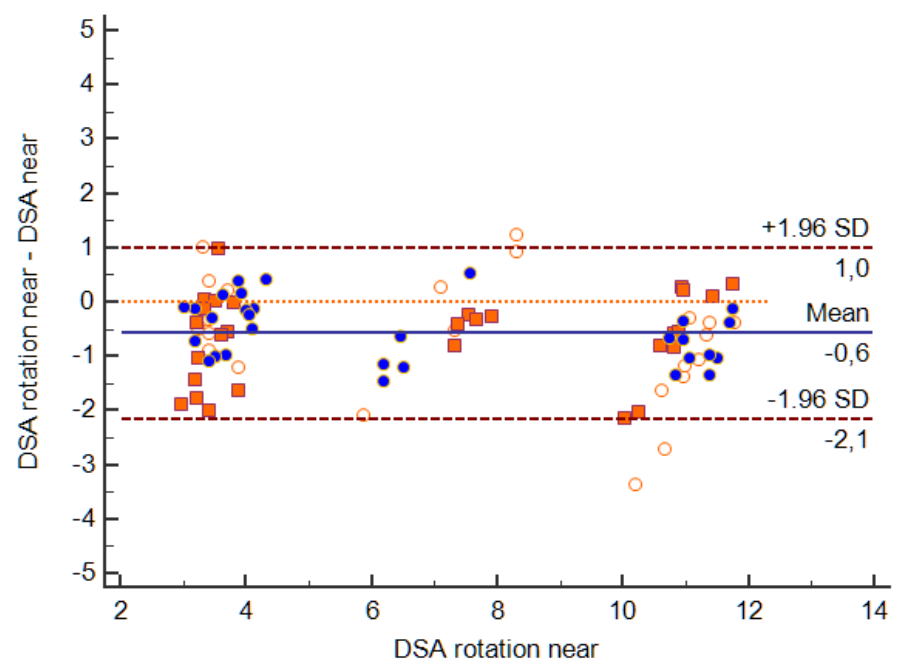

Abbildung 10: Graphische Darstellung in Bland-Altman-Plots der DSA_rotation_near gegen die DSA_near

\section{DSA rotation near - DSA rotation long}

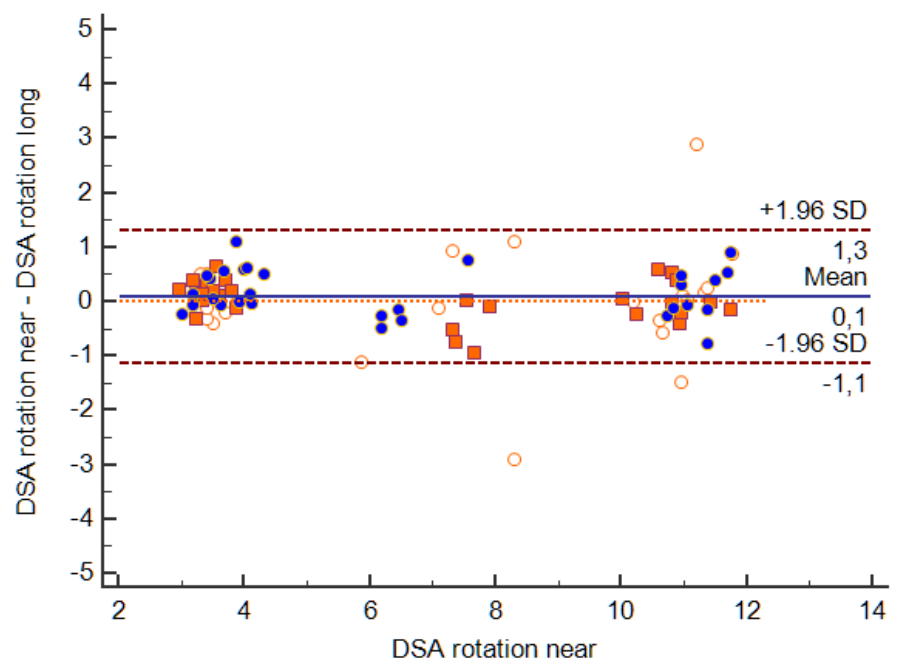

Abbildung 11: Graphische Darstellung in Bland-Altman-Plots der DSA_rotation_near gegen die DSA_rotation_long 
$\underline{D S A}$ rotation near $-D S A$ rotation middle

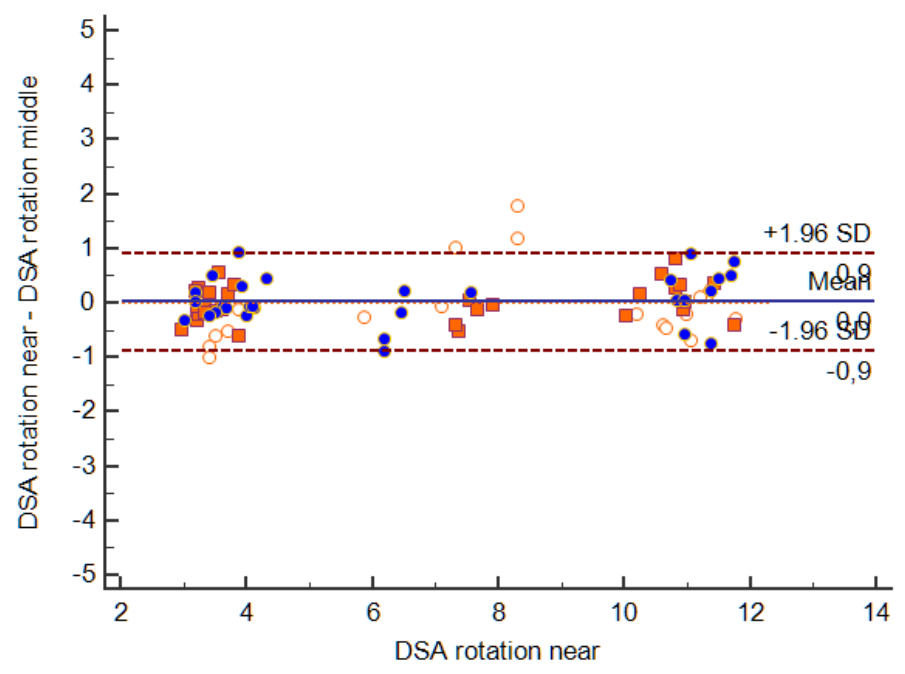

Abbildung 12: Graphische Darstellung in Bland-Altman-Plots der DSA_rotation_near gegen die DSA_rotation_middle

\section{$\underline{D S A}$ rotation near - Rotation $V \mathrm{RT}$}

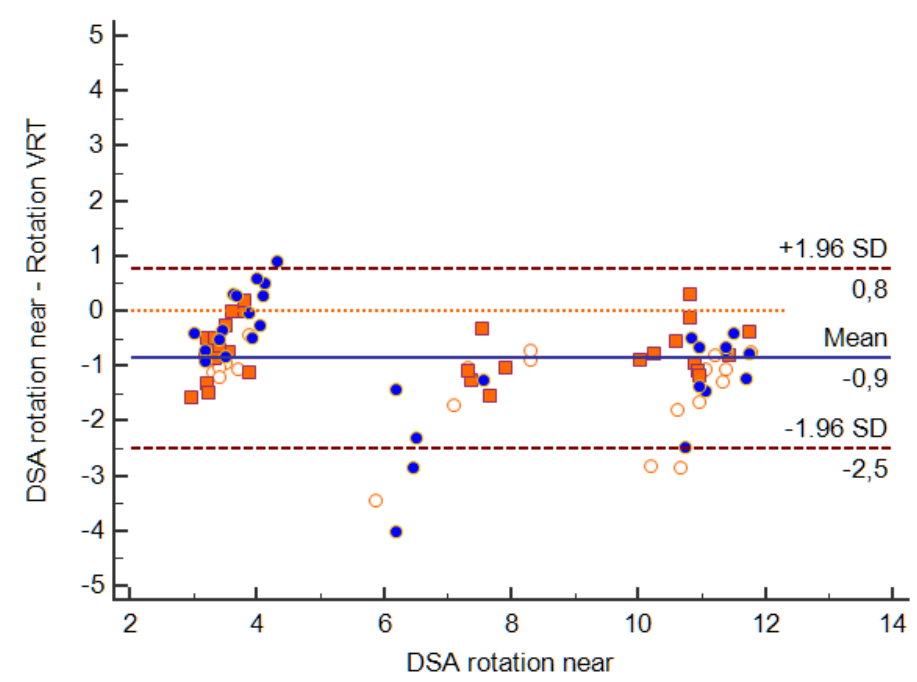

Abbildung 13: Graphische Darstellung in Bland-Altman-Plots der DSA_rotation_near gegen die Rotation_VRT 
DSA rotation near-Rotation $M P R$

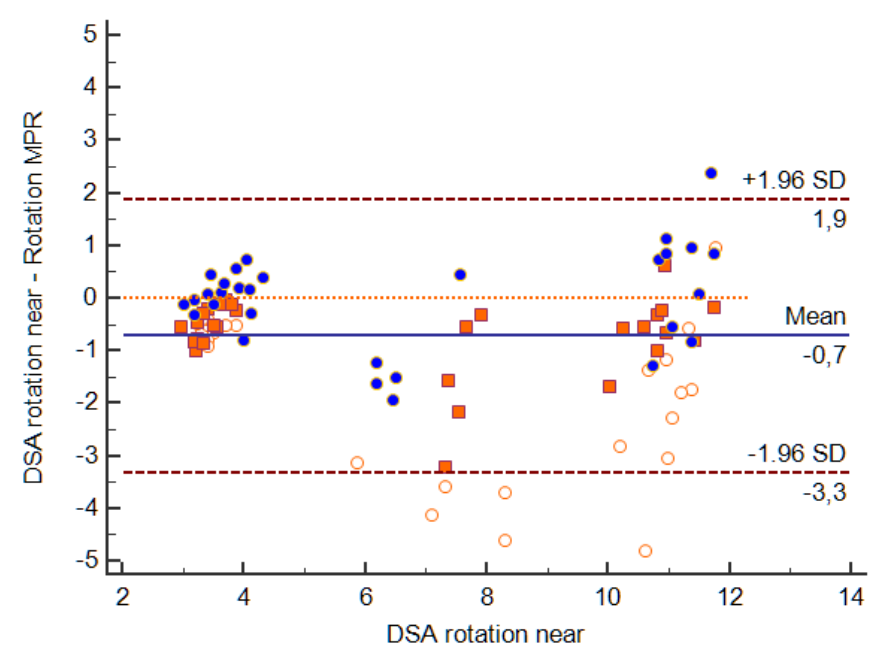

Abbildung 14: Graphische Darstellung in Bland-Altman-Plots der DSA_rotation_near gegen die Rotation_MPR 


\subsection{Tabellarische Darstellung der Messergebnisse}

Im Folgenden sind die Messergebnisse tabellarisch dargestellt.

Tabelle 7: Aneurysma I. mittlere Abweichung zu den Referenzgrößen (alle drei Dimensionen kombiniert)

\begin{tabular}{|c|c|c|c|c|c|}
\hline $\begin{array}{l}\text { Statistische } \\
\text { Daten/Verfahren }\end{array}$ & $\begin{array}{l}\text { Mittelwert } \\
(\mathrm{mm})\end{array}$ & $\mathrm{SD}$ & $\begin{array}{l}\text { SE of } \\
\text { mean }\end{array}$ & $\begin{array}{l}\text { Untere } \\
\text { Grenze } \\
95 \% \mathrm{CI}\end{array}$ & $\begin{array}{l}\text { Obere } \\
\text { Grenze } \\
95 \% \mathrm{CI}\end{array}$ \\
\hline FD-CTA MPR & 0,75 & 1,64 & 0,24 & 0,26 & 1,24 \\
\hline FD-CTA VRT & 0,99 & 0,69 & 0,10 & 0,78 & 1,20 \\
\hline MD-CTA MPR & 1,15 & 1,26 & 0,19 & 0,77 & 1,52 \\
\hline MD-CTA VRT & 1,91 & 0,69 & 0,10 & 1,70 & 2,11 \\
\hline$D S A$ long & 0,50 & 0,84 & 0,13 & 0,25 & 0,75 \\
\hline DSA_middle & 0,65 & 0,52 & 0,08 & 0,49 & 0,80 \\
\hline$D S A \_n e a r$ & 0,66 & 0,71 & 0,11 & 0,45 & 0,87 \\
\hline$D S A$ _rotation_MPR & 1,10 & 1,55 & 0,23 & 0,63 & 1,56 \\
\hline$D S A$ rotation_V $\mathrm{R} T$ & 1,19 & 0,69 & 0,10 & 0,98 & 1,40 \\
\hline$D S A \_r o t a t i o n \_l o n g$ & $-0,05$ & 1,71 & 0,25 & $-0,56$ & 0,47 \\
\hline DSA_rotation_middle & $-0,15$ & 0,64 & 0,09 & $-0,34$ & 0,04 \\
\hline$D S A$ $A$ rotation_near & $-0,07$ & 0,61 & 0,09 & $-0,25$ & 0,18 \\
\hline
\end{tabular}


Tabelle 8: Aneurysma II. mittlere Abweichung zu den Referenzgrößen (alle drei Dimensionen kombiniert)

\begin{tabular}{|l|l|l|l|l|l|}
\hline $\begin{array}{l}\text { Statistische } \\
\text { Daten/Verfahren }\end{array}$ & $\begin{array}{l}\text { Mittelwert } \\
(\mathrm{mm})\end{array}$ & $\mathrm{SD}$ & SE of mean & $\begin{array}{l}\text { Untere } \\
\text { Grenze }\end{array}$ & $\begin{array}{l}\text { Obere } \\
\text { Grenze } \\
95 \% \mathrm{CI}\end{array}$ \\
\hline FD-CTA MPR & 0,62 & 0,60 & 0,09 & 0,44 & 0,79 \\
\hline FD-CTA VRT & 0,40 & 0,48 & 0,07 & 0,25 & 0,54 \\
\hline MD-CTA MPR & 0,54 & 0,31 & 0,05 & 0,45 & 0,63 \\
\hline MD-CTA VRT & 0,83 & 0,37 & 0,06 & 0,72 & 0,94 \\
\hline DSA long & 0,57 & 0,69 & 0,10 & 0,37 & 0,78 \\
\hline DSA middle & 0,51 & 0,66 & 0,10 & 0,31 & 0,70 \\
\hline DSA near & 0,63 & 0,73 & 0,11 & 0,41 & 0,85 \\
\hline DSA rotation MPR & 0,41 & 0,40 & 0,06 & 0,29 & 0,53 \\
\hline$D S A$ rotation $V R T$ & 0,68 & 0,49 & 0,07 & 0,53 & 0,82 \\
\hline$D S A$ rotation long & $-0,05$ & 0,34 & 0,05 & $-0,15$ & 0,05 \\
\hline$D S A$ rotation middle & 0,17 & 0,39 & 0,06 & 0,052 & 0,28 \\
\hline$D S A$ rotation near & 0,12 & 0,25 & 0,04 & 0,05 & 0,20 \\
\hline
\end{tabular}


Tabelle 9: Statistische Analyse der Bland-Altman Plots im Vergleich der DSA_rotation_near mit allen anderen Verfahren

\begin{tabular}{|c|c|c|c|}
\hline $\begin{array}{l}\text { Statistische Da- } \\
\text { ten/Verfahren }\end{array}$ & $\begin{array}{l}\text { Arrhytm. Gemit- } \\
\text { telte Differenz } \\
(95 \% \mathrm{CI})\end{array}$ & $\begin{array}{c}\text { Untere Grenze } \\
\qquad(95 \% \mathrm{CI})\end{array}$ & $\begin{array}{l}\text { Obere Grenze } \\
(95 \% \mathrm{CI})\end{array}$ \\
\hline FD-CTA MPR & $-0,64(-0,91--0,37)$ & $-3,14(-3,60--2,68)$ & $1,86(1,40-2,32)$ \\
\hline FD-CTA VRT & $-0,67(-0,86--0,49)$ & $\begin{array}{l}-2,37 \quad(--2,68-\quad- \\
2,06)\end{array}$ & $1,02(0,71-1,33)$ \\
\hline MD-CTA MPR & $-0,84(-1,05--0,62)$ & $-2,89(-3,27--2,52)$ & $1,22(0,85-1,60)$ \\
\hline MD-CTA VRT & $-1,34(-1,56--1,13)$ & $-3,37(-3,74--2,99)$ & $0,68(0,31-1,05)$ \\
\hline DSA long & $-0,48(-0,63--0,33)$ & $-1,90(-2,16--1,64)$ & $0,94(0,68-1,20)$ \\
\hline DSA middle & $-0,51(-0,67-0,36)$ & $-1,98(-2,25--1,71)$ & $0,95(0,68-1,22)$ \\
\hline$D S A$ near & $-0,58(-0,75--0,41)$ & $-2,15(-2,44--1,86)$ & $0,99(0,70-1,28)$ \\
\hline$D S A$ rotation $M P R$ & $-0,72(-0,98--0,46)$ & $-3,19(-3,64--2,74)$ & $1,75(1,30-2,20)$ \\
\hline$D S A$ rotation $V R T$ & $-0,86(-1,03-0,69)$ & $-2,49(-2,9--2,19)$ & $0,77(0,47-1,07)$ \\
\hline$D S A$ rotation long & $0,89(-0,04-0,21)$ & $-1,13(-1,36--0,91)$ & $1,31(1,09-1,54)$ \\
\hline$D S A$ rotation middle & $0,01(-0,08-0,11)$ & $-0,89(-1,05-0,71)$ & $0,91(0,75-1,08)$ \\
\hline
\end{tabular}


Tabelle 10: Interclass correlation coefficient für alle untersuchten Techniken zum Vergleich der Untersucher-Reliabilität

\begin{tabular}{|c|c|c|}
\hline $\begin{array}{l}\text { Statistische } \\
\text { Daten/Verfahren }\end{array}$ & $\mathrm{ICC}$ & $95 \% \mathrm{CI}$ \\
\hline FD-CTA MPR & 0,8801 & $0,7932-0,9366$ \\
\hline FD-CTA VRT & 0,9518 & $0,9078-0,9759$ \\
\hline MD-CTA MPR & 0,9532 & $0,9065-0,9772$ \\
\hline MD-CTA VRT & 0,9849 & $0,9712-0,9925$ \\
\hline$D S A$ long & 0,9652 & $0,9377-0,9820$ \\
\hline DSA middle & 0,9838 & $0,9705-0,9917$ \\
\hline$D S A$ near & 0,9806 & $0,9648-0,9901$ \\
\hline$D S A$ rotation $M P R$ & 0,9063 & $0,7781-0,9577$ \\
\hline$D S A$ rotation $V R T$ & 0,8638 & $0,7676-0,9275$ \\
\hline$D S A$ rotation long & 0,8638 & $0,7676-0,9275$ \\
\hline$D S A$ rotation middle & 0,9834 & $0,9700-0,9915$ \\
\hline$D S A$ rotation near & 0,9855 & $0,9737-0,9926$ \\
\hline
\end{tabular}




\section{Diskussion}

Nicht zuletzt die ISAT-Studie hat die Relevanz der endovaskulären Aneurysmatherapie verdeutlicht. Die endovaskuläre Therapie hat sich innerhalb der letzten Dekaden als primäre Therapieform etabliert und weiterentwickelt. Entlang der klinischen Entwicklung wurde eine breite Palette von Devices entwickelt, wie z.B. Coils, aber auch Stents, Flow Diverter oder WEB-Devices. Viele von denen sind derzeit Teil der klinischen Praxis (Behme et al. 2015a; Behme et al. 2015b; Gory et al. 2018). Bei all diesen Produkten spielt die exakte Dimensionierung eine erhebliche Rolle. Die Wahl der richtigen Größe des zu verwendenden Device hat einen signifikanten Einfluss auf die Okklusionsraten nach endovaskulärer Versorgung (Behme et al. 2015b; Griessenauer et al. 2016; Hodis et al. 2016). Sowohl in technischer als auch in klinischer Hinsicht sollte die Dimensionierung eine Messgenauigkeit von $\pm 1 \mathrm{~mm}$ haben, da die meisten Devices in 1-mm-Schritten verfügbar sind.

Die Wahl des exaktesten bildgebenden Verfahrens oder, wie in dieser Arbeit gezeigt, die beste Kombination mehrerer bildgebender Verfahren ist damit einer der wichtigsten diagnostischen Schritte im Management von Patienten mit intrakraniellen Aneurysmen. Obwohl die Wahl der Bildmodalität und Rekonstruktionsmethode zunehmend an Bedeutung gewinnt, gibt es bis heute keinen Konsens darüber, wie intrakranielle Aneurysma-Ausmessungen durchgeführt werden sollten (O'meara et al. 2014a; Lauric et al. 2015; Lauric et al. 2018). Nicht zuletzt aufgrund des bestehenden Forschungsdesiderats. Die oben erwähnte Richtgenauigkeit von einem Millimeter ist von klinischer Relevanz. Diese Arbeit setzte sich zum Ziel, die endovaskuläre Versorgung von Aneurysmen und ihr Management zu optimieren, indem sie auf Grundlage einer selbstgeschaffenen Phantomstudie den Stellenwert der zur Verfügung stehenden bildgebenden Verfahren hinsichtlich ihrer Messgenauigkeit bei intrakraniellen Aneurysmen eruieren sollte.

\subsection{Hierarchisierung der Verfahren und Auswertung der Ergebnisse}

Die 2D-DSA gilt als Goldstandard zur Diagnostik intrakranieller Aneurysmen. Auch in dieser Arbeit zeigt die 2D-DSA die besten Messwerte. Die Kombination der 3D-DSA mit der 2D-DSA war allen anderen Untersuchungen überlegen. Die guten Ergebnisse der DSA_rotation_near waren weder von der Größe und Konfiguration der Aneurysmen noch von der Erfahrung des untersuchenden Radiologen abhängig. Bei der 3D-DSA 
wird aus den gewonnenen Datensätzen eine dreidimensionale Darstellung des Aneurysmas erstellt und zwar mit der Option, das Aneurysma aus multiplen Blickwinkeln zu betrachten. Insbesondere intrakraniell wird das Aneurysma häufig durch vor oder hinter ihm verlaufenden, ebenfalls mit KM gefüllten Arterien, überlagert, sodass eine genaue Einsicht nur durch Einstellung eines für jedes Aneurysma neu zu evaluierenden Projektionswinkels mit guter Sicht auf Hals und Dom zu erreichen ist. Der nun ermittelte Projektionswinkel wird als Grundeinstellung für die anschließenden 2D-DAS-Serien genutzt. Wie die Ergebnisse in Kapitel 5 zeigen, sollte zusätzlich der Objekt-zu-Detektorabstand so klein wie möglich gehalten werden, um die geringste Abweichung der Messwerte zur Realgröße zu erreichen. Der Objekt-zu-Detektorabstand ist unterschiedlich, da er abhängig von dem evaluierten Projektionswinkel ist. Bezugnehmend auf die erwähnte klinisch relevante Abweichung von $>1 \mathrm{~mm}$ zeigt die $D S A \_r o t a t i o n \_n e a r$ die besten Ergebnisse mit einer Standardabweichung von 0,07 (0,61) für Aneurysma eins und $0,12(0,25)$ für Aneurysma zwei. Entsprechend der vorbeschriebenen Methodik folgt der Vergleich aller anderer Verfahren mit dem neu definierten Goldstandard. Bei der Betrachtung der erzeugten Bland-Altman-Plots zeigt sich, dass nur noch die DSA_rotation_middle unseren Zielwert von maximal $1 \mathrm{~mm}$ Abweichung erfüllt. Auch wenn alle anderen Verfahren arithmetisch gemittelte Differenzen von $<1 \mathrm{~mm}$ haben, waren ihre oberen und unteren Grenzen signifikant unterschiedlich. Dementsprechend kam es bei vielen Einzelmessungen bei diesen Verfahren zu Abweichungen über einem Millimeter.

Neben den exaktesten Messwerten bietet die DSA_rotation_near, wie alle hier untersuchten invasiven Verfahren, den Vorteil einer direkten Intervention durch das Vorhandensein eines arteriellen Zugangs. Hauptvorteil der 2D-DSA ist die hohe Bildauflösung. Die 2D-DSA ist ein zweidimensionales Verfahren und ist dementsprechend in ihrem Blickwinkel durch die Voreinstellung begrenzt. Dieser Nachteil ist besonders gravierend in der Beurteilung von Pathologien des intrakraniellen Gefäßgeflechtes. Hier kommt es besonders häufig zu Überlagerungen. Aufgrund dieser Tatsache entwickelte sich der Gedanke zur Kombination der 3D-DSA mit einer konventionellen 2D-DSA-Serie durch primäres Explorieren eines optimalen Projektionswinkels für die 2D-DSA. Dadurch konnten die Vorteile zweier Verfahren genutzt werden, um ein ideales Ergebnis bzgl. der Messgenauigkeit zu erreichen. Die Inanspruchnahme des idealen Projektionswinkels durch die 3D-DSA und die hohe Bildauflösung der 2D-DSA erbrachten zusammen die geringste Abweichung zur Referenzgröße. In dieser Arbeit war die 3D-DSA als alleiniges 
Verfahren nicht so exakt in der Ausmessung wie die 2D-DSA-Serien, unabhängig von der Rekonstruktion in MPR oder VRT.

Die MDCTA bedient sich einer Röntgenquelle. Ihr sind mehrere Detektoren in einer gewissen Anordnung gegenübergestellt. Das Strahlenquelle-Detektorkonstrukt rotiert um den Patienten herum und erzeugt so den Datensatz zur Rekonstruktion der Schnittbilder. Zur Verkürzung der Rotationszeit gibt es Systeme mit mehreren Röntgenquellen. Die MDCTA hat sich von Vier-Detektor-Systemen Ende der 90er Jahre zu mittlerweile 320-Detekor-Sytemen entwickelt (Hsiao et al. 2010). Die MDCTA ist ein sensitives Verfahren zur Detektion und Messung intrakranieller Aneurysmen. Sie ist allerdings in Detektion als auch Messgenauigkeit der 2D-DSA sowie der FDCTA unterlegen. Diese Aussage deckt sich mit den Ergebnissen dieser Arbeit sowie den Ergebnissen anderer wissenschaftlicher Arbeiten. Wang et al. verglichen ein 320-Detektorzeilen-CT mit der 2DDSA, indem sie 52 Patienten (insgesamt 54 Aneurysmen) beiden Verfahren unterzogen. Die Ergebnisse kristallisierten zwar die Überlegenheit der 2D-DSA heraus. Allerdings zeigten sie auch die hohe Sensitivität der MDCTA. Bei Aneurysmen, welche größer als 3 mm sind, lag die Sensitivität, Spezifität und Genauigkeit durchweg im mittleren 90\%igen Bereich, leicht unterhalb der 2D-DSA. Allerdings sinkt ihre Sensitivität in dieser Studie bei Aneurysmen, die kleiner als $3 \mathrm{~mm}$ sind auf bis zu 81,8\%. Hier war die DSA deutlich überlegen (Wang et al. 2013). Die MDCT hatte in dieser Arbeit die größten Abweichungen im Vergleich zu den anderen Verfahren. Bei genauerer Betrachtung der Ergebnisse zeigt sich, dass die MDCTA, unabhängig von ihrer Rekonstruktion in MPR oder VRT, die Aneurysmen signifikant überschätzte. Der mittlere Unterschied für die MDCTAMPR betrug 1,15 (1,26) mm für das erste Aneurysma und 0,54 (0,31) für Aneurysma zwei, verglichen mit den bekannten Referenzgrößen.

Die FDCTA war in dieser Arbeit bezogen auf die Messgenauigkeit der 2D-DSA und der 3D-DSA unterlegen. Sie erzielte aber exaktere Messergebnisse als die MDCTA. In dieser Arbeit wurden „leere“, unversorgte Aneurysmen anhand des Phantoms untersucht. Aufgrund der hohen räumlichen Auflösung lassen sich gerade Strukturen mit hohem Kontrast gut in der FDCTA darstellen. Auch wenn für das primäre Management die FDCTA suboptimal ist, so zeigen wissenschaftliche Arbeiten, dass die FDCTA durchaus einen Stellenwert in der Behandlung von Patienten mit Aneurysmen einnehmen kann, nämlich postinterventionell in der Nachsorge endovaskulär therapierter oder postoperierter, durch Clipping versorgter Patienten (Psychogios et al. 2011; Budai et al. 2014). Ott et al. untersuchten dafür 20 Hasen im Tiermodell, in denen experimentell Aneurysmen erzeugt 
wurden. Es zeigte sich, dass die FDCTA eine minimalinvasive Methode zur Ausmessung besonders via Stent versorgten Aneurysmen sein kann und bzgl. ihrer Messgenauigkeit mit der 2D-DSA konkurrieren kann (Ott et al. 2015). Die FDCTA kann dementsprechend für eine spezielle Patientenklientel als Nachsorge-Untersuchung in Betracht gezogen werden. Der Vorteil der FDCTA liegt ähnlich wie der der MDCT in ihrer geringen Invasivität und Schnelligkeit. Dass die FDCTA bei Patienten mit via Stent versorgten Gefäßstenosen eine echte Alternative zur 2D-DSA sein kann, konnten unter anderem Psychogios et al. bereits anhand eines Phantommodells nachweisen (Psychogios et al. 2013).

\subsection{Die Rolle des Untersuchers}

In dieser Studie stellte sich heraus, dass die Erfahrung des Untersuchers keinen signifikanten Unterschied bezogen auf die Messgenauigkeit der verschiedenen Verfahren macht. Die mit dem interclass correlation coefficient angegebene Untersucher-Reliabilität war in jedem Verfahren größer 0,8 und entspricht damit einer fast perfekten Übereinstimmung. In Hinblick auf die Messgenauigkeit spielt dementsprechend die Erfahrung des Radiologen keine große Rolle bezogen auf die hier untersuchten bildgebenden Verfahren. Dieser Rückschluss ist lediglich für die Ausmessung und nicht für das Erstellen des Bildes zulässig. Allen Radiologen wurde nur zur Ausmessung ein nicht bekannter Datensatz vorgelegt. Für die Erstellung des Bildes, vor allem der invasiven Methoden 2D-DSA und 3D-DSA kann diese Arbeit keine Aussagen treffen. Denkbar wären Abweichungen aufgrund von unterschiedlicher Bildqualität, welche durch die Erfahrung in der Durchführung der Angiographie entstehen.

\subsection{Die Gesundheitsrisiken der untersuchten Verfahren}

Betrachtet man die Gesundheitsrisiken der verschiedenen Untersuchungen, so ist die DSA das untersucherabhängigste Diagnostikum der hier untersuchten bildgebenden Verfahren. Das Risiko einer Komplikation, die Strahlenbelastung und die Menge an zu verwendendem Kontrastmittel korrelierten mit der Länge der Untersuchung. Innerhalb der letzten Dekaden hat sich neben der endovaskulären Intervention auch die DSA als Diagnostikum weiterentwickelt, sodass in Zukunft mit einer Abnahme der Komplikationsrate zu rechnen ist. Das Risiko sinkt mit der Erfahrung und damit einhergehend mit der kürzeren Dauer des diagnostischen Eingriffs (Grzyska et al. 1990). Allgemein spart 
man bei einer kürzeren Eingriffsdauer neben der Strahlenbelastung auch Kontrastmittel ein, was wiederum Gesundheitsvorteile für den Patienten bedeutet. Kritisch zu erörtern ist das Risiko, welches durch den invasiven Eingriff entsteht. Bei Patienten, vor allem mit kardiovaskulären Erkrankungen und anderen Komorbiditäten, steigt das peri- und postinterventionelle Risiko. Dabei korreliert das Alter mit dem Risiko (Willinsky et al. 2003). Komplikationen einer DSA umfassen neben den Risiken, welche durch eine arterielle Punktion und die Gabe von Kontrastmittel entstehen, auch die einer arteriellen Dissektion oder dem Auftreten von Thrombembolien. Je nach Ausprägung und Schwere können diese Komplikationen über schwere neurologische Defizite bis hin zum Tode führen.

Die HIPAA-Studie untersuchte die Komplikationsraten bei diagnostisch durchgeführten zerebralen Angiographien. Insgesamt wurden 19826 Patienten evaluiert. In 522 Fällen $(2,63 \%)$ traten neurologische Komplikationen auf, wovon 27 (0,14\%) Apoplexe waren, welche eine irreversible Behinderung für den Patienten zur Folge hatten. Es kam zu zwölf Todesfällen (0,06\%). Ebenfalls zeigte diese Studie eine Risikoerhöhung bei Patienten mit atherosklerotischen Veränderungen der Hirngefäße (Kaufmann et al. 2007). Die Gesamtkomplikationsrate wird von anderen wissenschaftlichen Arbeiten mit 0,30\% angegeben und zeigt, dass man insgesamt von einem geringen Risiko sprechen kann (Fifi et al. 2009). Neben den Risiken schwerer neurologischer Defizite klagen mehr als die Hälfte (55,6\%) aller Patienten über Kopfschmerzen nach durchgeführter zerebraler Angiographie (Kwon et al. 2016). Neben der diagnostischen Exaktheit und Reliabilität ist für die Wahl des geeigneten bildgebenden Verfahrens auch die Patientengefährdung bezüglich der Höhe der ionisierenden Strahlung und des potenziell nephrotoxischen jodhaltigen Kontrastmittels bedeutsam.

Jedes der hier untersuchten Verfahren bedient sich der klassischen Röntgentechnik, also ionisierender Strahlung. Man unterscheidet zwei Arten von Strahlenschäden. Zum einen gibt es deterministische Schäden, also jene, welche ab der Überschreitung eines gewissen Schwellenwerts in jedem Fall auftreten. Ein Beispiel hierfür ist die Strahlenkrankheit. Zum anderen gibt es stochastische Strahlenschäden. Hier gibt es keinen exakten Schwellenwert. Das Auftreten korreliert mit zunehmender Exposition. In diesem Zusammenhang sind besonders Erkrankungen aus dem Bereich der Onkologie zu erwähnen. Die 2D-DSA ist in der interventionellen Radiologie eines der Verfahren mit der höchsten Strahlenbelastung. Dies gilt für den Patienten ebenso wie für das Personal (Steele und Temperton 1993). Die Angiographie zusammen mit den radiologischen Interventionen 
macht zwar in der Bundesrepublik Deutschland derzeit 3\% der verwendeten radiologischen Untersuchungen aus, jedoch sind sie für 17\% der Gesamtstrahlendosis verantwortlich. Nimmt man die CT als Verfahren hinzu, so betragen alle computertomographischen Untersuchungen inklusive Angiographie und interventionellen Eingriffen einen Gesamtdosisanteil von 80\% und das, obwohl sie nur ca. 11\% der radiologischen Untersuchungen ausmachen. Die Anzahl der CT-Untersuchungen in der Bundesrepublik stieg stetig um etwa 40\% zwischen 2007-2014 (Bundesamt für Strahlenschutz Stand 2018).

Wie oben bereits beschrieben, hängt bei Angiographien die Strahlendosis von nicht konstanten Parametern ab. Die Anzahl der benötigten Serien und damit die Durchleuchtungszeit sowie der Strahlengang sind dabei ebenso relevant wie das Vorhandensein und die Verwendung von Schutzmaterialien. So kann die Verwendung von strahlenabweisenden Handschuhen die Strahlendosis für den Untersucher signifikant verringern (Kamusella et al. 2017). Die Strahlendosis der 3D-DSA ist im Vergleich zu gewöhnlichen 2D-DSA-Serien signifikant niedriger. Tsapaki et al. untersuchten die Strahlendosis der 3D-DSA und der 2D-DSA bei 35 Angiographien der ACI. Bei dieser Studie konnten beide Verfahren gleichermaßen die diagnostisch wichtigen Informationen herauskristallisieren. Doch zeigte sich dabei die Differenz in der Strahlendosis (Tsapaki et al. 2008). Katoh et al. bestätigten das Ergebnis der geringeren Strahlendosis der 3D-DSA anhand einer Phantomstudie. Sie zeigten aber, dass in Bezug auf die Bildqualität die konventionelle 2D-DSA der 3D-DSA überlegen ist (Katoh et al. 2011). Durch Erstellen einer 3DDSA kann man aufgrund des dreidimensionalen Bildes einen Projektionswinkel ausmachen und dadurch Bilderserien der 2D-DSA einsparen. Dies ermöglicht durch Kombination beider Verfahren nicht nur eine Reduktion der Strahlendosis, sondern auch die Menge an zu verwendendem Kontrastmittel gegenüber der konventionellen DSA. Hinzukommt, dass eine DSA vor etwaiger Therapie ohnehin obligat ist. Daher ist deren Indikation durch die Notfallsituation, in der sich der Patient befindet, ohnehin gestellt.

\subsection{Wissenschaftliche Einordnung.}

Die Angiographie nimmt heutzutage einen immer größeren Stellenwert in der Diagnostik von Pathologien des Gefäßsystems ein. Zeitgleich zu dieser Entwicklung hat sich die Darstellung intrakranieller Aneurysmen durch die Einführung der DSA und die verschiedenen Rekonstruktionsmöglichkeiten (MPR/VRT) weiterentwickelt. Bislang gibt es wenige Studien, welche sich diesem wichtigen Thema annahmen und die verschiedenen Techniken verglichen. Die Ergebnisse sind widersprüchlich. 
An sechs weißen Neuseeländerkaninchen verglichen Struffert et al. die MDCTA und die 2D-DSA mit der FDCTA. In den Tiermodellen wurden mittels Elastase-Technik Aneurysmen der rechten ACI kreiert und durch die verschiedenen Verfahren ausgemessen. Struffert et al. verglichen die Genauigkeit der Verfahren durch Messung der Aneurysmahöhe, der Breite und der Halsbreite sowie von Gefäßstrukturen wie dem Trunkus brachiocephalicus oder dem Durchmesser der ACI. Es zeigten sich fast identische Ergebnisse der verschiedenen Verfahren. Die FDCTA ist demnach als mögliches Verfahren zur Ausmessung bei Aneurysmen in Tiermodellen anzusehen (Struffert et al. 2010). Eine weitere Forschungsarbeit von Wong et al. verglich die 2D-DSA und die 3D-DSA an 35 Patienten mit aSAB. Sie untersuchten beide Verfahren im Hinblick auf Detektionsfähigkeit, Messgenauigkeit, geometrische Größen und die Abgrenzbarkeit des Aneurysmahalses zum Stammgefäß ebenso wie auch die Strahlendosis, die Durchführungszeit oder den Einsatz von Kontrastmittel während der Untersuchung. Dazu führten sie beide Untersuchungen bei jedem Patienten durch, und zwar erst die 2D-DSA, dann die 3DDSA. Im Punkt Detektion stellte sich eine falsch negative Rate von 6,8 \% (drei von 44) der 2D-DSA gegenüber der 3D-DSA heraus. Bzgl. der Aneurysmagröße konnten Wong et al. keinen signifikanten Unterschied ausmachen. Allerdings wurde in der 3D-DSA der Aneurysmahals relevant größer ausgemessen als in der DSA. Erwartungsgemäß wurde die Abgrenzbarkeit des Aneurysmahalses und die Beziehung zu den umliegenden Strukturen besser in der 3D-DSA dargestellt. Wie in anderen bereits erwähnten Studien zeigte sich auch in dieser Studie, dass die 3D-DSA weniger Kontrastmittel, Strahlendosis und Zeit benötigt als die konventionelle 2D-DSA (Wong et al. 2012b). In einem Untersuchungszeitraum vom zwei Jahren verglichen Wong et al. die Validität zwischen der 2DDSA und der 3D-DSA. Sie untersuchten dafür 20 Patienten mit rupturierten Aneurysmen der ACI. Der gemittelte Unterschied betrug hier weniger als $1 \mathrm{~mm}$ (Wong et al. 2012a). Dies deckt sich mit den Ergebnissen dieser Arbeit. Im Gegensatz zu Wong et al. können wir die 3D-DSA nicht als gleichwertiges Verfahren bzgl. der Messgenauigkeit proklamieren, da die gemessenen Ober- und Untergrenzen in dieser Arbeit signifikante Unterschiede zeigen, die teilweise mehr als einen Millimeter ausmachen und damit nicht dem von uns festgelegten Qualitätsstandard entsprechen. Auch in dieser Arbeit lag die gemittelte Abweichung der 3D-DSA zu den Referenzgrößen unter einem Millimeter.

Der Kernel, auch Faltungskern bzw. Rekonstruktionsalgorhytmus genannt, ist entscheidend für die Bildqualität. Die Wahl des geeigneten Kernels ist meist eine Abwägungsent- 
scheidung zwischen Ort- und Kontrastauflösung. Man unterscheidet harte (hochauflösende) und weiche Kernel. Weiche Kernel eignen sich, wenn man hohe Kontrastauflösung benötigt. In der klinischen Praxis werden diese meist verwendet für NiedrigdosisUntersuchungen bei Kindern oder Schwangeren oder gar für die abdominelle Bildgebung adipöser Patienten. Harte Kernels werden viel in der traumatologischen Diagnostik verwendet, da sie für Bildgebungen mit hohem Bildkontrast geeignet sind, wie es für Skelettuntersuchungen der Fall ist. Sie erhöhen das Bildrauschen und erzeugen Kantenartefakte.

O'meara et al. explorierten die Bedeutung des eingestellten Kernels bezogen auf die Messgenauigkeit intrakranieller Aneurysmen. Bei insgesamt 15 Patienten mit aSAB wurden eine 64-Zeilen-CTA sowie eine Rotationsangiographie durchgeführt. Die MDCTADatensätze wurden mit einem harten und einem weichen Kernel ausgewertet und bezüglich der Halsgröße, Aneurysmabreite und dem Aspect Score verglichen. Die Forschungsgruppe belegt, dass die Wahl des Kernels relevant für die Messgenauigkeit intrakranieller Aneurysmen ist. In der Arbeit von O'meara et al. wurden die verschieden rekonstruierten MDCTA's mit der 3D-DSA als festgelegten Goldstandard verglichen. Demnach überschätzte der weiche Kernel besonders den Aneurysmahals und zwar verglichen mit der Rekonstruktion des harten Kernels und der Rotationsangiographie (O'meara et al. 2014b). Ob nun der Rückschluss, dass die MDCTA mit hartem Kernel ein geeignetes Verfahren bzgl. der Messgenauigkeit intrakranieller Aneurysmen, auch in Bezug auf die oben erwähnte Studie von O'meara et al. zutreffend ist, kann diese Arbeit nicht stützen. Dieses hängt damit zusammen, dass in dieser Arbeit die Rotationsangiographie die Aneurysmen ebenso überschätzte. Wichtig ist allerdings die Erkenntnis, dass die Wahl des Kernels eine signifikante Rolle spielt. Dies gilt nicht nur für die MDCTA und FDCTA, sondern auch für FDCTA und die 3D-DSA, wie die Forschungsarbeit von Lauric et al. belegt (Lauric et al. 2018). Es wurden verschiedene Kernels, betont weiche und normale Kernels, verwendet. Auch in dieser Studie überschätzte die Rotationsangiographie unabhängig von ihrer Rekonstruktion in VRT oder MPR bei weich und normal verwendetem Kernel die Aneurysmagröße. Dies deckt sich mit den Ergebnissen von Lauric et al (Lauric et al. 2018).

Zusätzlich zeigten die Bland-Altman Plots, dass die 3D-DSA unabhängig davon, ob in MPR oder VRT rekonstruiert, das größere Aneurysma stärker überschätzten als das kleinere. Die FDCTA hatte kleinere arithmetisch gemittelte Unterschiede als die MDCTA oder die 3D-DSA. Im direkten Vergleich der FDCTA in MPR und VRT zu der MDCTA 
in MPR und VRT zeigt sich, dass beide kleinere Unterscheide aufwiesen. Dies gilt im Vergleich zum Goldstandard der DSA_rotation_near und den bekannten Aneurysmagrößen. Folglich deckt es sich mit den publizierten Erkenntnissen für intrakranielle Aneurysmen sowie Gefäß- oder Stenosemessungen (Struffert et al. 2010; Psychogios et al. 2013; Zwarzany et al. 2017).

\subsection{Kritische Überlegungen}

Es gibt einige Limitierungen bezüglich der Interpretation und Einordnung der Ergebnisse dieser Studie. Ein Hauptkritikpunkt ist ihr Studiendesign als Phantomstudie. Ein Phantom erzeugt optimale Bedingungen. Der Vorteil eines Phantoms ist der Vergleich zu bekannten Referenzgrößen. Da kein 3D-Druck eine 100\% detailgetreue Nachbildung erzeugt, kann es hier zu einer Variabilität gekommen sein und so die Studienergebnisse beeinflusst haben. Von der klinischen Praxis ebenfalls abweichen kann die Tatsache, dass ein Phantom keine Bewegungsartefakte erzeugt. Der Patient muss es normalerweise schaffen, mehrere Sekunden bis Minuten ruhig dazuliegen. Je nach Compliance, Grunderkrankung, Schmerzen oder Allgemeinzustand ist dies jedoch nicht immer möglich. Auch das Aneurysma-Gefäßmodel ist statisch. Ein pulsierendes Gefäß in vivo kann ebenfalls zu Bewegungsartefakten führen. Eine weitere Einschränkung ist die Erzeugung eines idealen Kontrasts mit einer Dichtedifferenz von 300-400 HU zwischen den Gelkompressen und dem Innenlumen des Aneurysmamodels. Dies realisierten wir durch ein festgelegtes KM-NaCl-Mischungsverhältnis von entweder 1:10 oder 1:2 (s.o.). Eine optimale Kontrastierung ist in der klinischen Praxis bei verschiedenen Patienten, unterschiedlichen Kreislaufzeiten, Größen etc. und weiteren Gründen nicht immer realisierbar. Des Weiteren ist die Platzierung des Modells im Zentrum des Schädels kritisch zu beäugen. Aneurysmen in der Nähe der Schädelbasis sind schwerer zu untersuchen als das in dieser Arbeit zentralplatzierte, wenngleich es ebenfalls in einem humanen Schädel platziert wurde. Relevant ist ebenso die Tatsache, dass alle diese Ergebnisse nur für die hier verwendeten Siemens-Geräte gelten können. Es wäre denkbar, dass andere Hersteller Geräte unterschiedlicher Qualitäten besitzen, sodass an dieser Stelle auch eine Varianz der Ergebnisse existieren könnte. 


\section{Fazit}

Aus dieser Studie geht hervor, dass alle hier untersuchten Techniken insgesamt akkurate Ergebnisse in der Ausmessung intrakranieller Aneurysmen zeigten. Aus klinischer Sicht ist es vor allem für die Planung endovaskulärer Therapien besonders relevant, dass lediglich die 2D-DSA Serien nach perfekt eingestelltem Projektionswinkel durch eine 3DDSA mit geringem oder mittlerem Objekt-zu-Detektorabstand adäquate Messungen unterhalb einer Abweichung von einem Millimeter erzeugen. Dies zeigte diese Studie durch die Bland-Altman-Plots generierten Ober-und Untergrenzen. Zusätzlich zu den exaktesten Messwerten lassen sich Kontrastmittel und Strahlendosis reduzieren, indem weitere DSA-Serien, zur Einstellung einer guten Sicht auf das Aneurysma, unnötig werden.

Da diese Studie Einschränkungen hat und der derzeitige Forschungsstand teils widersprüchliche Ergebnisse liefert, bedarf es weiterer klinischer Forschung zur Beseitigung des bestehenden Forschungsdesiderats in diesem Gebiet. 


\section{Zusammenfassung}

Die SAB ist in der Regel ein schwerer medizinischer Notfall, welcher in den meisten Fällen durch die Ruptur eines intrakraniellen Aneurysmas entsteht. Sie hat meist gravierende Folgen für den Patienten, die nicht selten zu schwerer Behinderung oder Tod führen. Trotz des medizinischen Fortschritts sind die Behandlungsmöglichkeiten nach stattgehabter SAB nach wie vor begrenzt. Die Therapie bedient sich der notfallmäßigen Stillung der Blutung oder der Prävention bei Vorhandensein eines intrakraniellen Aneurysmas. Die endovaskuläre Therapie setzt neben der notfallmäßigen Beendigung einer aSAB, vor allem in der Rupturprävention bei vorhandenem intrakraniellem Aneurysma, an. Mittlerweile sind neben den am häufigsten verwendeten Coils auch andere Medizinprodukte wie Stents etc. auf dem Markt und der klinischen Praxis zu finden. Für die Therapieplanung und Benutzung dieser Devices muss die Größe des Aneurysmas bis auf einen Millimeter genau bekannt sein. Dies ist von klinischer Relevanz, da die Verwendung der exakt passenden Devices signifikant für das etwaige Therapieergebnis ist. Diese Forschungsarbeit setzte sich zum Ziel, eben dieses präinterventionelle Management zu verbessern, indem verschiedene bildgebende Verfahren auf ihre Messgenauigkeit intrakranieller Aneurysmen anhand einer Phantomstudie verglichen wurden. Es wurde die MD-CTA, FD-CTA, die 3D-DSA und die konventionelle 2D-DSA miteinander verglichen. Durch die Kombination der 3D-DSA und der 2D-DSA und der Verwendung zweier Rekonstruktionen, namentlich MPR und VRT, wurden insgesamt 12 verschiedene Bildgebungstechniken untersucht. Das Verfahren, welches den bekannten Referenzgrößen der Aneurysmamodele am nächsten war, ist die DSA_rotation_near. Auch mittelweit eingestellte Objekt-zu-Detektorabstände zeigten ein Ergebnis, das unseren Qualitätsanspruch von $<1 \mathrm{~mm}$ entsprach. Durch das initiale Erstellen einer 3D-DSA kann auf zusätzliche 2D-DSA Serien verzichtet und dadurch Kontrastmittel als auch Strahlendosis reduziert werden. Auch wenn diese Arbeit gewisse Einschränkungen hat, so sollte gemäß dieser Untersuchung die DSA_rotation_near in Zukunft präinterventionell Anwendung finden. 


\section{Literaturverzeichnis}

Ali MS, Starke RM, Jabbour PM, Tjoumakaris SI, Gonzalez LF, Rosenwasser RH, Owens GK, Koch WJ, Greig NH, Dumont AS (2013): TNF- $\alpha$ induces phenotypic modulation in cerebral vascular smooth muscle cells: implications for cerebral aneurysm pathology. J Cereb Blood Flow Metab $\underline{33}$, 1564-1573

Aoki T, Kataoka H, Morimoto M, Nozaki K, Hashimoto N (2007): Macrophage-Derived Matrix Metalloproteinase-2 and -9 Promote the Progression of Cerebral Aneurysms in Rats. Stroke $\underline{38}, 162-169$

Behme D, Weber A, Kowoll A, Berlis A, Burke TH, Weber W (2015a): Low-profile Visualized Intraluminal Support device (LVIS Jr) as a novel tool in the treatment of widenecked intracranial aneurysms: initial experience in 32 cases. J Neurointerv Surg $7,281-$ 285

Behme D, Berlis A, Weber W (2015b): Woven EndoBridge Intrasaccular Flow Disrupter for the Treatment of Ruptured and Unruptured Wide-Neck Cerebral Aneurysms: Report of 55 Cases. AJNR Am J Neuroradiol 36, 1501-1506

Böcher-Schwarz HG, Standhardt H, Perneczky A: Aneurysmata der Hirnarterien. In: Moskopp D, Wassmann H (Hrsg.): Neurochirurgie: Handbuch für die Weiterbildung und interdisziplinäres Nachschlagewerk. 2. Auflage; Schattauer Verlag, Stuttgart 2014, $451-464$

Bor A, Groenestege A, terBrugge K, Agid R, Velthuis B, Rinkel GJ, Wermer MJ (2015): Clinical, Radiological, and Flow-Related Risk Factors for Growth of Untreated, Unruptured Intracranial Aneurysms. Stroke $\underline{46}, 42-48$

Bromberg J, Rinkel G, Algra A, Duyn C, Greebe P, Ramos L, Gijn J van (1995a): Familial subarachnoid hemorrhage: distinctive features and patterns of inheritance. Ann Neurol 38, 929-934

Bromberg J, Rinkel G, Algra A, Limburg M, Gijn J van(1995b): Outcome in familial subarachnoid hemorrhage. Stroke J Cereb Circ 26, 961-963

Bromberg J, Rinkel G, Algra A, Greebe P, Duyn C, Hasan D, Limburg M, Berg H, Wijdicks EF, Gijn J van(1995c): Subarachnoid haemorrhage in first and second degree relatives of patients with subarachnoid haemorrhage. BMJ $\underline{311}$, 288-289

Budai C, Cirillo L, Patruno F, Dall'Olio M, Princiotta C, Leonardi M(2014): Flat Panel Angiography Images in the Post-Operative Follow-Up of Surgically Clipped Intracranial Aneurysms. Neuroradiol J 27, 203-206 
Buhk J-H, Kallenberg K, Mohr A, Dechent P, Knauth M (2009): Evaluation of angiographic computed tomography in the follow-up after endovascular treatment of cerebral aneurysms--a comparative study with DSA and TOF-MRA. Eur Radiol 19, 430-436

Bundesamt für Strahlenschutz (2019): http://www.bfs.de/DE/themen/ion/anwendung-medizin/diagnostik/roentgen/haeufigkeit-exposition.html (zuletzt aufgerufen: 11.02.2019)

Chalouhi N, Ali MS, Starke RM, Jabbour PM, Tjoumakaris SI, Gonzalez LF, Rosenwasser RH, Koch WJ, Dumont AS (2012): Cigarette smoke and inflammation: role in cerebral aneurysm formation and rupture. Mediators Inflamm 2012, 271582

Chalouhi N, Hoh BL, Hasan D (2013): Review of Cerebral Aneurysm Formation, Growth, and Rupture. Stroke 44, 3613-3622

Cossu M, Pau A, Turtas S, Viola C, Viale GL (1993): Subsequent bleeding from ruptured intracranial aneurysms treated by wrapping or coating: a review of the long-term results in 47 cases. Neurosurgery $\underline{32}$, 344-346; discussion 347

DGN (2017a): Deutsche Gesellschaft für Neurologie; Leitlinien der Subarachnoidalblutung Stand 2017. https://www.dgn.org/leitlinien/2318-1l-26-2012-subarachnoidalsblutung-sab (zuletzt aufgerufen 02. Januar 2019)

DGN (2017b): Deutsche Gesellschaft für Neurologie; Leitlinien der unrupturierten intrakranielle Aneurysmen. Stand 2017. https://www.dgn.org/leitlinien/2314-11-25-2012unrupturierte-intrakranielle-aneurysmen (zuletzt aufgerufen 02. Januar 2019)

Dorhout Mees SM, Rinkel GJE, Feigin VL, Algra A, van den Bergh WM, Vermeulen M, van Gijn J (2007): Calcium antagonists for aneurysmal subarachnoid haemorrhage. Cochrane Database Syst Rev CD000277

Fahrig R, Fox AJ, Lownie S, Holdsworth DW (1997): Use of a C-arm system to generate true three-dimensional computed rotational angiograms: preliminary in vitro and in vivo results. AJNR Am J Neuroradiol 18, 1507-1514

Fifi JT, Meyers PM, Lavine SD, Cox V, Silverberg L, Mangla S, Pile-Spellman J (2009): Complications of modern diagnostic cerebral angiography in an academic medical center. J Vasc Interv Radiol 20, 442-447

Fisher CM, Kistler JP, Davis JM (1980): Relation of cerebral vasospasm to subarachnoid hemorrhage visualized by computerized tomographic scanning. Neurosurgery $\underline{6}, 1-9$

Gijn J van, Rinkel G (2001): Subarachnoid haemorrhage: diagnosis, causes and management. Brain 124, 249-278

Gory B, Blanc R, Turjman F, Berge J, Piotin M (2018): The Barrel vascular reconstruction device for endovascular coiling of wide-necked intracranial aneurysms: a multicenter, prospective, post-marketing study. J NeuroInterv Surg 2017-013602 
Greving JP, Wermer MJH, Brown RD, Morita A, Juvela S, Yonekura M, Ishibashi T, Torner JC, Nakayama T, Rinkel G, Algra A (2014): Development of the PHASES score for prediction of risk of rupture of intracranial aneurysms: a pooled analysis of six prospective cohort studies. Lancet Neurol 13, 59-66

Griessenauer CJ, Adeeb N, Foreman PM, Gupta R, Patel AS, Moore J, Abud TG, Thomas AJ, Ogilvy CS, Baccin CE (2016): Impact of Coil Packing Density and Coiling Technique on Occlusion Rates for Aneurysms Treated with Stent-Assisted Coil Embolization. World Neurosurg 94, 157-166

Grzyska U, Freitag J, Zeumer H (1990): Selective cerebral intraarterial DSA. Complication rate and control of risk factors. Neuroradiology $\underline{32}$, 296-299

Guglielmi G, Viñuela F, Dion J, Duckwiler G (1991): Electrothrombosis of saccular aneurysms via endovascular approach. Part 2: Preliminary clinical experience. J Neurosurg $\underline{75}, 8-14$

Hasan D, Chalouhi N, Jabbour P, Hashimoto T (2012): Macrophage imbalance (M1 vs. M2) and upregulation of mast cells in wall of ruptured human cerebral aneurysms: preliminary results. J Neuroinflammation $\underline{9}, 222$

Hodis S, Ding Y-H, Dai D, Lingineni R, Mut F, Cebral J, Kallmes D, Kadirvel R (2016): Relationship between aneurysm occlusion and flow diverting device oversizing in a rabbit model. J Neurointerv Surg $\underline{8}, 94-98$

Hoh BL, Sistrom CL, Firment CS, Fautheree GL, Velat GJ, Whiting JH, Reavey-Cantwell JF, Lewis SB (2007): Bottleneck factor and height-width ratio: association with ruptured aneurysms in patients with multiple cerebral aneurysms. Neurosurgery $\underline{61}, 716-722$; discussion $722-723$

Hop JW, Rinkel G, Algra A, Gijn J van (1997): Case-Fatality Rates and Functional Outcome After Subarachnoid Hemorrhage. A Systematic Review. Stroke 28, 660-664

Hsiao EM, Rybicki FJ, Steigner M (2010): CT Coronary Angiography: 256-Slice and 320Detector Row Scanners. Curr Cardiol Rep 12, 68-75

Hunt WE, Hess RM (1968): Surgical Risk as Related to Time of Intervention in the Repair of Intracranial Aneurysms. J Neurosurg 28, 14-20

Ingall T, Asplund K, Mähönen M, Bonita R, Project for the WM (2000): A Multinational Comparison of Subarachnoid Hemorrhage Epidemiology in the WHO MONICA Stroke Study. Stroke $\underline{31}, 1054-1061$

Ishibashi R, Aoki T, Nishimura M, Hashimoto N, Miyamoto S (2010): Contribution of mast cells to cerebral aneurysm formation. Curr Neurovasc Res $\underline{7}, 113-124$ 
Jamous MA, Nagahiro S, Kitazato KT, Tamura T, Aziz HA, Shono M, Satoh K (2007): Endothelial injury and inflammatory response induced by hemodynamic changes preceding intracranial aneurysm formation: experimental study in rats. J Neurosurg 107, 405-411

Jansen O, Sartor K; Computertomographie. In: Sartor K (Hrsg.): Neuroradiologie. 3., korrigierte Auflage; Thieme, Stuttgart 2006, 8-11

Jayaraman T, Berenstein V, Li X, Mayer J, Silane M, Shin YS, Niimi Y, Kiliç T, Gunel M, Berenstein A (2005): Tumor necrosis factor alpha is a key modulator of inflammation in cerebral aneurysms. Neurosurgery $\underline{57}, 558-564$; discussion 558-564

Kamran M, Nagaraja S, Byrne JV (2010): C-arm flat detector computed tomography: the technique and its applications in interventional neuro-radiology. Neuroradiology $\underline{52}$, 319-327

Kamusella P, Scheer F, Lüdtke CW, Wiggermann P, Wissgott C, Andresen R (2017): Interventional Angiography: Radiation Protection for the Examiner by using Lead-free Gloves. J Clin Diagn Res 11, TC26-TC29

Kashiwazaki D, Kuroda S, Sapporo SAH Study Group (2013): Size ratio can highly predict rupture risk in intracranial small $(<5 \mathrm{~mm})$ aneurysms. Stroke J Cereb Circ $\underline{44}, 2169$ 2173

Katoh M, Opitz A, Minko P, Massmann A, Berlich J, Bücker A (2011): 2D rotational angiography for fast and standardized evaluation of peripheral and visceral artery stenoses. Cardiovasc Intervent Radiol 34, 474-480

Kaufmann TJ, Huston J, Mandrekar JN, Schleck CD, Thielen KR, Kallmes DF (2007): Complications of diagnostic cerebral angiography: evaluation of 19,826 consecutive patients. Radiology 243, 812-819

Kirkpatrick P (2002): subarachnoid haemorrhage and intracranial aneurysms: What Neurologists need to know. J Neurol Neurosurg Psychiatry $\underline{73}$, i28-i33

Kleinloog R, Verweij BH, van der Vlies P, Deelen P, Swertz MA, de Muynck L, Van Damme P, Giuliani F, Regli L, van der Zwan A, et al. (2016): RNA Sequencing Analysis of Intracranial Aneurysm Walls Reveals Involvement of Lysosomes and Immunoglobulins in Rupture. Stroke J Cereb Circ 47, 1286-1293

Korja M, Silventoinen K, McCarron P, Zdravkovic S, Skytthe A, Haapanen A, de Faire U, Pedersen NL, Christensen K, Koskenvuo M, et al. (2010): Genetic epidemiology of spontaneous subarachnoid hemorrhage: Nordic Twin Study. Stroke J Cereb Circ $\underline{41}$, $2458-2462$

Kretzer RM, Coon AL, Tamargo RJ (2010): Walter E. Dandy's contributions to vascular neurosurgery. J Neurosurg 112, 1182-1191 
Kurki MI, Gaál EI, Kettunen J, Lappalainen T, Menelaou A, Anttila V, van 't Hof F, von Und Zu Fraunberg M, Helisalmi S, Hiltunen M, et al. (2014): High risk population isolate reveals low frequency variants predisposing to intracranial aneurysms. PLoS Genet $\underline{10}$, e1004134

Kwon MA, Hong C-K, Joo J-Y, Kim YB, Chung J (2016): Headache After Cerebral Angiography: Frequency, Predisposing Factors, and Predictors of Recovery. J Neuroimaging 26 , 89-94

Lauric A, Heller RS, Schimansky S, Malek AM (2015): Benefit of cone-beam CT angiography in visualizing aneurysm shape and identification of exact rupture site. J Neuroimaging $\underline{25}, 56-61$

Lauric A, Hippelheuser JE, Malek AM (2018): Critical role of angiographic acquisition modality and reconstruction on morphometric and haemodynamic analysis of intracranial aneurysms. J Neurointerv Surg 10, 911-915

Linn F, Voorbij H, Rinkel G, Algra A, van Gijn J (2005): Visual inspection versus spectrophotometry in detecting bilirubin in cerebrospinal fluid. J Neurol Neurosurg Psychiatry $\underline{76}, 1452-1454$

Linn FH, Rinkel GJ, Algra A, van Gijn J (1998): Headache characteristics in subarachnoid haemorrhage and benign thunderclap headache. J Neurol Neurosurg Psychiatry $\underline{65}$, $791-793$

Ljunggren B, Sharma S, Buchfelder M (1993): Intracranial Aneurysms. Neurosurg Q $\underline{3}$, $120-152$

Masuhr KF, Masuhr F, Neumann M: Duale Reihe Neurologie. Georg Thieme Verlag, Stuttgart 2013

Mayer SA, Kreiter KT, Copeland D, Bernardini GL, Bates JE, Peery S, Claassen J, Du YE, Connolly ES (2002): Global and domain-specific cognitive impairment and outcome after subarachnoid hemorrhage. Neurology $\underline{59}, 1750-1758$

McDougall CG, Spetzler RF, Zabramski JM, Partovi S, Hills NK, Nakaji P, Albuquerque FC (2012): The Barrow Ruptured Aneurysm Trial. J Neurosurg 116, 135-144

Meng H, Tutino VM, Xiang J, Siddiqui A (2014): High WSS or low WSS? Complex interactions of hemodynamics with intracranial aneurysm initiation, growth, and rupture: toward a unifying hypothesis. AJNR Am J Neuroradiol $\underline{35}$, 1254-1262

Mistretta CA, Crummy AB, Strother CM (1981): Digital angiography: a perspective. Radiology 139 , 273-276

Molyneux A, Kerr R, Stratton I, Sandercock P, Clarke M, Shrimpton J, Holman R, International Subarachnoid Aneurysm Trial (ISAT) Collaborative Group (2002): Interna- 
tional Subarachnoid Aneurysm Trial (ISAT) of neurosurgical clipping versus endovascular coiling in 2143 patients with ruptured intracranial aneurysms: a randomised trial. Lancet $\underline{360}, 1267-1274$

Molyneux AJ, Kerr RSC, Birks J, Ramzi N, Yarnold J, Sneade M, Rischmiller J, ISAT Collaborators (2009): Risk of recurrent subarachnoid haemorrhage, death, or dependence and standardised mortality ratios after clipping or coiling of an intracranial aneurysm in the International Subarachnoid Aneurysm Trial (ISAT): long-term follow-up. Lancet $\underline{8}, 427-433$

Nakajima N, Nagahiro S, Sano T, Satomi J, Satoh K (2000): Phenotypic modulation of smooth muscle cells in human cerebral aneurysmal walls. Acta Neuropathol (Berl) $\underline{100}$, 475-480

Nieuwkamp DJ, Setz LE, Algra A, Linn FHH, de Rooij NK, Rinkel GJE (2009): Changes in case fatality of aneurysmal subarachnoid haemorrhage over time, according to age, sex, and region: a meta-analysis. Lancet Neurol $\underline{8}$, 635-642

Ott S, Gölitz P, Adamek E, Royalty K, Doerfler A, Struffert T (2015): Flat-detector computed tomography evaluation in an experimental animal aneurysm model after endovascular treatment: A pilot study. Interv Neuroradiol J Peritherapeutic Neuroradiol Surg Proced Relat Neurosci 21, 490-498

O'meara B, Rahal JP, Lauric A, Malek AM (2014a): Benefit of a sharp computed tomography angiography reconstruction kernel for improved characterization of intracranial aneurysms. Neurosurgery 10 Suppl 1, 97-105; discussion 105

Psychogios M-N, Wachter D, Mohr A, Schramm P, Frölich A-M, Jung K, Rohde V, Knauth M (2011): Feasibility of Flat Panel Angiographic CT after Intravenous Contrast Agent Application in the Postoperative Evaluation of Patients with Clipped Aneurysms. Am J Neuroradiol 32, 1956-1962

Psychogios M-N, Schramm P, Amelung N, Pilgram-Pastor S-M, Gröschel K, Wasser K, Frölich AM, Mohr A, Knauth M (2013): Evaluation of noninvasive follow-up methods for the detection of intracranial in-stent restenosis: a phantom study. Invest Radiol $\underline{48}$, 98-103

Raabe A, Schmiedek P, Seifert V, Stolke D (2003): German Society of Neurosurgery Section on Vascular Neurosurgery: Position Statement on the International Subarachnoid Hemorrhage Trial (ISAT). Zentralblatt Für Neurochir 64, 99-103 
Rosengart AJ, Schultheiss KE, Tolentino J, Macdonald RL (2007): Prognostic factors for outcome in patients with aneurysmal subarachnoid hemorrhage. Stroke $\underline{38}, 2315-2321$

Ruigrok YM, Rinkel GJE, Wijmenga C (2005): Genetics of intracranial aneurysms. Lancet Neurol 4, 179-189

Ryu C-W, Kwon O-K, Koh JS, Kim EJ (2010): Analysis of aneurysm rupture in relation to the geometric indices: aspect ratio, volume, and volume-to-neck ratio. Neuroradiology $\underline{53}, 883-889$

Sakaki T, Kohmura E, Kishiguchi T, Yuguchi T, Yamashita T, Hayakawa T (1997): Loss and apoptosis of smooth muscle cells in intracranial aneurysms. Studies with in situ DNA end labeling and antibody against single-stranded DNA. Acta Neurochir 139, 469-474; discussion 474-475

Schievink WI, Torres VE, Piepgras DG, Wiebers DO (1992): Saccular intracranial aneurysms in autosomal dominant polycystic kidney disease. J Am Soc Nephrol $\underline{3}$, 88-95

Schumacher M, Kutluk K, Ott D (1989): Digital rotational radiography in neuroradiology. AJNR Am J Neuroradiol 10, 644-649

Scott RB, Eccles F, Molyneux AJ, Kerr RSC, Rothwell PM, Carpenter K (2010): Improved cognitive outcomes with endovascular coiling of ruptured intracranial aneurysms: neuropsychological outcomes from the International Subarachnoid Aneurysm Trial (ISAT). Stroke J Cereb Circ $\underline{41}, 1743-1747$

Spetzler RF, McDougall CG, Zabramski JM, Albuquerque FC, Hills NK, Russin JJ, Partovi S, Nakaji P, Wallace RC (2015): The Barrow Ruptured Aneurysm Trial: 6-year results. J Neurosurg 123, 609-617

Starke RM, Raper DMS, Ding D, Chalouhi N, Owens GK, Hasan DM, Medel R, Dumont AS (2014a): Tumor necrosis factor- $\alpha$ modulates cerebral aneurysm formation and rupture. Transl Stroke Res $\underline{5}, 269-277$

Starke RM, Chalouhi N, Ding D, Raper DMS, Mckisic MS, Owens GK, Hasan DM, Medel R, Dumont AS (2014b): Vascular smooth muscle cells in cerebral aneurysm pathogenesis. Transl Stroke Res $\underline{5}, 338-346$

Steele HR, Temperton DH (1993): Technical note: patient doses received during digital subtraction angiography. Br J Radiol $\underline{66}$, 452-456

Steiner T, Juvela S, Unterberg A, Jung C, Forsting M, Rinkel G, European Stroke Organization (2013): European Stroke Organization guidelines for the management of intracranial aneurysms and subarachnoid haemorrhage. Cerebrovasc Dis $\underline{35}$, 93-112

Struffert T, Doelken M, Adamek E, Schwarz M, Engelhorn T, Kloska S, Ott S, Doerfler 
A (2010): Flat-detector computed tomography with intravenous contrast material application in experimental aneurysms: comparison with multislice CT and conventional angiography. Acta Radiol 1987 51, 431-437

Sudlow CL, Warlow CP, Collaboration for the ISI (1997): Comparable Studies of the Incidence of Stroke and its Pathological Types Results From an International Collaboration. Stroke $\underline{28}, 491-499$

Teasdale G, Jennett B (1974): Assessment of coma and impaired consciousness. A practical scale. Lancet $\underline{2}, 81-84$

Teasdale G, Drake CG, Hunt W, Kassell N, Sano K, Pertuiset B, De Villiers JC (1988): A universal subarachnoid hemorrhage scale: report of a committee of the World Federation of Neurosurgical Societies. J Neurol Neurosurg Psychiatry $\underline{51}, 1457$

Thron A, Voigt K (1983): Rotational cerebral angiography: procedure and value. AJNR Am J Neuroradiol 4, 289-291

Tsapaki V, Vano E, Muavrikou I, Nueofotistou V, Gallego JJ, Fernandez JM, Santos E, Mendez J (2008): Comparison of patient dose in two-dimensional carotid arteriography and three-dimensional rotational angiography. Cardiovasc Intervent Radiol 31, 477-482

UCAS Japan Investigators, Morita A, Kirino T, Hashi K, Aoki N, Fukuhara S, Hashimoto N, Nakayama T, Sakai M, Teramoto A, et al. (2012): The natural course of unruptured cerebral aneurysms in a Japanese cohort. N Engl J Med $\underline{366}, 2474-2482$

Ujiie H, Tamano Y, Sasaki K, Hori T (2001): Is the aspect ratio a reliable index for predicting the rupture of a saccular aneurysm? Neurosurgery 48 , 495-502; discussion 502 503

van den Berg JS, Limburg M, Hennekam RC (1996): Is Marfan syndrome associated with symptomatic intracranial aneurysms? Stroke J Cereb Circ 27, 10-12

van der Wee N, Rinkel G, Hasan D, Gijn J (1995): Detection of subarachnoid haemorrhage on early CT: is lumbar puncture still needed after a negative scan? J Neurol Neu-

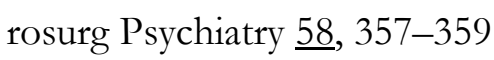

Vermeulen M, van Gijn J (1990): The diagnosis of subarachnoid haemorrhage. J Neurol

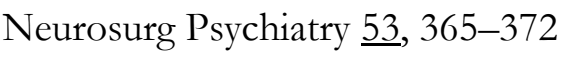

Villablanca JP, Duckwiler GR, Jahan R, Tateshima S, Martin NA, Frazee J, Gonzalez NR, Sayre J, Vinuela FV (2013): Natural history of asymptomatic unruptured cerebral aneurysms evaluated at CT angiography: growth and rupture incidence and correlation with epidemiologic risk factors. Radiology 269, 258-265 
Vlak MH, Algra A, Brandenburg R, Rinkel G (2011): Prevalence of unruptured intracranial aneurysms, with emphasis on sex, age, comorbidity, country, and time period: a systematic review and meta-analysis. Lancet Neurol 10, 626-636

Vlak MH, Rinkel G, Greebe P, Greving JP, Algra A (2013): Lifetime risks for aneurysmal subarachnoid haemorrhage: multivariable risk stratification. J Neurol Neurosurg Psychiatry $\underline{84}, 619-623$

Voigt K, Stoeter P, Petersen D (1975): Rotational cerebral roentgenography. I. Evaluation of the technical procedure and diagnostic application with model studies. Neuroradiology $\underline{10}, 95-100$

Wang H, Li W, He H, Luo L, Chen C, Guo Y (2013): 320-detector row CT angiography for detection and evaluation of intracranial aneurysms: comparison with conventional digital subtraction angiography. Clin Radiol $\underline{68}$, e15-20

Wiebers DO, Whisnant JP, Huston J, Meissner I, Brown RD, Piepgras DG, Forbes GS, Thielen K, Nichols D, O'Fallon WM, et al. (2003): Unruptured intracranial aneurysms: natural history, clinical outcome, and risks of surgical and endovascular treatment. Lancet $\underline{362}, 103-110$

Willinsky RA, Taylor SM, TerBrugge K, Farb RI, Tomlinson G, Montanera W (2003): Neurologic complications of cerebral angiography: prospective analysis of 2,899 procedures and review of the literature. Radiology 227, 522-528

Wong GK, Yu SC, Poon WS (2012a): Radiological measurements of dimensions of acutely ruptured internal carotid artery aneurysm: a comparative study between computed tomographic angiography and digital subtraction angiography. Clin Pract $\underline{2}$, e75

Wong SC, Nawawi O, Ramli N, Abd Kadir KA (2012b): Benefits of 3D rotational DSA compared with 2D DSA in the evaluation of intracranial aneurysm. Acad Radiol 19, $701-$ 707

Xue J, Schmidt SV, Sander J, Draffehn A, Krebs W, Quester I, De Nardo D, Gohel TD, Emde M, Schmidleithner L, et al. (2014): Transcriptome-Based Network Analysis Reveals a Spectrum Model of Human Macrophage Activation. Immunity 40, 274-288

Zwarzany L, Poncyljusz W, Burke TH (2017): Flat detector CT and its applications in the endovascular treatment of wide-necked intracranial aneurysms-A literature review. Eur J Radiol 요, 26-31 


\section{Aus der Arbeit hervorgegangene Veröffentlichung}

Behme D, Amelung N, Khakzad T, Psychogios M.-N: How to Size Intracranial Aneurysms:

A Phantom Study of Invasive and Noninvasive Methods: American Journal of Neuroradiology $2018, \underline{39}$ (12) 2291-2296 


\section{Danksagung}

Wissenschaftliche Arbeiten sind naturgemäß immer das Produkt mehrerer Personen. Ich möchte auf diesem Weg denjenigen danken, die mich bei der Ausarbeitung dieser Arbeit unterstützt haben.

Ein besonderer Dank gilt meinem „Doktorvater“, Herrn PD. Dr. med. Marios Psychogios, der mich auf verschiedenste Weise, von der Themenfindung bis hin zur Veröffentlichung unterstützte. Ohne seine zahlreichen wertvollen Ratschläge und sein Engagement in der Betreuung, wäre diese Arbeit nicht möglich gewesen. Ich verdanke ihm viel. Ebenso danke ich Herrn Dr. med. Daniel Behme, der mich in vielerlei Hinsicht bei der Ausarbeitung der Arbeit, insbesondere der Erstellung der Statistik unterstützte.

Ihnen beiden, zusammen mit Frau Dr. med. Nadine Amelung, möchte ich für die Ausmessung meiner Bilddaten danken. Ohne dieses selbstlose Engagement wäre diese wissenschaftliche Arbeit nicht zustande gekommen.

Ich bedanke mich bei der Firma Phenox für die Herstellung und freundliche Spende der Aneurysmamodelle. 


\section{Widmung}

Diese Arbeit widme ich meinen Eltern, dessen liebevolle Erziehung und uneingeschränkte Unterstützung diese Arbeit und meinen gesamten Lebensweg ermöglichten. 